\title{
A GRAVITY GRADIENT, MOMENTUM-BIASED ATTITUDE CONTROL SYSTEM
} FOR A CUBESAT

\author{
A Thesis \\ presented to \\ the Faculty of California Polytechnic State University, \\ San Luis Obispo
}

\author{
In Partial Fulfillment \\ of the Requirements for the Degree \\ Master of Science in Aerospace Engineering
}

by

Ryan Sellers

March, 2013 
(C) 2013

Ryan Sellers

ALL RIGHTS RESERVED

Page ii 


\section{Committee Membership}

TITLE:

AUTHOR:

DATE SUBMITTED:

COMMITTEE CHAIR:

COMMITTEE MEMBER:

COMMITTEE MEMBER:

COMMITTEE MEMBER:
A Gravity Gradient, Momentum-Biased Attitude Control System for a CubeSat

Ryan Sellers

March, 2013

Dr. Eric Mehiel, Department Chair Aerospace Engineering Department

Dr. Jordi Puig-Suari, Professor Aerospace Engineering Department

Dr. Kira Abercromby, Professor Aerospace Engineering Department

Dr. John Bellardo, Professor Computer Science Department 


\begin{abstract}
A Gravity Gradient, Momentum-Biased Attitude Control System for a CubeSat Ryan Sellers
\end{abstract}

ExoCube is the latest National Science Foundation (NSF) funded space weather CubeSat and is a collaboration between PolySat, Scientific Solutions Inc. (SSI), the University of Wisconsin, NASA Goddard and SRI International. The $3 \mathrm{U}$ will carry a mass spectrometer sensor suite, EXOS, in to low earth orbit (LEO) to measure neutral and ionized particles in the exosphere and thermosphere. Measurements of neutral and ion particles are directly impacted by the angle at which they enter EXOS and which leads to pointing requirements. A combination of a gravity gradient system with a momentum bias wheel is proposed to meet pointing requirements while reducing power requirements and overall system complexity. A MATLAB simulation of dynamic and kinematic behavior of the system in orbit is implemented to guide system design and verify that the pointing requirements will be met. The problem of achieving the required three-axis pointing is broken into four phases: detumbling, initial attitude acquisition, wheel spinup, and attitude maintenance. Ultimately, this configuration for attitude control in a CubeSat could be applied to many future missions with the simulation serving as a design tool for CubeSat developers. 


\section{Acknowledgements}

I want to thank my friends, family, and girlfriend for all of their love and support. I also want to acknowledge all of the work of those in the PolySat lab and CubeSat community upon which my thesis is built on. 


\section{Table of Contents}

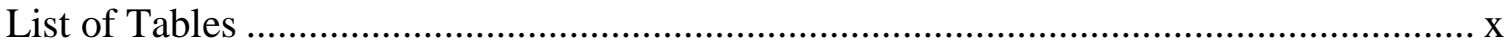

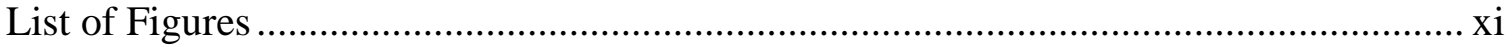

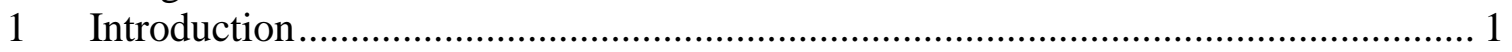

1.1 Scope of Thesis ........................................................................ 1

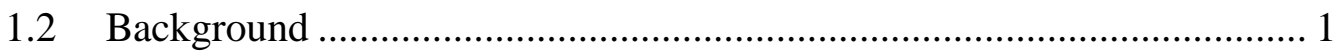

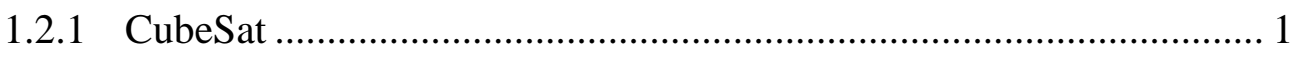

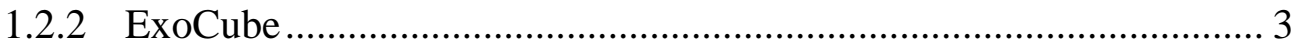

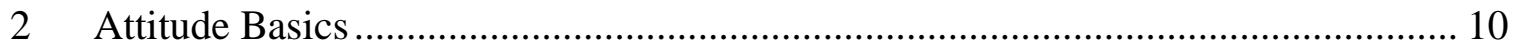

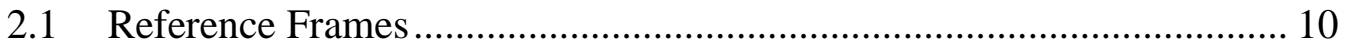

2.1.1 Earth Centered Inertial ........................................................... 11

2.1.2 Earth Centered Earth Fixed ..................................................... 12

2.1.3 Geocentric Latitude, Longitude, Radius ...................................... 13

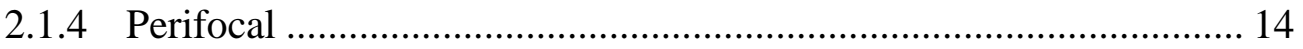

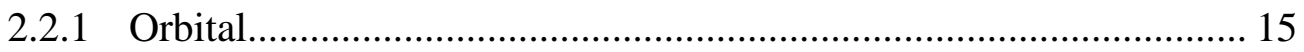

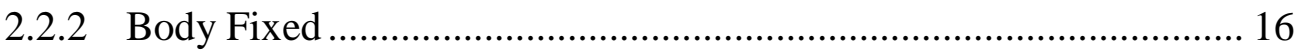

2.3 Attitude Representations ....................................................... 17

2.3.1 Direction Cosine Matrices....................................................... 17

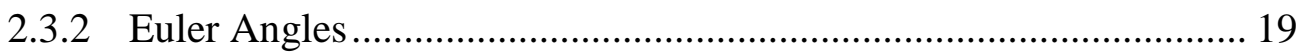

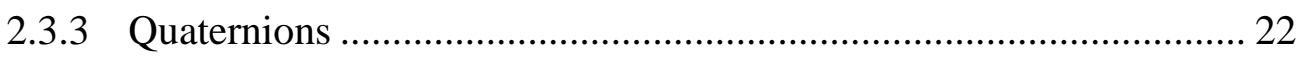

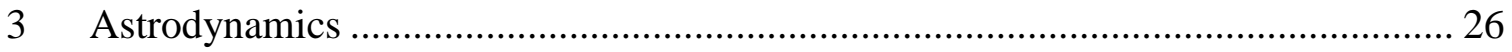

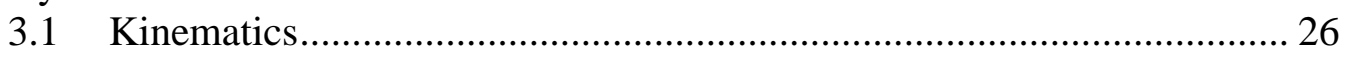

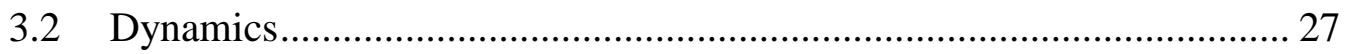

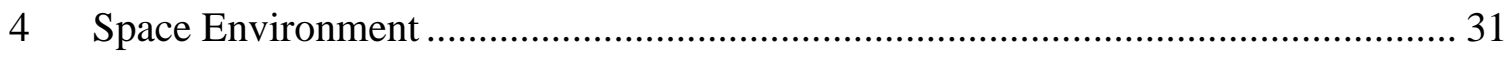

4.1 Earth Magnetic Field Model .............................................................. 31

Page vi 
4.2 Sun Direction........................................................................... 31

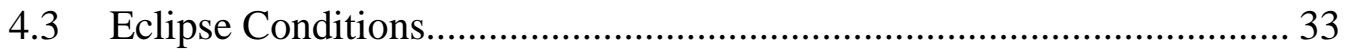

4.4 Atmospheric Model..................................................................... 34

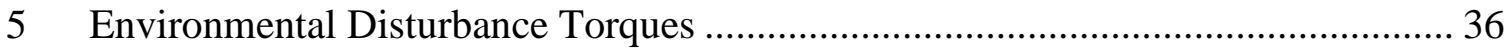

5.1 Gravity Gradient Torque ............................................................ 36

5.1.1 Gravity Gradient Stability .................................................. 37

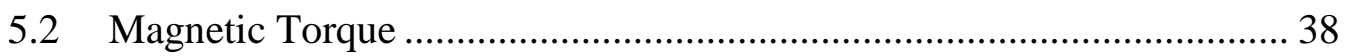

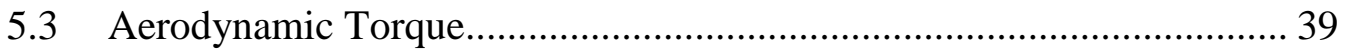

5.4 Solar Radiation Pressure .............................................................. 40

6 Attitude Determination \& Control Architecture ................................................. 42

6.1 Concept of Operations................................................................ 46

6.2 Attitude Determination............................................................... 47

6.3 Attitude Control Hardware .......................................................... 49

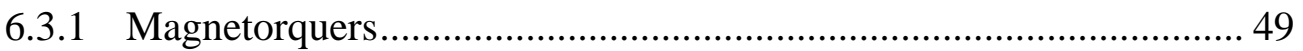

6.3.2 Momentum Wheel................................................................. 54

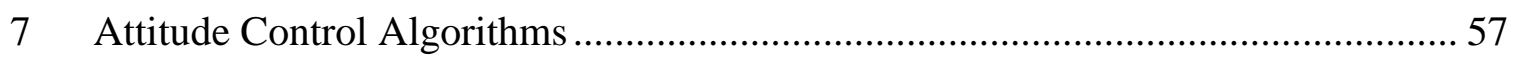

7.1 B-dot Detumbling Algorithm ........................................................ 57

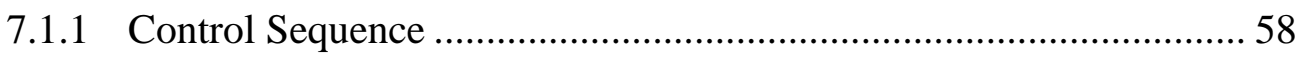

7.2 Three-Axis Control Algorithm.................................................... 59

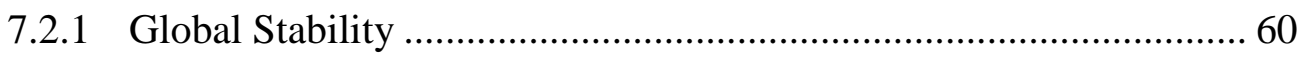

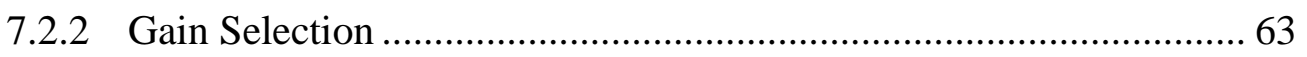

7.2.3 Moment of Inertia Uncertainty .............................................. 64

7.2.4 Pseudo-Reverse Cross Product...................................................... 65

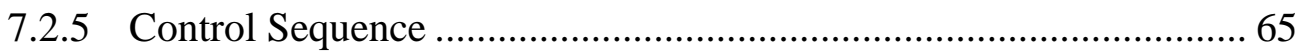

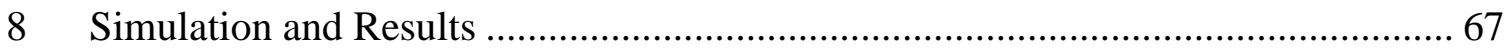

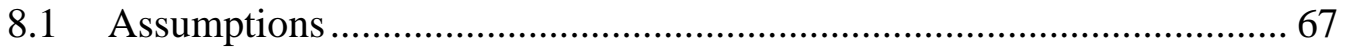




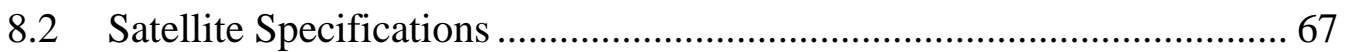

8.3 Guide to B-dot Controller Results Plots................................................... 70

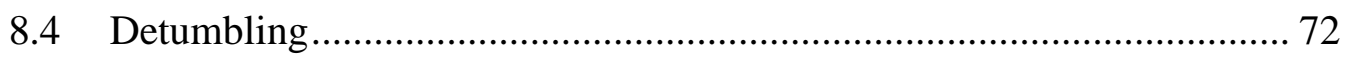

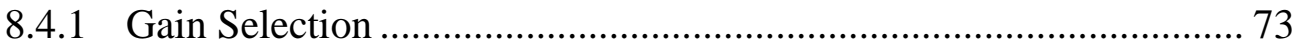

8.4.2 Pulse Time Selection...................................................................... 78

8.4.3 Convergence Criteria Selection....................................................... 81

8.4.4 Minimum Magnetometer Resolution ............................................... 82

8.4.5 Magnetometer Noise Tolerance …………………………………...... 86

8.5 Guide to PD Controller Results Plots....................................................... 87

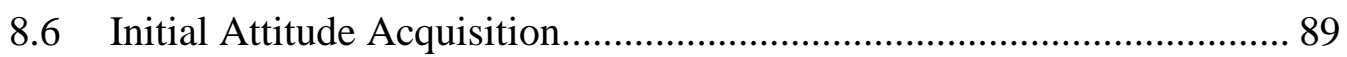

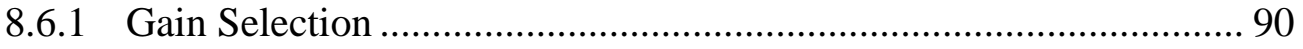

8.6.2 Pulse Time Selection..................................................................... 94

8.6.3 Convergence Criteria........................................................................... 95

8.6.4 Orientation Error Tolerance ………………………………........... 96

8.6.5 Angular Rate Error Tolerance ………………............................. 100

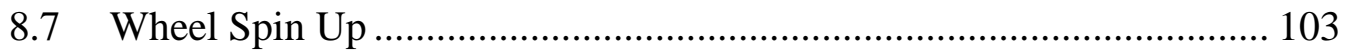

8.7.1 Wheel Spin Up Performance ……………………………………... 106

8.7.2 Wheel Spin Up Performance with Torque Control Noise................ 107

8.8 Attitude Maintenance ............................................................................. 108

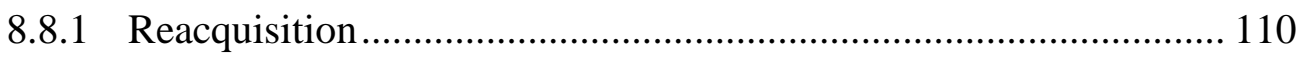

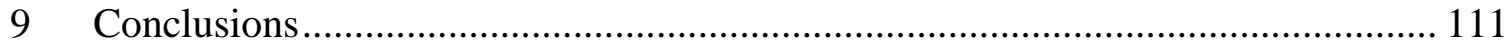

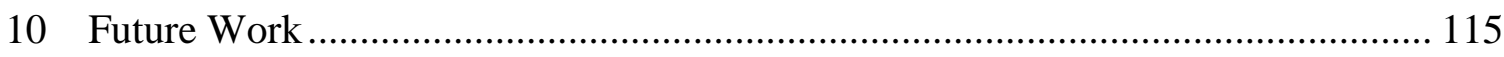

10.1 Hardware in the loop ..................................................................... 115

10.2 Attitude Determination Algorithm ...................................................... 115

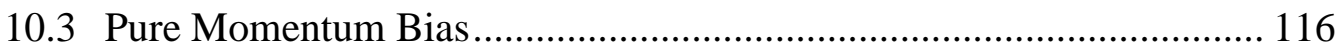




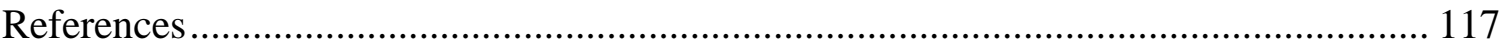

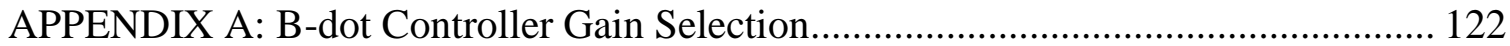

APPENDIX B: PD Controller Line Search for Optimal Gains ................................... 130

Page ix 


\section{List of Tables}

Table 7.1: Summary of worst case environmental torques ...................................... 52

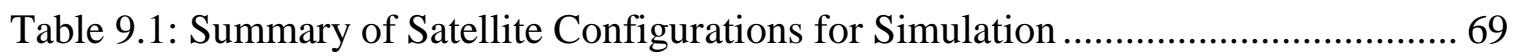

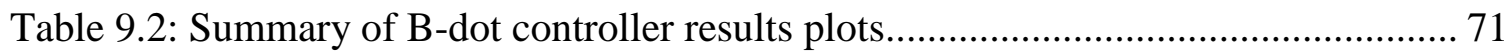

Table 9.3: Summary of PD controller results plots................................................. 88

Table 10.1: Summary of detumbling performance .................................................. 111

Table 10.2: Summary of ideal case initial acquisition performance........................... 112

Table 10.3: Summary of Attitude Determination Algorithm Requirements ................. 113

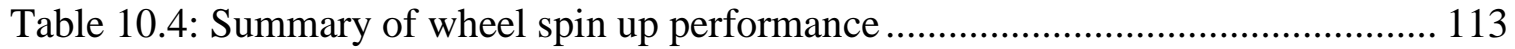




\section{List of Figures}

Figure 1.1: Distribution of Speeds for Gases (Brucat) ……………............................... 4

Figure 1.2: Half Angle Cone of Acceptance Definition ................................................ 7

Figure 2.1: Earth Centered Inertial reference frame …………………....................... 11

Figure 2.2: Earth Centered Earth Fixed reference frame ……........................................ 12

Figure 2.3: Geocentric Latitude, Longitude, Radius reference frame ............................. 13

Figure 2.4: Perifocal reference frame …………………........................................... 14

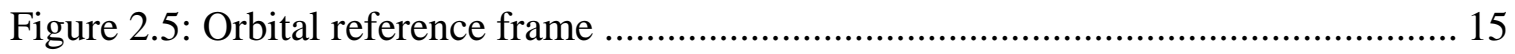

Figure 2.6: Body fixed reference frame ................................................................... 16

Figure 2.7: Direction cosine transformation from xyz to x'y'z' .................................... 18

Figure 2.8: Classical Euler rotation sequence transforming xyz into x'y'z' ...................... 20

Figure 2.9: Yaw, pitch, and roll rotations transforming xyz into x'y'z' .......................... 21

Figure 2.10: Quaternion transformation from xyz to x'y'z' ........................................... 23

Figure 4.1: Components of Earth's Shadow (Eagle) ....................................................... 33

Figure 5.1: Illustration of the gravity gradient effect...................................................... 36

Figure 5.2: The Smelt Parameter Plane (Hall) .............................................................. 38

Figure 6.1: Disturbance Torque Regimes (Turner)....................................................... 44

Figure 6.2: Magnetorquer placement on ExoCube (highlighted in red) ........................... 51

Figure 6.3: Sinclair Interplanetary $10 \mathrm{mNm}$-sec Reaction Wheel ................................... 54

Figure 8.1: Panel representation of spacecraft in deployed configuration......................... 68

Figure 8.2: B-dot performance for selected range of gains .............................................. 73

Figure 8.3: Optimal gain performance of the B-dot controller ........................................ 75

Figure 8.4: Sub-optimal gain performance of the B-dot controller ................................... 76 
Figure 8.5: Simulation depicting local instabilities of B-dot controller due to improper

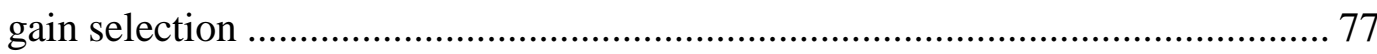

Figure 8.6: B-dot performance for selected range of pulse times ............................... 78

Figure 8.7: Simulation depicting instability of B-dot controller due to increased pulse

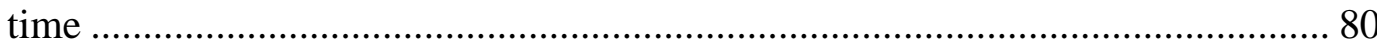

Figure 8.8: B-dot performance for range of selected convergence criteria .................... 81

Figure 8.9: B-dot performance for range of magnetometer resolutions ......................... 82

Figure 8.10: Simulation depicting the impact of magnetometer resolution error on the B-

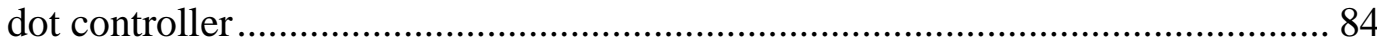

Figure 8.11: Simulation depicting the divergence of the B-dot controller due to magnetometer resolution errors …............................................................ 85

Figure 8.12: B-dot performance for range of magnetometer noise levels ....................... 86

Figure 8.13: Smelt parameter plane with deployed configuration plotted (indicated by red $\mathrm{X})$. 90

Figure 8.14: Optimal gain performance of PD controller for initial acquisition of the target orientation 92

Figure 8.15: Sub-optimal gain performance of PD controller for initial acquisition of the target orientation 93

Figure 8.16: PD controller performance for range of pulse times ................................ 94

Figure 8.17: Spacecraft pointing error for various initial angular rates........................ 96

Figure 8.18: Simulation depicting the impact of quaternion resolution on the PD controller 98

Figure 8.19: Simulation depicting the impact of quaternion noise on the PD controller . 99 Page xii 
Figure 8.20: Simulation depicting the impact of rate resolution on the PD controller... 101 Figure 8.21: Simulation depicting the impact of rate noise on the PD controller .......... 102

Figure 8.22: PD controller performance during wheel spin up ................................ 106

Figure 8.23: PD controller performance during wheel spin up with noise in torque control

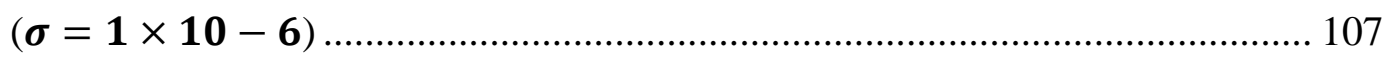

Figure 8.24: PD controller performance for reacquisition of pointing with torque control

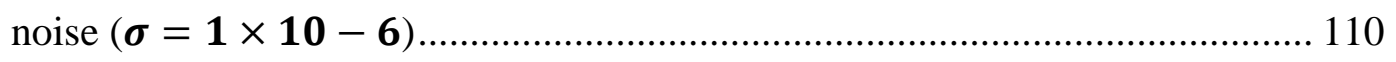




\section{Introduction}

\subsection{Scope of Thesis}

A combination of a gravity gradient system with a momentum bias wheel is proposed to meet pointing requirements while reducing power requirements and overall system complexity. A MATLAB simulation of dynamic and kinematic behavior of the system in orbit is implemented to guide system design and verify that the pointing requirements will be met. The problem is broken into four phases: detumbling, initial attitude acquisition, wheel spin up, and attitude maintenance. Ultimately, this configuration for attitude control in a CubeSat could be applied to many future missions with the simulation serving as a design tool for CubeSat developers.

\subsection{Background}

\subsubsection{CubeSat}

For decades prior to the establishment of the CubeSat standard, universities saw the potential in small satellites as a way to allow students to pursue research projects in space, gain insight into satellite development, and experience the engineering design cycle. Since the budget for most small satellite programs is orders of magnitude smaller than that of industry satellite developers, ridesharing as a secondary payload on a launch proved the most cost effective option. University small satellite programs faced the challenges of the traditional secondary payload in trying to meet the requirements and 
schedule of a specific launch opportunity. Each program faced these challenges individually and often lost launch opportunities due to incompatible requirements, slippage in schedule, or prohibitive costs. These challenges led to longer development cycles which in turn were lengthened more by the loss of institutional knowledge and skill due to the high turnover rate of a university program with students graduating before the completion of a project.

To overcome these challenges, the CubeSat standard was developed through the collaboration of Dr. Jordi Puig-Suari of California Polytechnic State University San Luis Obispo (Cal Poly) and Dr. Bob Twiggs of Stanford University in 1999 with the goal of providing cost-effective access to space for university small satellite programs (CubeSat Design Specification Rev. 12). The standard defines a CubeSat as a $10 \mathrm{~cm}$ cube with a mass less than $1.33 \mathrm{~kg}$ with the purpose of being compatible with a common deployer (CubeSat Design Specification Rev. 12). The CubeSat standard led to the establishment of the student led CubeSat organization at Cal Poly which maintains the standard and developed the Poly Picosatellite Orbital Deployer (P-POD). The P-POD is a flight proven satellite deployment mechanism which can be interfaced with any launch vehicle.

Since the establishment of the standard, CubeSats have evolved from simple student projects into fully capable small satellites which can greatly contribute to military, commercial, and scientific space interests. The standard itself has evolved to include larger CubeSats like the triple unit version known as the $3 \mathrm{U}$. Although originally perceived as too small to be capable of accomplishing meaningful missions, the order of 
magnitude lower cost and acceptance of the risk of commercial off-the-shelf (COTS) parts has made CubeSats a viable solution.

Innovation in making smaller, lower power components driven by the cell phone industry has led to dramatically smaller payloads that previously wouldn't have fit within the CubeSat form factor. Although the payloads are smaller, much of their inherited pointing requirements remain the same, leading CubeSat developers to push the envelope for what is possible in attitude determination and control on a CubeSat. Since CubeSats are constrained in mass and volume which generally translates to power limitations, creative attitude control techniques with low power requirements are needed to meet pointing requirements while maximizing power available to the payload.

\subsubsection{ExoCube}

ExoCube is the latest National Science Foundation (NSF) funded space weather CubeSat and is a collaboration between PolySat, Scientific Solutions Inc. (SSI), the University of Wisconsin, NASA Goddard and SRI International. The 3U will carry a mass spectrometer sensor suite, EXOS, in to low Earth orbit (LEO) to measure neutral and ionized species in the exosphere and thermosphere. The EXOS payload will provide valuable information to the space weather community and is the primary source of pointing requirements for the proposed attitude control system.

Knowledge of upper atmospheric composition is essential to physics-based space weather models. Current knowledge of atmospheric composition uses ground-based incoherent scatter radar (ISR) which carries a higher level of inaccuracy as a result of the 
assumptions used when compared to in-situ mass spectroscopy methods. ExoCube will provide the first in-situ global neutral density data since the era of the Dynamics Explorer 2 (DE-2) satellite (1981-1983), including the first direct measurements of exospheric hydrogen using the mass spectrometer technique.

The EXOS sensor suite features a Static Energy-Angle-Analyzer (SEAA) that focuses incident ion and neutral particles onto a Micro-Channel Plate (MCP) detector. Ions and neutrals impact the MCP at different locations based on their velocity thus the SEAA samples the velocity profile of the various gas species it is exposed to. Figure 1.1 is an example of such a velocity profile showing the Maxwell-Boltzmann speed distribution of gases (It should be noted that not all of these are the species of gases that will be in the exosphere).

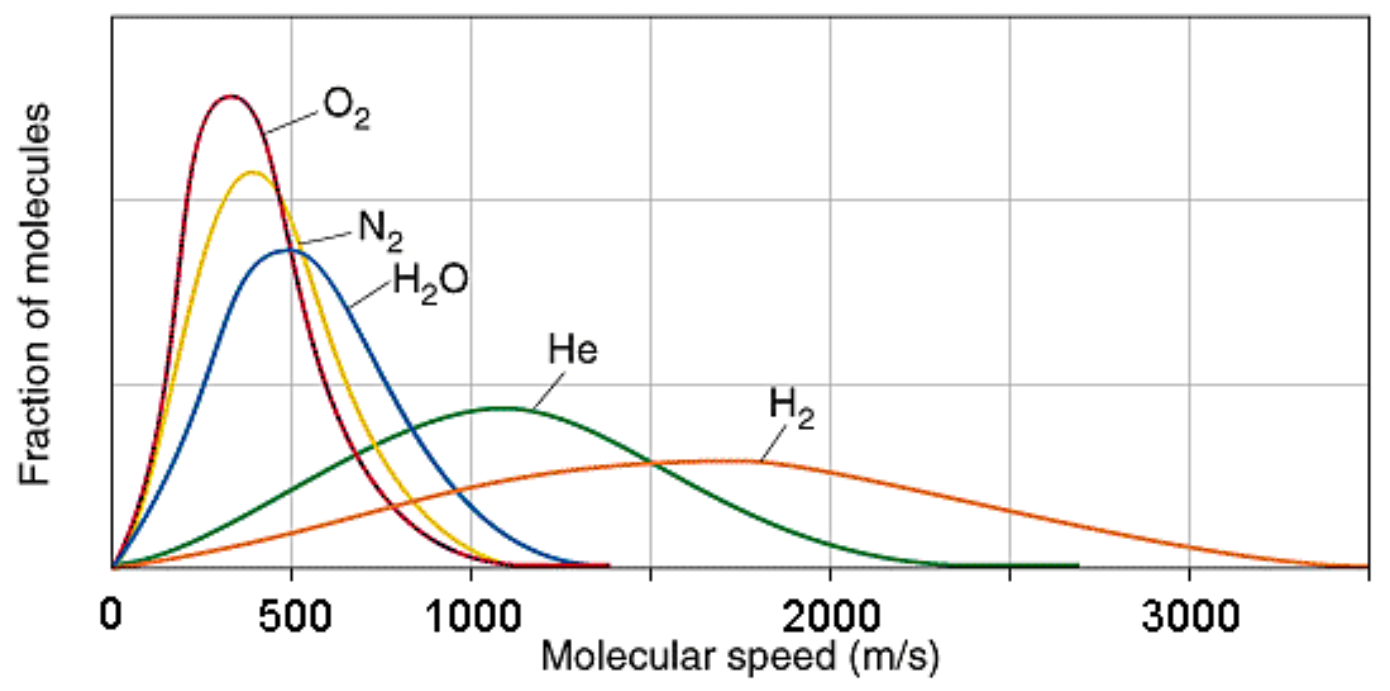

Figure 1.1: Distribution of Speeds for Gases (Brucat) 
Kinetic-molecular theory states that the average kinetic energy of an ideal gas molecule is related to absolute temperature $(T)$ as follows:

$$
K E_{\text {avg }}=\frac{1}{2} m v_{R M S}^{2}=\frac{3}{2} k_{B} T
$$

Where $m$ is the molecular mass in $\mathrm{kg} / \mathrm{mol}, v_{R M S}$ is the root mean square velocity in $\mathrm{m} / \mathrm{s}$, $k_{B}$ is Boltzmann's constant $\left(1.3806503 * 10^{-23} \frac{\mathrm{m}^{2} \mathrm{~kg}}{\mathrm{~s}^{2} \mathrm{~K}}\right)$, and $T$ is the absolute temperature in Kelvin (Brucat). From the kinetic energy formula it can be shown that the root mean square of the velocity is

$$
v_{R M S}=\sqrt{\frac{3 R T}{m}}
$$

Where $R$ is the gas constant and the molar mass $m$ is given in $\mathrm{kg} / \mathrm{mol}$. The standard deviation of the velocity can be defined as (Brucat)

$$
\sigma=\sqrt{\frac{k_{B} T}{m}\left(3-\frac{8}{\pi}\right)}
$$

Using the relation between average kinetic energy and the known absolute temperature of the atmosphere EXOS is exposed to, the peaks in the velocity profile can identify the type and amount of gas species present to produce a density profile. In order to produce an accurate density profile, the velocity profile sample must contain enough data to accurately represent each peak. For EXOS, the ability to capture the peak and $\pm \sigma / 2$ of the velocity distribution is enough information for accurate results. 
In order to accurately capture the velocity profiles of the various gas species in the exosphere, EXOS is designed to be pointed in the ram direction in orbit to collect ions and neutrals. If EXOS points slightly away from the ram direction, the angle at which the ions and neutrals enter the SEAA is effected and thus the velocity profile becomes skewed. The flux of particles entering the aperture of the SEAA becomes limited by the cosine of the difference between the ram direction and the normal vector for the aperture. Provided the offset between the aperture normal vector and the ram direction is a small angle that is known through attitude determination, the loss in flux is minimal and the velocity profile can be calibrated and still provide accurate results. However, if EXOS points far enough off of the ram direction, only the high velocity molecules will be collected thus producing a velocity profile that does not accurately represent all gas species present. In order for the SEAA to have an accurate velocity profile, EXOS cannot point outside a cone around the ram direction defined by the velocity of the slowest molecule measured: atomic oxygen. The half angle of this cone of acceptance can be defined by the triangle made by the spacecraft velocity vector $\left(v_{S / C}\right)$ and the velocity of vector of an atomic oxygen particle traveling in the cross track direction since it is the widest angle possible for a molecule to make with respect to the spacecraft and still be collected. 


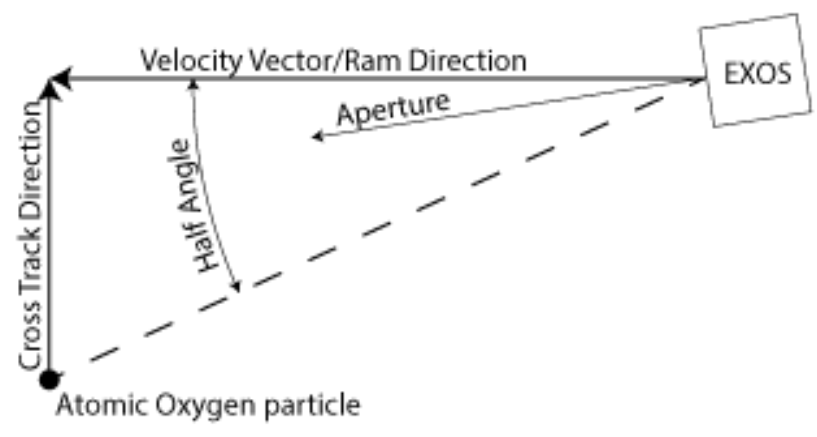

Figure 1.2: Half Angle Cone of Acceptance Definition

Since an accurate representation of atomic oxygen requires capturing a sample of the velocity distribution including the peak and $\pm \sigma / 2$ of the curve, the $v_{[0]}$ component of the cone of acceptance should be

$$
v_{[o]}=v_{R M S}-\frac{\sigma}{2}
$$

Thus the half angle of the acceptance cone can be defined as

$$
\frac{\theta}{2}=\tan ^{-1}\left(\frac{\left(v_{R M S}-\frac{\sigma}{2}\right)}{v_{S / C}}\right)
$$

ExoCube's desired orbit is around $500 \mathrm{~km}$ altitude in the exobase portion of the atmosphere where the median temperature varies with the solar cycle between 1000 and $1600 \mathrm{~K}$. Given this range of expected temperatures and the kinetic energy relationship, the following graph depicts the half angle required to capture the various gas species sampled 


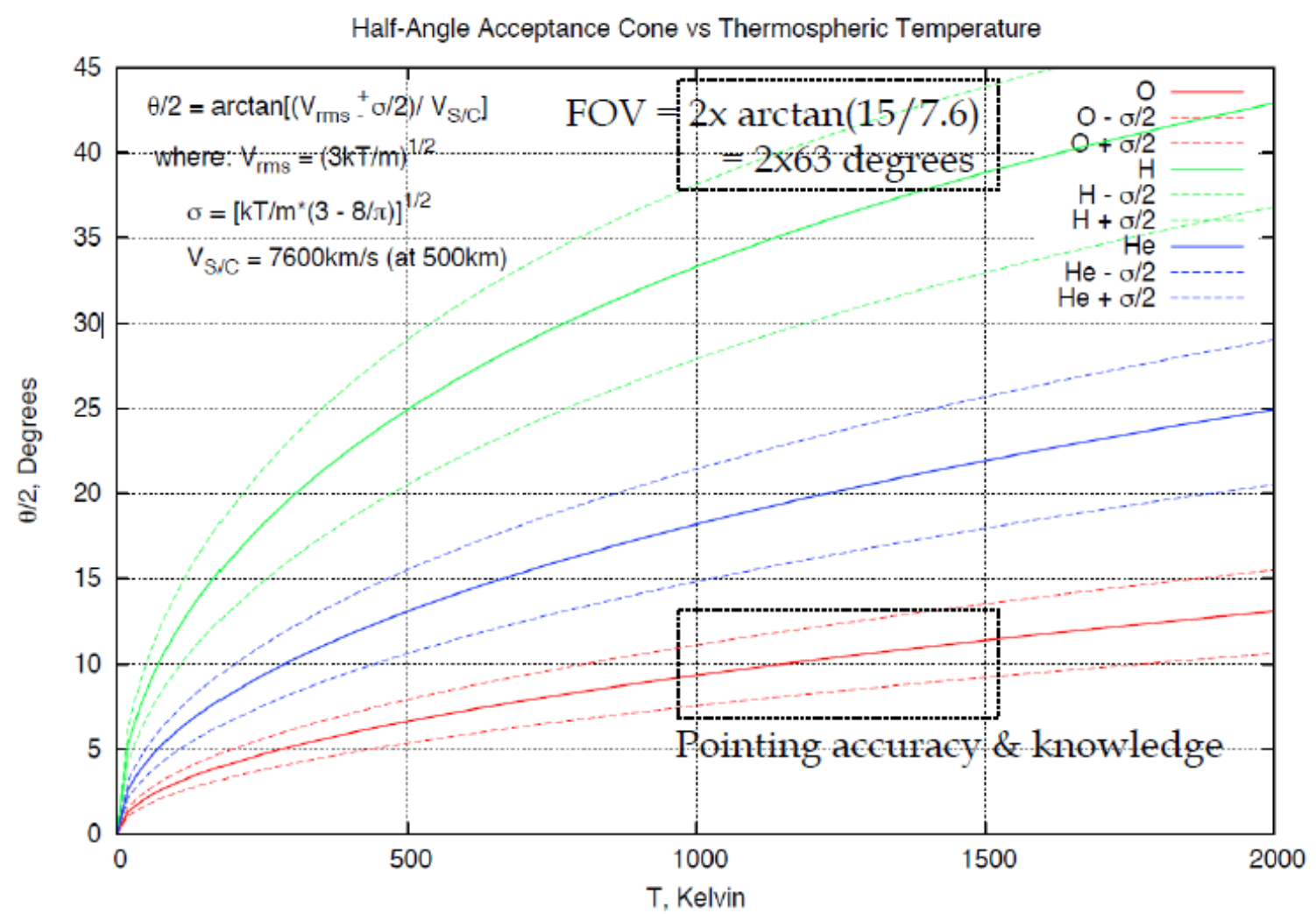

Figure 1.3: Half-Angle Acceptance Cone vs. Thermospheric Temperature (Gardner)

As can be seen in Figure 1.3, the half angle of acceptance for several of the expected gas species are plotted. The solid and dashed lines represent the half angle of acceptance for the range of temperatures for the root-mean-square velocity of each gas species and half of a standard deviation of that velocity respectively. The equations used to generate these lines are shown again on the graph for reference. The black dashed box represents the source of the pointing accuracy requirement. The vertical elements of the black dashed box represent the range of expected thermospheric temperatures while the horizontal elements represent the intersection of the limiting case curves with the range of expected temperatures. The limiting case of the acceptance cone for the range of expected 
temperatures is the line indicating the $O-\sigma / 2$ distribution meaning that EXOS must point within $\pm 8^{\circ}$ of the ram direction to obtain accurate velocity profiles. As development on ExoCube progressed, this requirement was relaxed to $\pm 10^{\circ}$ for the purpose of relaxing the requirements on the attitude determination and control systems at the cost of some loss in accuracy in the oxygen velocity profiles. This loss in accuracy for a single gas species was deemed acceptable in subsequent reviews.

Another critical factor that translates to attitude control requirements is the impact of the slew rate of EXOS within the integration period on the accuracy of the velocity profiles. The SEAA needs to collect neutrals and ions over a period of time on the order of seconds in order to obtain enough information to accurately fit velocity distribution curves to the raw data from individual impacts. Since the angle between the ram direction and the normal vector of the EXOS aperture has the effect of skewing the velocity profile, it follows that a change in angle during the integration period can cause the velocity profile to smear. Although the SEAA has several operational modes, the limiting case comes from when the MCP detector is set to detect the energy of neutral and ion impacts for a range of $25 \mathrm{eV}$ at a resolution of $0.13 \mathrm{eV}$. If the slewing of EXOS causes a change in the impact energy of the ions and neutrals greater than the resolution, the resulting velocity profile will be smeared, introducing an unacceptable level of inaccuracy in the data. The maximum allowable slew rate to prevent smearing of the velocity profile was determined to be $0.1 \mathrm{deg} / \mathrm{s}$. At a slew rate of $0.1 \mathrm{deg} / \mathrm{s}$, the SEAA can collect data for an integration period of up to 10 seconds without appreciable smearing of the velocity profile (Gardner). 


\section{$2 \quad$ Attitude Basics}

The following sections will cover the basics of representing the orientation, or attitude, of a rigid body by first establishing the reference frames used and then by summarizing the methods of representing the relation between two frames.

\subsection{Reference Frames}

The following sections define the reference frames used in the simulation of the attitude dynamics. The frames used follow the conventions found in most sources but it is

important to note that the orbital frame in particular varies between sources and the use of a different convention will impact the results. 


\subsubsection{Earth Centered Inertial}

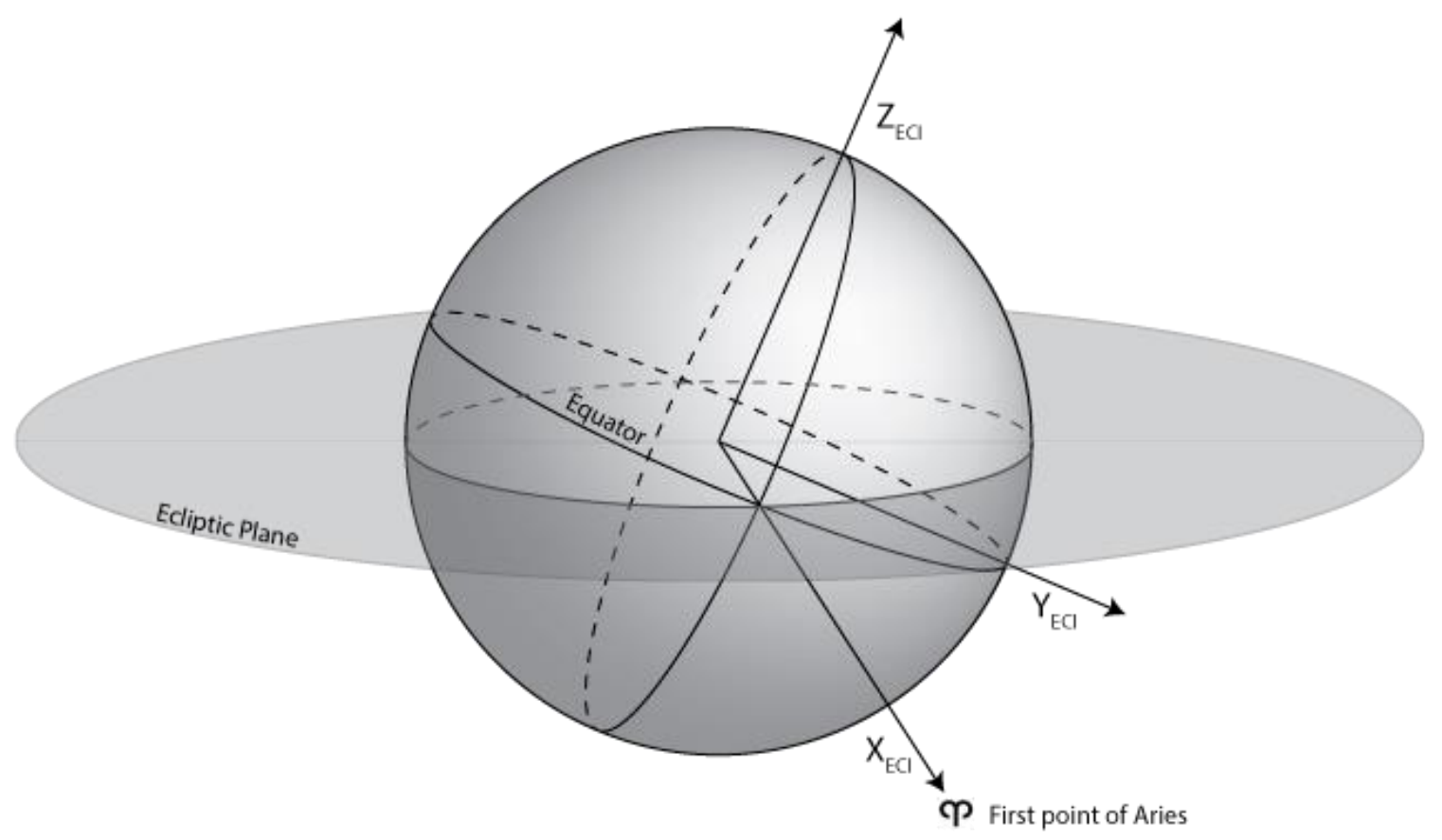

Figure 2.1: Earth Centered Inertial reference frame

The Earth-Centered Inertial (ECI) frame, also known as the geocentric equatorial frame, is a non-rotating right handed Cartesian coordinate system with its origin at the center of the Earth. The fundamental plane $\left(X_{E C I}, Y_{E C I}\right)$ consists of the Earth's equatorial plane with the principal direction $\left(X_{E C I}\right)$ pointed at the first point of Aries $(\mathcal{P})$, the vernal equinox. The right handed coordinate system is completed by $Z_{E C I}$ which is orthogonal to the Earth's equatorial plane and coincides with the Earth's axis of rotation (Curtis). 


\subsubsection{Earth Centered Earth Fixed}

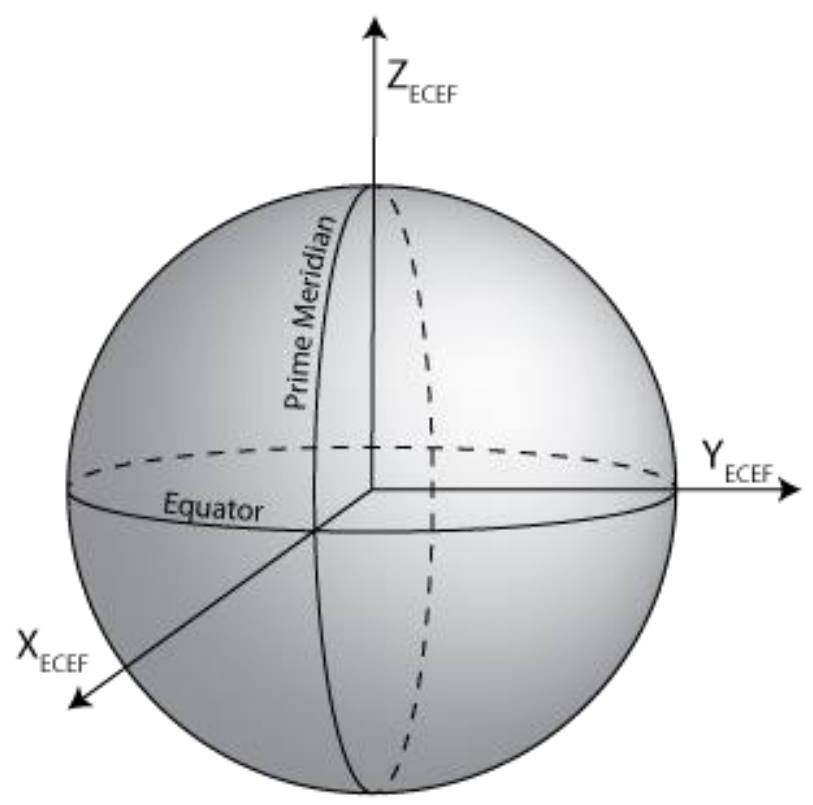

Figure 2.2: Earth Centered Earth Fixed reference frame

The Earth Centered Equatorial Fixed (ECEF) frame rotates at the sidereal period of the Earth with respect to the ECI frame with the origin at the center of the Earth. The fundamental plane $\left(X_{E C E F}, Y_{E C E F}\right)$, consists of the Earth's equatorial plane with the principal direction $\left(X_{E C E F}\right)$ aligned with the intersection of the prime meridian and the equator $\left(0^{\circ}\right.$ Latitude, $0^{\circ}$ Longitude). The right handed coordinate system is completed by $Z_{E C E F}$ which is orthogonal to the Earth's equatorial plane and coincides with the Earth's axis of rotation (Curtis). 


\subsubsection{Geocentric Latitude, Longitude, Radius}

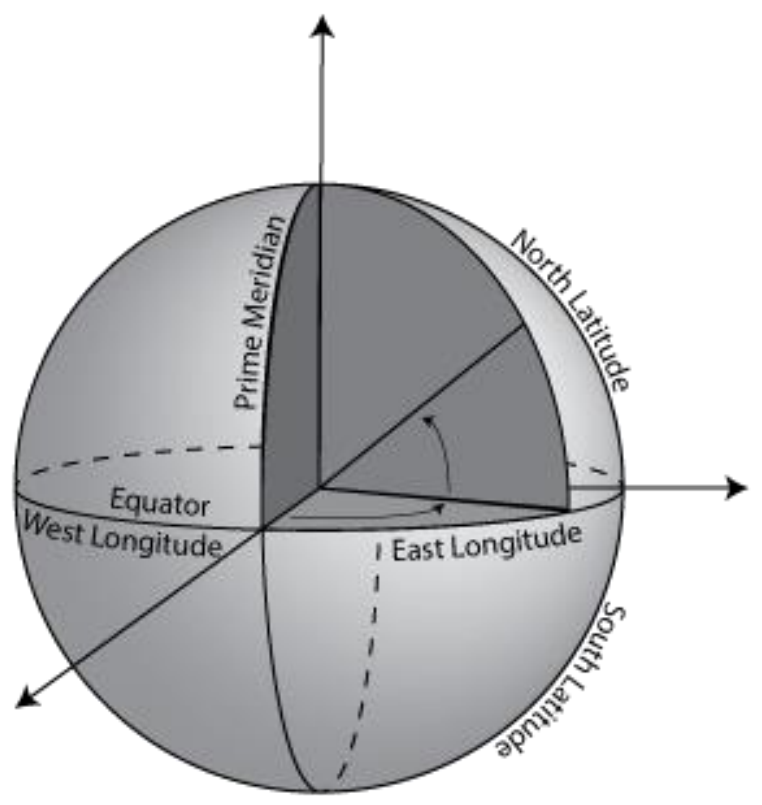

Figure 2.3: Geocentric Latitude, Longitude, Radius reference frame

The Geocentric Latitude, Longitude, and Radius (LLR) frame is the same as the ECEF frame but is expressed in spherical coordinates instead of Cartesian coordinates. Latitude is the angular measurement of North and South of the Equator (North is positive). Longitude is the angular measurement East and West of the Prime Meridian (East is positive). Radius is simply the distance from the center of the Earth to the position of interest (Curtis). 


\subsubsection{Perifocal}

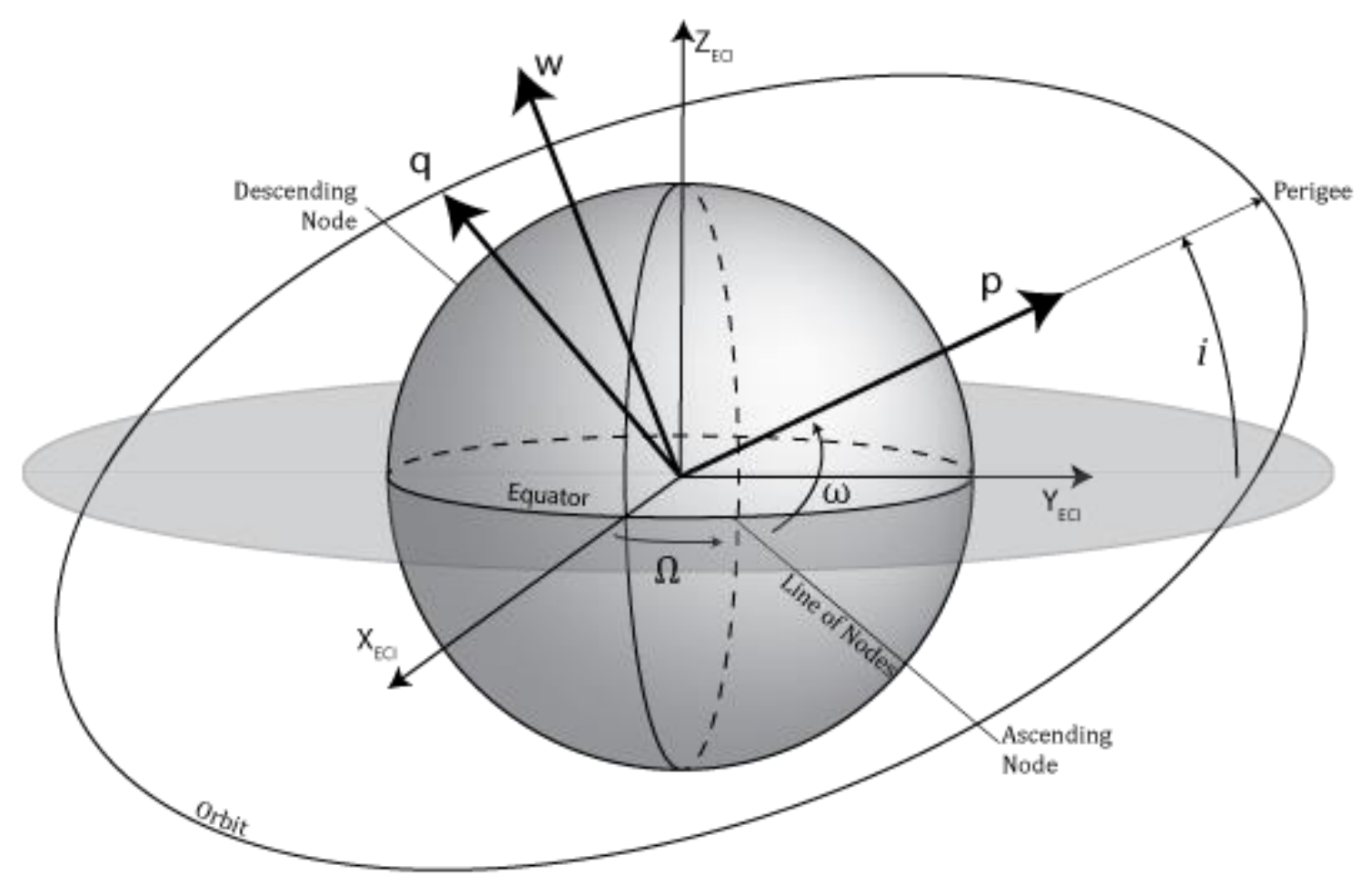

Figure 2.4: Perifocal reference frame

The Perifocal frame is an Earth centered, orbit based, inertial frame. The origin is centered at the focus of the orbit; the center of the Earth. The fundamental plane $(p q)$ is the orbital plane with the principal direction $(p)$ aligned with periapsis and $\mathrm{q}$ at $90^{\circ}$ true anomaly to $p$ axis. The right handed coordinate system is completed by $w$ which is orthogonal to the orbital plane and aligned with the direction of the angular momentum vector (Curtis). 


\subsubsection{Orbital}

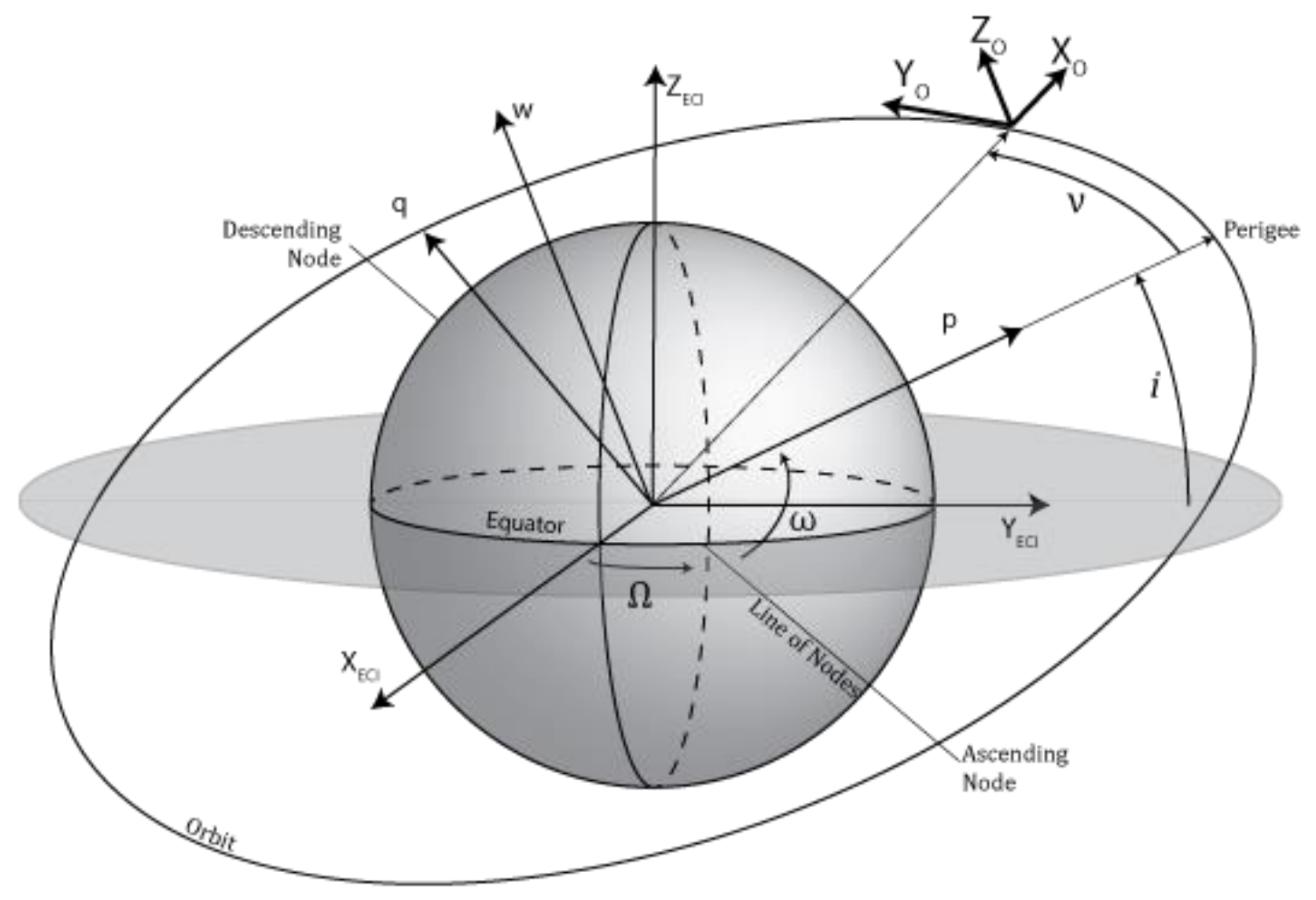

Figure 2.5: Orbital reference frame

The Orbital frame is an Earth centered, orbit based, and rotating frame. The origin is centered at the center of mass of the spacecraft with the $X_{O}$ axis pointing along the position vector, opposite the center of the Earth (nadir). The $Z_{O}$ axis is aligned with the orbital angular momentum vector (cross-track). The right handed coordinate system is completed by $Y_{O}$ which is aligned with the velocity vector (in-track/ram) direction for circular orbits (Kane, Likins and Levinson). 


\subsubsection{Body Fixed}

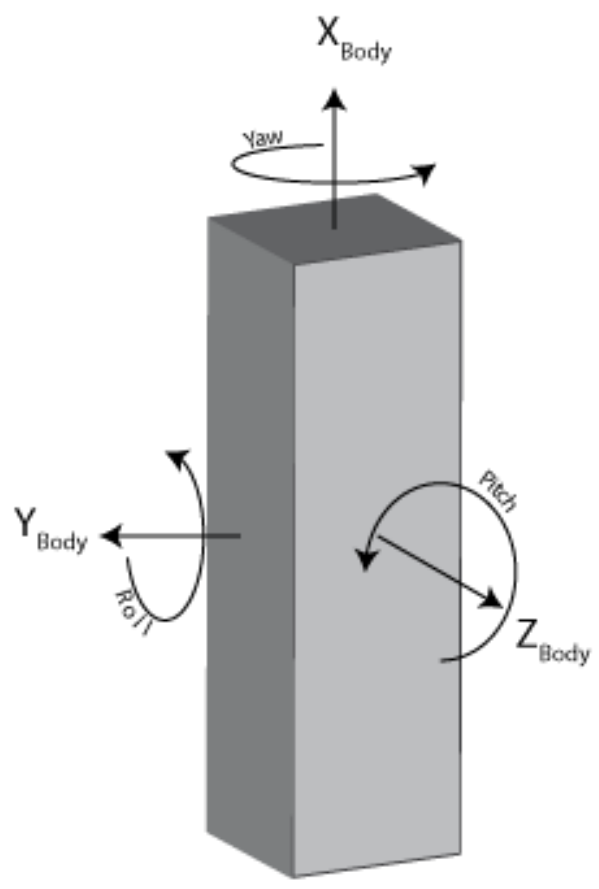

Figure 2.6: Body fixed reference frame

The Body Fixed (Body) frame is aligned geometrically with the spacecraft. The orthogonal set of axes is defined with the origin at the geometric center of the $3 \mathrm{U}$ structure with $\mathrm{X}_{\text {Body }}, \mathrm{Y}_{\text {Body }}$, and $\mathrm{Z}_{\text {Body }}$ axes normal to the sides of the CubeSat as in Figure 2.6. It should be noted that this differs from the coordinate system defined in the CubeSat Design Specification and was done so to match the orbital reference frame and the equations associated with it. Roll, Pitch, and Yaw are defined as rotations about the $\mathrm{X}_{\text {Body }}, \mathrm{Y}_{\text {Body }}$, and $\mathrm{Z}_{\text {Body }}$ axes respectively. 


\subsection{Attitude Representations}

Representing a rigid body in three-dimensional space requires at least three parameters to describe its orientation or attitude with respect to a reference frame. There are several methods to mathematically represent the relationship between two frames. The following section shall serve as an overview of the most common ones: direction cosine matrices (DCM), Euler angles, and quaternions.

Before delving into the most common relationships between frames, it is important to clarify the difference between a vector rotation and a vector transformation. A vector rotation is an operation which changes the orientation of a vector in all coordinate frames. A coordinate transform of a vector is an operation that describes a vector's representation with respect to a different coordinate frame than the original and does not change the orientation of the vector (Groÿekatthöfer and Yoon 4).

\subsubsection{Direction Cosine Matrices}

A direction cosine matrix is a transformation matrix whose elements, the direction cosines, describe the projection of the target coordinate system's basis vectors onto an initial coordinate system's basis vectors. Given an initial frame $A$ and a target frame $B$,

each defined by orthogonal unit basis vectors $\hat{\boldsymbol{\imath}}, \hat{\boldsymbol{j}}$, and $\widehat{\boldsymbol{k}}$, we can express the target frame in terms of the initial frame as follows:

$$
\begin{aligned}
& \hat{\boldsymbol{\imath}}_{\boldsymbol{B}}=Q_{11} \hat{\boldsymbol{\imath}}_{\boldsymbol{A}}+Q_{12} \hat{\boldsymbol{\jmath}}_{\boldsymbol{A}}+Q_{13} \widehat{\boldsymbol{k}}_{\boldsymbol{A}} \\
& \hat{\boldsymbol{\jmath}}_{\boldsymbol{B}}=Q_{21} \widehat{\boldsymbol{\imath}}_{\boldsymbol{A}}+Q_{22} \hat{\boldsymbol{\jmath}}_{\boldsymbol{A}}+Q_{23} \widehat{\boldsymbol{k}}_{\boldsymbol{A}} \\
& \widehat{\boldsymbol{k}}_{\boldsymbol{B}}=Q_{31} \hat{\boldsymbol{\imath}}_{\boldsymbol{A}}+Q_{32} \hat{\boldsymbol{\jmath}}_{\boldsymbol{A}}+Q_{33} \widehat{\boldsymbol{k}}_{\boldsymbol{A}}
\end{aligned}
$$


Where the $Q$ 's are the direction cosines of the basis vectors of $B$. Figure 2.7 illustrates the components of $\widehat{\boldsymbol{k}}_{\boldsymbol{B}}$ which are the projections of $\widehat{\boldsymbol{k}}_{\boldsymbol{B}}$ onto $\hat{\boldsymbol{\imath}}_{\boldsymbol{A}}, \hat{\boldsymbol{J}}_{\boldsymbol{A}}$, and $\widehat{\boldsymbol{k}}_{\boldsymbol{A}}$ (Curtis 216).

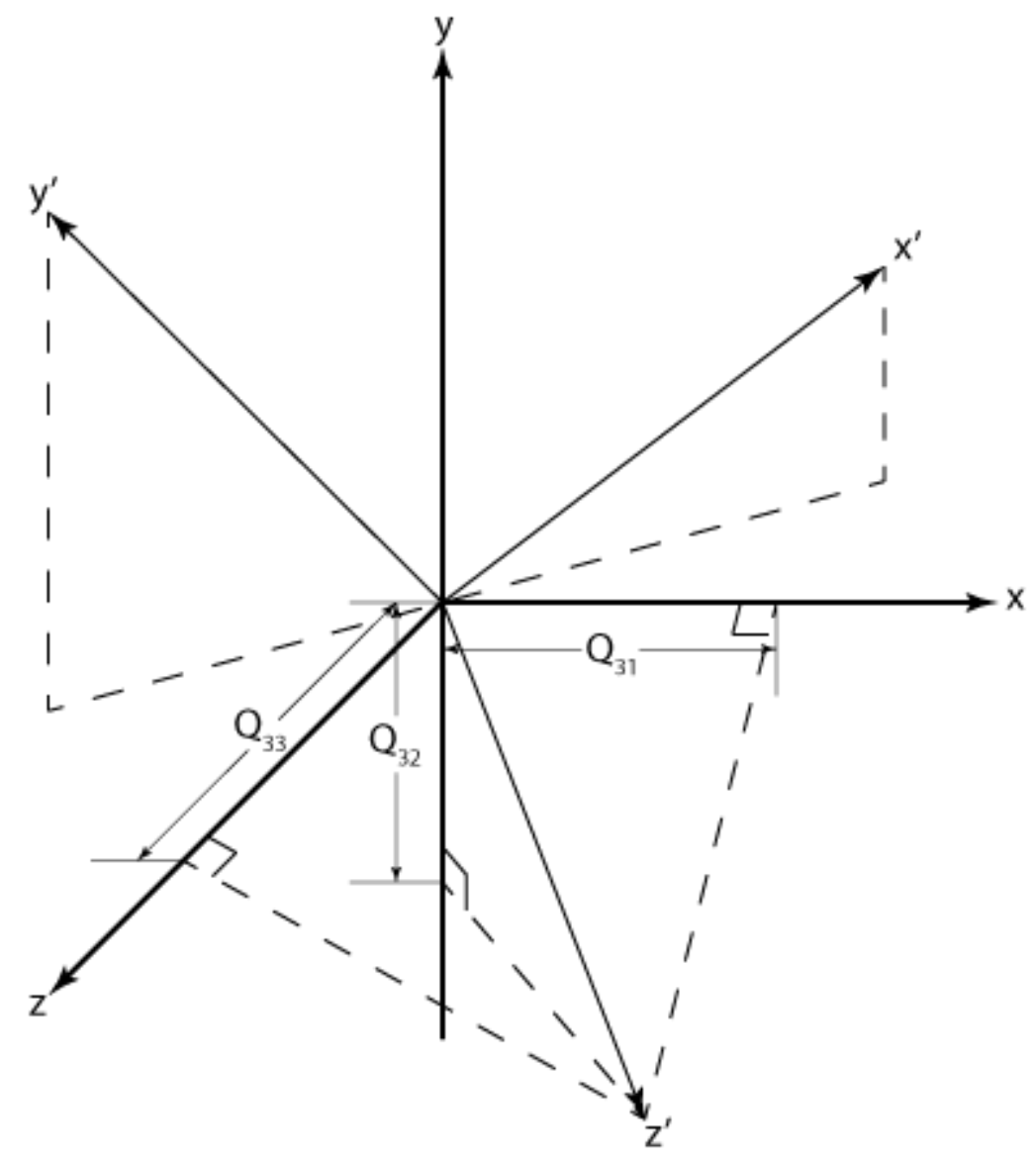

Figure 2.7: Direction cosine transformation from xyz to x'y'z'

The DCM representing the transformation from frame $A$ to frame $B\left(Q_{A B}\right)$ is an orthonormal matrix because the basis vectors of both frames are orthogonal unit vectors. Therefore the transpose of the DCM is the same as the DCM of the inverse transformation:

$$
Q_{A B}^{T}=Q_{A B}^{-1}=Q_{B A}
$$


Transforming a vector in a frame $A$ to frame $B$ can be accomplished by the following equation:

$$
\vec{v}_{B}=Q_{A B} \vec{v}_{A}
$$

Successive transformations through intermediary frames can be accomplished by multiplying successive DCMs as follows:

$$
\vec{v}_{C}=Q_{B C} Q_{A B} \vec{v}_{A}
$$

Note that the order by which the successive transformations are multiplied is right to left starting with the transformation from the initial frame on the right and the transformation to the final frame on the left.

The transformation of a coordinate frame about one of its basis vector through a rotation angle $\theta$ can be described by the following primary transformation matrices:

$$
\begin{aligned}
R_{1}(\theta) & =\left[\begin{array}{ccc}
1 & 0 & 0 \\
0 & \cos (\theta) & \sin (\theta) \\
0 & -\sin (\theta) & \cos (\theta)
\end{array}\right] \\
R_{2}(\theta) & =\left[\begin{array}{ccc}
\cos (\theta) & 0 & -\sin (\theta) \\
0 & 1 & 0 \\
\sin (\theta) & 0 & \cos (\theta)
\end{array}\right] \\
R_{3}(\theta) & =\left[\begin{array}{ccc}
\cos (\theta) & \sin (\theta) & 0 \\
-\sin (\theta) & \cos (\theta) & 0 \\
0 & 0 & 1
\end{array}\right]
\end{aligned}
$$

$R_{l}, R_{2}$, and $R_{3}$ correspond to rotations about the $\hat{\boldsymbol{\imath}}, \hat{\boldsymbol{j}}$, and $\widehat{\boldsymbol{k}}$ axes respectively (Grö̈ekatthöfer and Yoon 4).

\subsubsection{Euler Angles}

The transformation between any two Cartesian coordinate frames can be thought of as a sequence of three transformations using the primary transformation matrices $R_{1}, R_{2}$, and 
$R_{3}$. The sequence of three transformations is called an Euler angle sequence. Since two successive rotations cannot be about the same axis, there are twelve possible Euler angle sequences. One of the sequences that is frequently used in space mechanics is the classical Euler angle sequence:

$$
Q_{\text {Euler }}=R_{3}(\gamma) R_{1}(\beta) R_{3}(\alpha)
$$

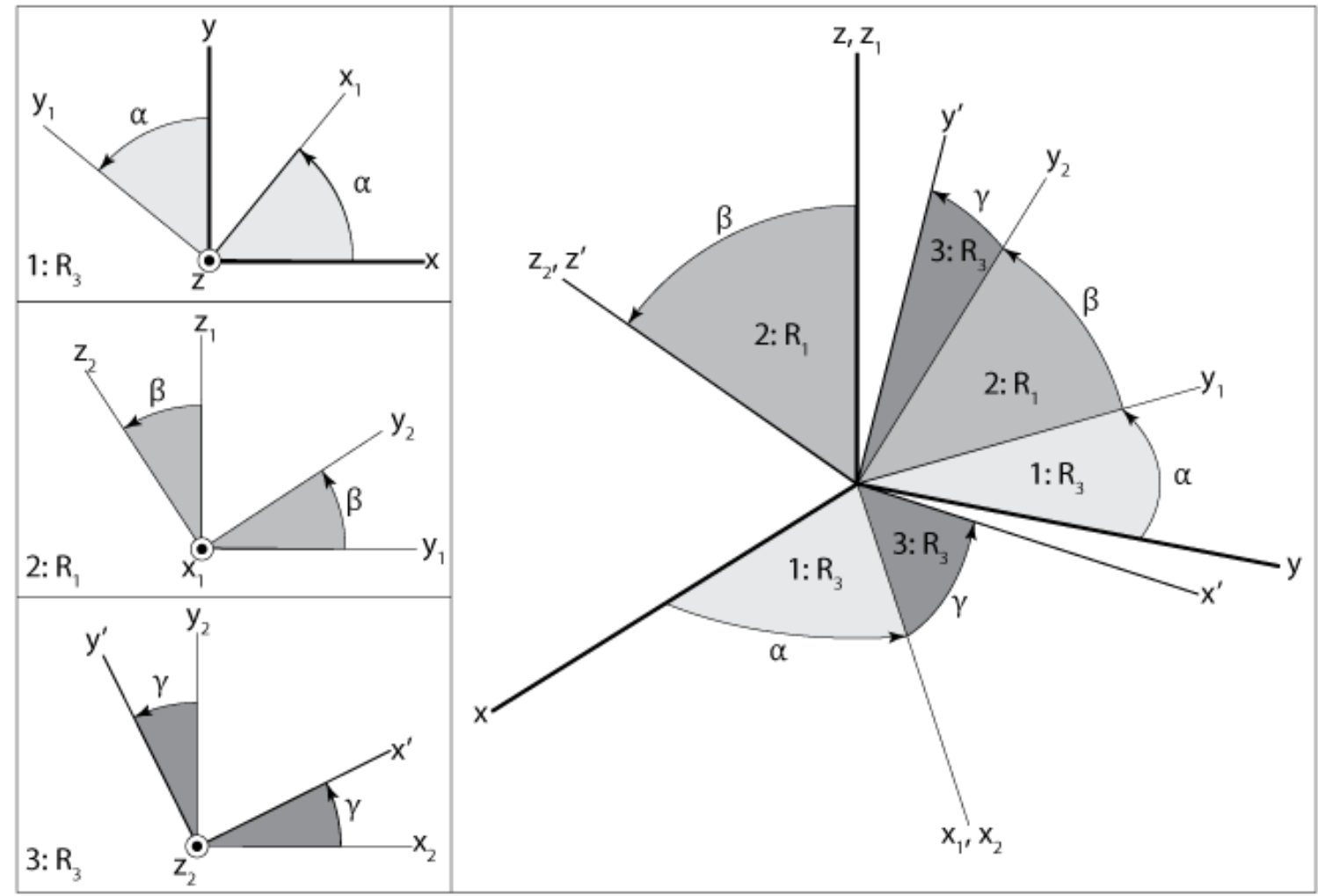

Figure 2.8: Classical Euler rotation sequence transforming $x y z$ into $x^{\prime} y^{\prime} z^{\prime}$

Another common Euler angle sequence in aerospace that is not to be confused with the classical Euler angle sequence is the Yaw, Pitch, Roll (YPR) sequence (Curtis 224):

$$
Q_{Y P R}=R_{1}(\text { Yaw }) R_{2}(\text { Pitch }) R_{3}(\text { Roll })
$$




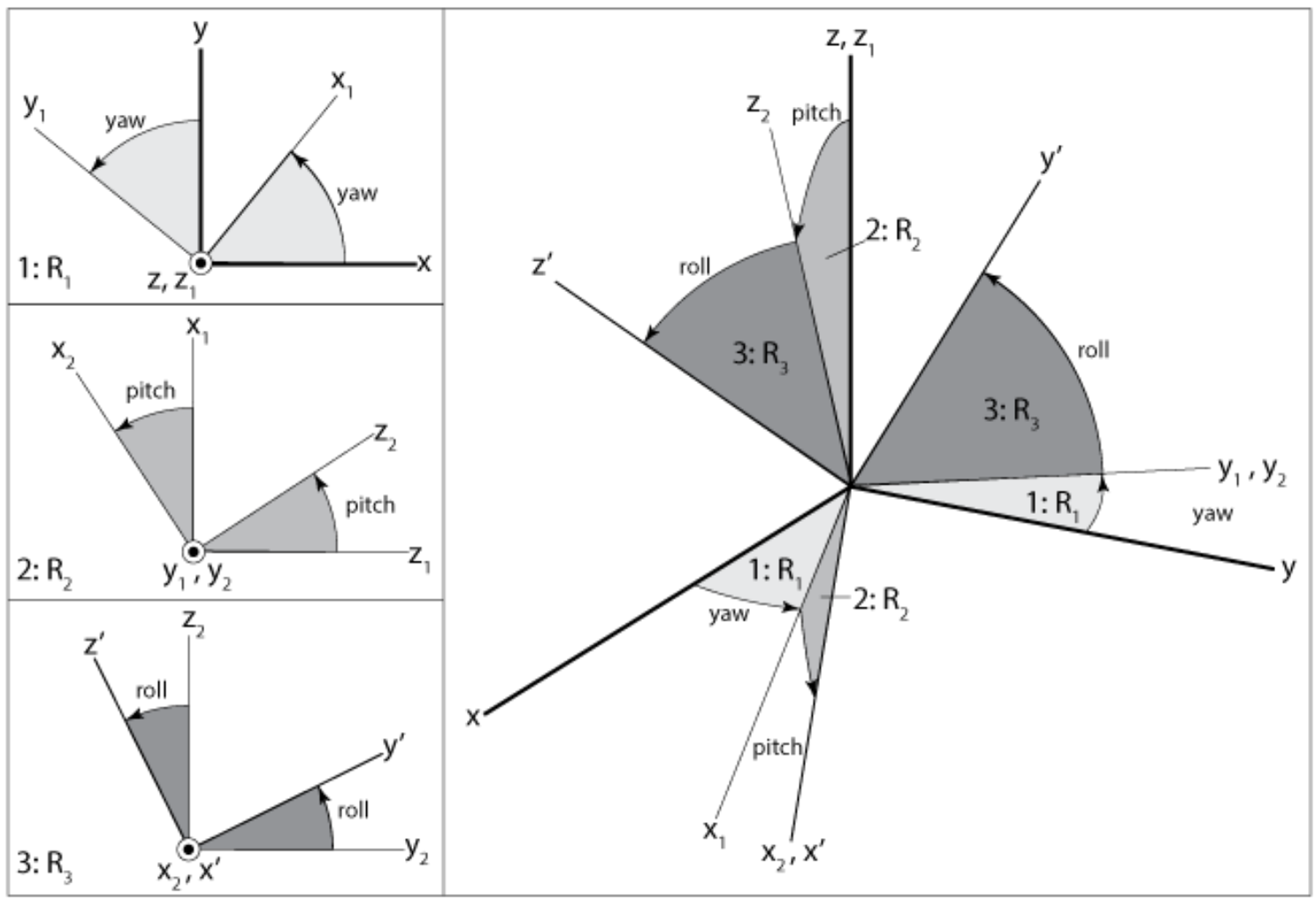

Figure 2.9: Yaw, pitch, and roll rotations transforming $x y z$ into $x^{\prime} y^{\prime} z^{\prime}$

The transformation of a vector via the classical Euler sequence or yaw-pitch-roll sequence can be accomplished by applying the DCM for each sequence in the same manner as the previous section.

Of all the possible different combinations of transformations, Euler angles and yaw-pitchroll representations of attitude are the most commonly used in graphical displays of spacecraft orientation since they are relatively easy to interpret (Groÿekatthöfer and Yoon 5). However, when the nutation angle is 0 degrees in the classical Euler sequence or the pitch angle is 90 degrees in the yaw-pitch-roll sequence, rotation axes align creating a singularity. Thus Euler angle sequence representations are not effective in simulating spacecraft kinematics. 


\subsubsection{Quaternions}

As mentioned in the previous section, Euler angle sequences cannot be relied upon for modeling spacecraft kinematics due to the singularity problems. To avoid singularities that arise from rotating about multiple and possibly aligned rotational axes, we refer to Euler's rotational theorem which states that the relative orientation of two coordinate systems can be described by a unique rotation about a fixed axis through their common origin. This axis of rotation is called the Euler eigenaxis and the angle is referred to as the principal angle. In 1843, Irish mathematician Sir William R. Hamilton (1805-1865) used Euler's rotational theorem to introduce a practical alternative to DCMs: quaternions (Curtis 554).

Quaternions are comprised of four elements. The vector component $(\vec{q})$ is the first three elements and the scalar component is the fourth $\left(q_{4}\right)$. It is common to see the scalar component as either the first or fourth element. The simulations and this paper will have the scalar component as the fourth element as defined below:

$$
q=\left[\begin{array}{c}
\vec{q} \\
q_{4}
\end{array}\right]=\left[\begin{array}{l}
q_{1} \\
q_{2} \\
q_{3} \\
q_{4}
\end{array}\right]
$$

All quaternions for attitude representations are unit quaternions, such that $|q|=1$. Thus we can define them as:

$$
q=\left[\begin{array}{c}
\vec{q} \\
q_{4}
\end{array}\right]=\left[\begin{array}{c}
\hat{u} \sin (\theta) \\
\cos (\theta)
\end{array}\right]
$$


Where $\hat{u}$ is the unit vector along the Euler axis around which the initial frame is rotated into the target frame by the principal angle $\theta$ as shown in Figure 2.10 (Curtis 555).

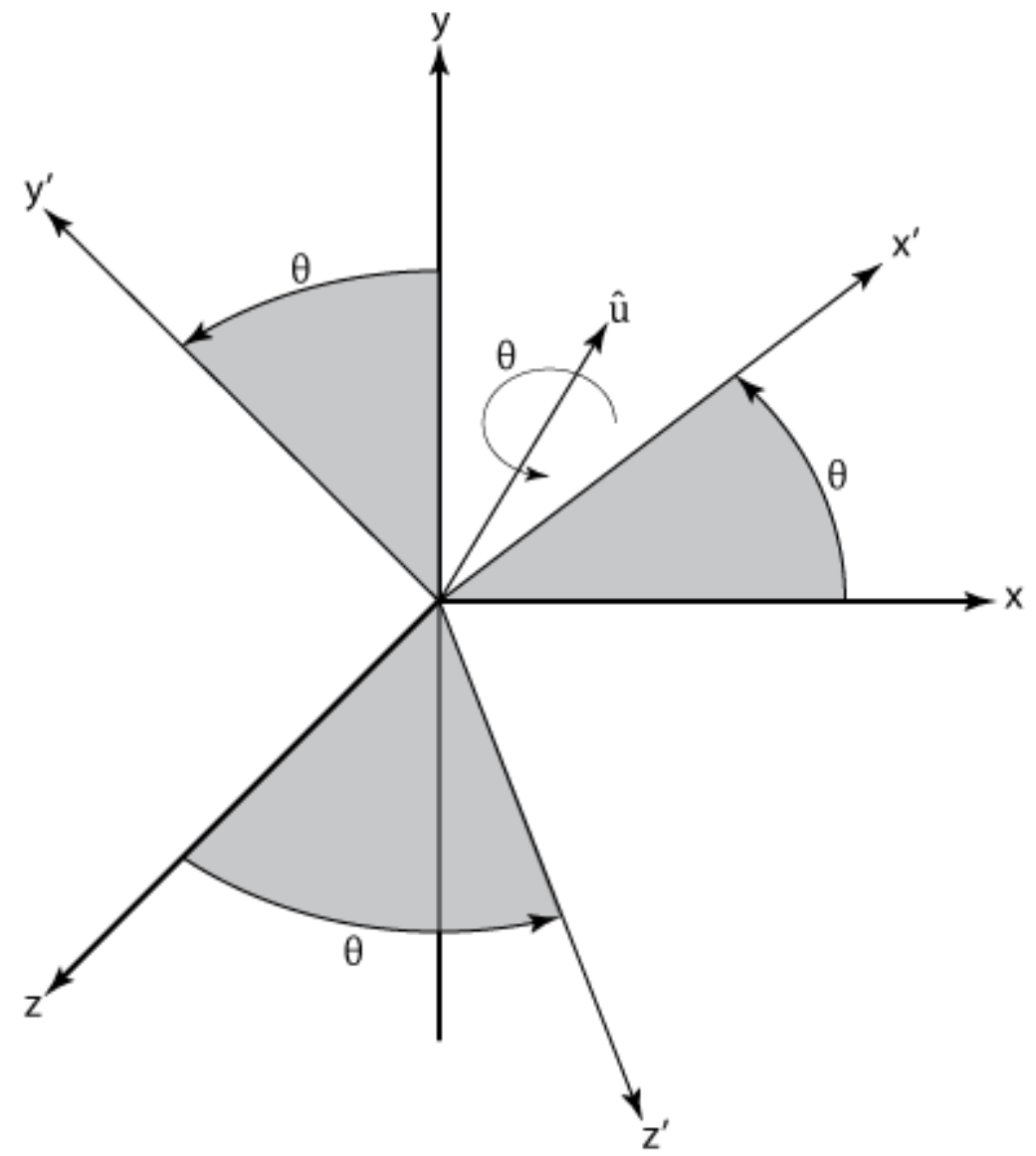

Figure 2.10: Quaternion transformation from xyz to $x^{\prime} y^{\prime} z^{\prime}$

The norm of a quaternion is

$$
|q|=\sqrt{q_{1}^{2}+q_{2}^{2}+q_{3}^{2}+q_{4}^{2}}
$$

The conjugate quaternion has an inverted vector component 


$$
q^{*}=\left[\begin{array}{c}
-\vec{q} \\
q_{4}
\end{array}\right]=\left[\begin{array}{c}
-q_{1} \\
-q_{2} \\
-q_{3} \\
q_{4}
\end{array}\right]
$$

The inverse of a quaternion is its normalized conjugate

$$
q^{-1}=\frac{q^{*}}{|q|}
$$

It should be noted that since all of the quaternions dealt with are unit quaternions, normalization is unnecessary and thus the inverse is the conjugate for unit quaternions.

The product of two quaternions $q_{1}$ and $q_{2}$ is defined by

$$
q_{1} \otimes q_{2}=q=\left[\begin{array}{l}
\vec{v} \\
s
\end{array}\right]=\left[\begin{array}{c}
s_{1} \vec{v}_{2}+s_{2} \vec{v}_{1}+\vec{v}_{1} \times \vec{v}_{2} \\
s_{1} s_{2}-\vec{v}_{1} \cdot \vec{v}_{2}
\end{array}\right]
$$

Where $\vec{v}$ and $s$ are the vector and scalar components of the quaternions respectively.

Vector transformations using quaternions is defined as:

$$
\vec{v}_{B}=q_{A B} \otimes\left[\begin{array}{c}
\vec{v}_{A} \\
0
\end{array}\right] \otimes q_{A B}^{-1}
$$

Successive transformations through intermediary frames can be accomplished by multiplying successive quaternions as follows:

$$
q_{A C}=q_{A B} \otimes q_{B C}
$$

Note that the order by which the successive transformations are multiplied is left to right (the opposite of successive DCMs) starting with the transformation from the initial frame 
on the left and the transformation to the final frame on the right (Groÿekatthöfer and Yoon). 


\section{Astrodynamics}

\subsection{Kinematics}

The angular orientation of a spacecraft can be solved for in quaternions given a known initial quaternion and angular rate with respect to the inertial frame using a numerical differential equation solver on the following governing differential equation:

$$
\begin{gathered}
\dot{\vec{q}}=\frac{1}{2}\left[q_{4} \omega_{b i}-\omega_{b i}^{\times} \vec{q}\right] \\
\dot{q}_{4}=-\frac{1}{2} \omega_{b i}^{T} \vec{q}
\end{gathered}
$$

Where $\omega_{b i}$ is the angular rate of the body with respect to the inertial frame and the quaternion and its derivative also relate the body to the inertial frame (Curtis). The superscript $\times$, as in $\omega_{b i}^{\times}$, represents the skew-symmetric matrix known as the wedge operator which performs the same function as the cross product as defined in the following equation: (Curtis)

$$
\omega^{\times} \vec{q}=\omega \times \vec{q}=\left[\begin{array}{ccc}
0 & -\omega_{3} & \omega_{2} \\
\omega_{3} & 0 & -\omega_{1} \\
-\omega_{2} & \omega_{1} & 0
\end{array}\right] \vec{q}
$$

The wedge operator is used in place of the cross product throughout the simulation as it is more computationally efficient. 


\subsection{Dynamics}

Euler's equation of rotational motion in homogeneous form states that the net moment $\left(M_{\text {net }}\right)$ on a rigid body is equal to the absolute time derivative of its angular momentum $(H)$

$$
M_{\text {net }}=\dot{H}
$$

The absolute time derivative of the angular momentum is the sum of the change in momentum relative to the co-moving frame and the cross product of the angular velocity of the co-moving frame $(\Omega)$ and the momentum. As mentioned in section 3.1, the cross product can be substituted for the wedge operator for the sake of computational efficiency.

$$
\begin{aligned}
\dot{H} & =\dot{H}_{r e l}+\Omega \times H \\
& =\dot{H}_{r e l}+\Omega^{\times} H
\end{aligned}
$$

For a co-moving frame rigidly attached to the body frame,

$$
\Omega=\omega
$$

Given a constant moment of inertia $(I)$, the angular momentum and time derivative become:

$$
\begin{gathered}
H=I \omega \\
\dot{H}_{r e l}=I \dot{\omega}
\end{gathered}
$$


Thus Euler's equation can be rewritten as

$$
M_{n e t}=I \dot{\omega}+\omega^{\times} I \omega
$$

If we add $n$ reaction wheels, the total angular momentum become

$$
H_{a b s}=H_{b o d y}^{c g}+\sum_{i=1}^{n} H_{w h e e l(i)}^{c g}
$$

Where $H_{b o d y}^{c g}$ is the angular momentum of the body without wheels around the center of gravity $(\mathrm{cg})$ of the body and the second term is the sum of the angular momentum of each wheel about the $c g$ of the body. Given constant moments of inertia for the body and the wheels the angular momentum can be rewritten as

$$
H_{a b s}=I_{b o d y}^{c g} \omega+\sum_{i=1}^{n}\left(I_{w h e e l(i)}^{c g(i)} \omega_{w h e e l(i)}^{a b s}+I_{w h e e l(i)}^{c g} \omega\right)
$$

Where $I_{\text {wheel }(i)}^{c g(i)}$ is the moment of inertia of reaction wheel $i$ with respect to its own center of gravity $c g(i)$ while $I_{\text {wheel }(i)}^{c g}$ is the moment of inertia of the same wheel with respect to the $c g$ of the main body. Also, $\omega$ is the angular rate of the body and $\omega_{w h e e l(i)}^{a b s}$ is the angular rate of reaction wheel $i$ with respect to the inertial frame. The two rates are related by

$$
\omega_{\text {wheel }(i)}^{a b s}=\omega_{\text {wheel }(i)}+\omega
$$

Where $\omega_{\text {wheel }(i)}$ is the angular rate of the wheel relative to the body frame. Using this relation and rearranging we obtain 


$$
H_{\text {abs }}=\left(I_{\text {body }}^{c g}+\sum_{i=1}^{n} I_{\text {wheel }(i)}^{c g}\right) \omega+\sum_{i=1}^{n}\left(I_{\text {wheel }(i)}^{c g(i)} \omega_{\text {wheel }(i)}\right)
$$

The sum of the moments of inertia in the first term constitute the total moment of inertia for the spacecraft $\left(I_{\text {total }}\right)$ as follows

$$
I_{\text {total }}=I_{\text {body }}^{c g}+\sum_{i=1}^{n} I_{w h e e l(i)}^{c g}
$$

To simplify notation $I_{\text {wheel }(i)}^{c g(i)}$ can be written as $I_{\text {wheel }(i)}$ and thus the absolute angular momentum can be rewritten as

$$
H_{a b s}=I_{\text {total }} \omega+\sum_{i=1}^{n}\left(I_{\text {wheel }(i)} \omega_{\text {wheel }(i)}\right)
$$

Taking the time derivative we find

$$
\dot{H}_{\text {rel }}=I_{\text {total }} \dot{\omega}+\sum_{i=1}^{n}\left(I_{\text {wheel }(i)} \dot{\omega}_{\text {wheel }(i)}\right)
$$

Where $\dot{\omega}_{\text {wheel }(i)}$ is the relative angular acceleration of wheel $i$. Plugging these back in to Euler's equation for rotational motion gives us

$$
M_{\text {net }}=I_{\text {total }} \dot{\omega}+\sum_{i=1}^{n}\left(I_{\text {wheel }(i)} \dot{\omega}_{\text {wheel }(i)}\right)+\omega^{\times}\left(I_{\text {total }} \omega+\sum_{i=1}^{n}\left(I_{\text {wheel }(i)} \omega_{\text {wheel }(i)}\right)\right)
$$

Rearranging to obtain the differential equation for the numerical solver for the simulation we obtain (Curtis 617) 


$$
\dot{\omega}=I_{\text {total }}^{-1}\left(M_{\text {net }}-\sum_{i=1}^{n}\left(I_{\text {wheel }(i)} \dot{\omega}_{\text {wheel }(i)}\right)-\omega^{\times}\left(I_{\text {total }} \omega+\sum_{i=1}^{n}\left(I_{\text {wheel }(i)} \omega_{\text {wheel }(i)}\right)\right)\right)
$$




\section{Space Environment}

\subsection{Earth Magnetic Field Model}

An accurate model of the Earth's magnetic field is necessary to model the input to magnetometers as well as model magnetic torques. The International Geomagnetic Reference Field (IGRF) was introduced by the International Association of Geomagnetism and Aeronomy (IAGA) in 1968 as a standard spherical harmonic representation of the Earth's main field that is updated every five years and is available at http://www.ngdc.noaa.gov/IAGA/vmod/. The model has a precision of 0.1 nano-Teslas per year (IGRF Guide). This simulation uses the MATLAB code magfd.m created by Maurice A. Tivey of the Woods Hole Oceanographic Institution to compute the Earth's magnetic field components using the IGRF-10 model.

\subsection{Sun Direction}

The direction of the Sun in ECI coordinates are necessary for simulating the input to sun sensors and solar radiation pressure. The Astronomical Almanac provides formulas for computing the Sun's position to within a precision of $0.01^{\circ}$. The following formulas outline the method provided by the Astronomical Almanac:

The number of days since J2000.0 ( $n$ ) is calculated from the Julian Date (JD)

$$
n=J D-2451545.0
$$

The mean longitude of the Sun in degrees $(L)$ is given as:

$$
L=280.460^{\circ}+0.9856474^{\circ} n
$$


The mean anomaly $(g)$ can be calculated as:

$$
g=357.528^{\circ}+0.9856003^{\circ} n
$$

The ecliptic longitude $(\lambda)$ can be found using $L$ and $g$ :

$$
\lambda=L+1.915^{\circ} \sin (g)+0.020^{\circ} \sin (2 g)
$$

The ecliptic latitude $(\beta)$ is zero:

$$
\beta=0
$$

The obliquity of the ecliptic $(\epsilon)$ is found using $n$ :

$$
\epsilon=23.439^{\circ}-0.0000004^{\circ} n
$$

Given $\epsilon$ and $\lambda$, the direction of the Sun in ECI coordinates is:

$$
\begin{gathered}
X_{E C I}=\cos (\lambda) \\
Y_{E C I}=\cos (\epsilon) \sin (\lambda) \\
Z_{E C I}=\sin (\epsilon) \sin (\lambda) \\
\vec{r}_{\text {sun }}=\left[\begin{array}{l}
X_{E C I} \\
Y_{E C I} \\
Z_{E C I}
\end{array}\right]
\end{gathered}
$$

(Bowen) (The Astronomical Almanac) 


\subsection{Eclipse Conditions}

Modeling the satellite's entrance and exit into the Earth's shadow is important to accurate modeling of the solar radiation pressure torques. Due to the Sun's size and proximity to Earth, it should not be treated as a point source and therefore has a distinct umbra and penumbra parts of its shadow as shown in Figure 4.1.

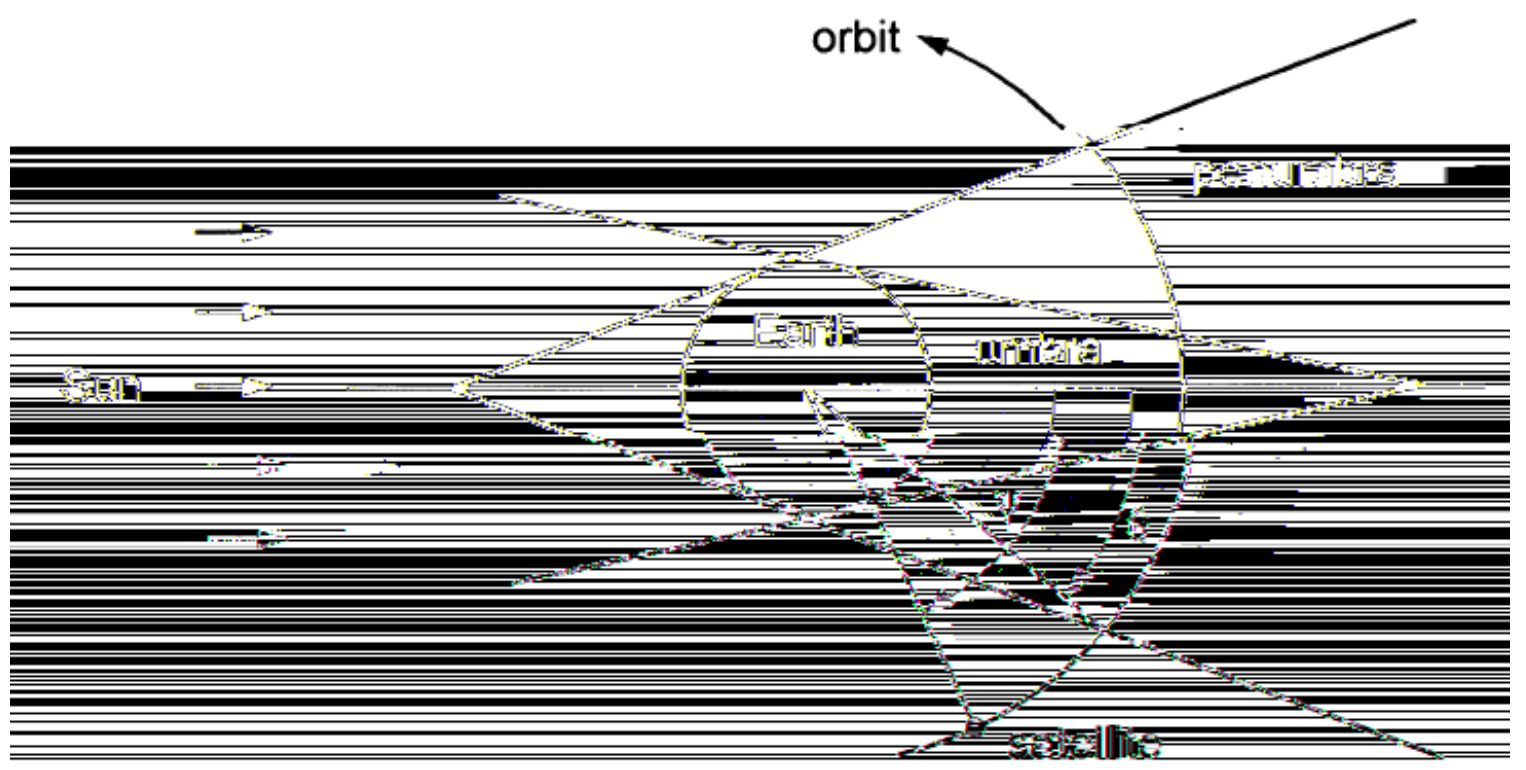

Figure 4.1: Components of Earth's Shadow (Eagle)

To calculate the various parts of Earth's shadow, one must first calculate the half angle the cylindrical projection of the Earth $\left(\theta_{C}\right)$ at the satellite's distance from the Earth in orbit $\vec{r}_{\text {sat }}$.

$$
\theta_{C}=\sin ^{-1}\left(\frac{R_{E}}{\left\|\vec{r}_{\text {sat }}\right\|}\right)
$$


Where $R_{E}$ is the Earth's radius. For more realistic shadow predictions, the radius of the Earth has been increased by $2 \%$ to account for the effect of the Earth's atmosphere on the size of the shadow. The half angle of Earth's umbra $\left(\theta_{U}\right)$ can be calculated as

$$
\theta_{U}=\sin ^{-1}\left(\frac{d_{\text {sun }}-R_{E}}{\left\|\vec{r}_{\text {sun }}\right\|}\right)-\theta_{C}
$$

Where $d_{\text {sun }}$ is the radius of the sun. Similarly, the Earth's penumbra can be calculated as

$$
\theta_{P}=\sin ^{-1}\left(\frac{d_{\text {sun }}+R_{E}}{\left\|\vec{r}_{\text {sun }}\right\|}\right)-\theta_{C}
$$

The location of Earth's shadow can be defined by the negative of the Sun direction vector in ECI, the shadow axis

$$
\hat{r}_{\text {shadow }}=-\frac{\vec{r}_{\text {sun }}}{\left\|\vec{r}_{\text {sun }}\right\|}
$$

The angle of the satellite relative to the shadow axis can be defined as (Eagle)

$$
\phi=\cos ^{-1}\left(\hat{r}_{\text {shadow }} \cdot \hat{r}_{\text {sat }}\right)
$$

\subsection{Atmospheric Model}

An accurate model of atmospheric density is required to model the aerodynamic torques on the spacecraft. The US Naval Research Laboratory's (NRL) Mass Spectrometer and Incoherent Scatter Radar Exosphere (MSISE) model is an empirical, global model of the Earth's atmosphere that spans from sea level to the exosphere. The model calculates composition, temperature, and total mass density. The current model released in 2000, NRLMSISE-00, incorporates the main drivers for the upper atmosphere above $75 \mathrm{~km}$; the 
$10.7 \mathrm{~cm}$ solar radio flux $\left(F_{10.7}\right)$ and the daily geomagnetic index $\left(A_{p}\right)$ (Picone, Drob and Meier). For this simulation, the solar flux and magnetic indices are obtained from ftp.ngdc.noaa.gov using a MATLAB code f107_aph.m created by John A. Smith of CIRES/NOAA that is available online (Smith). Not only does this model serve as a reference for the atmospheric density to model aerodynamic torques on the spacecraft, but will also directly benefit from the data gathered by EXOS. 


\section{Environmental Disturbance Torques}

\subsection{Gravity Gradient Torque}

An asymmetric body subject to a gravitational field will experience a torque tending to align the axis of least inertia with the field direction (Sidi 108).

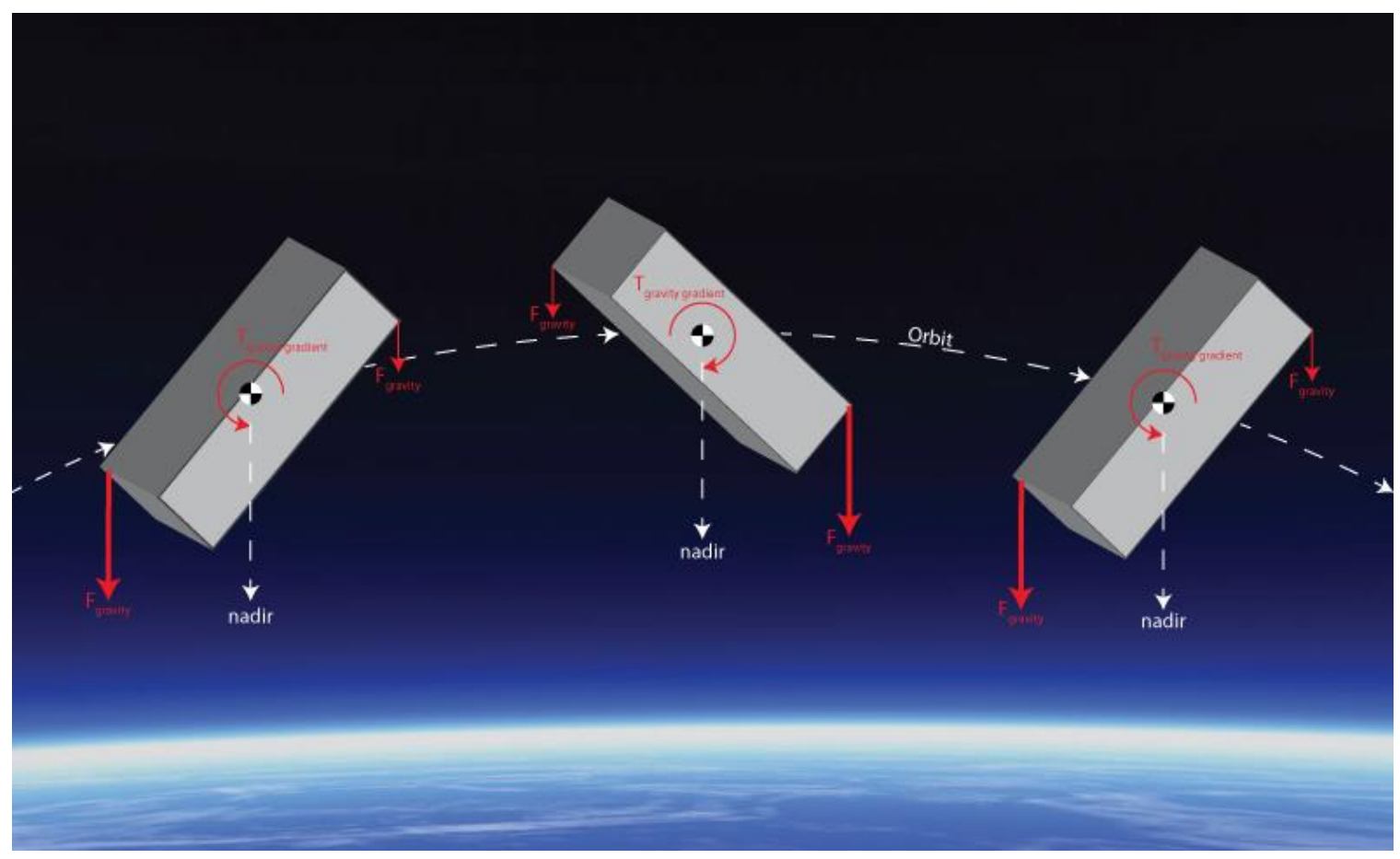

Figure 5.1: Illustration of the gravity gradient effect

According to Newton's Law of Universal Gravitation, in a central gravitational field, portions of a body closer to earth are attracted more strongly than portions further away. Although this force is relatively weak, for an object in LEO, it can be enough to stabilize a satellite to be nadir pointing (Schaub and Junkins). The moon is one example of a naturally gravity gradient stable system. Because the moon has a slight elongation, the same face of the moon points towards the earth as it rotates around it in its orbit (Stern). The gravity gradient torque can be written as (B. Wie 388) 


$$
T_{g g}=3 n^{2} o_{1} \times\left(I_{\text {total }} o_{1}\right)
$$

Where $n$ is the mean motion of the satellite and $o_{1}$ is the basis vector of the orbital frame that is aligned with the orbital position vector and thus the general gravity field defined with respect to the body frame taken from the first column of the direction cosine matrix for the body to orbital transformation $Q_{b o}$.

\subsubsection{Gravity Gradient Stability}

By solving the dynamics equations with the gravity gradient torque and assuming an exponential solution, the characteristic equation leads to a set of 3 inertia parameters known as the Smelt parameters and are bounded between \pm 1 (Hall)

$$
\begin{gathered}
K_{\text {roll }}=\frac{I_{\text {pitch }}-I_{\text {yaw }}}{I_{\text {roll }}} \\
K_{\text {pitch }}=\frac{I_{\text {roll }}-I_{\text {yaw }}}{I_{\text {pitch }}} \\
K_{\text {yaw }}=\frac{I_{\text {pitch }}-I_{\text {roll }}}{I_{\text {yaw }}}
\end{gathered}
$$

The four stability conditions can be expressed as (Hall)

$$
\begin{aligned}
& \text { Condition I: } K_{\text {roll }}>K_{\text {yaw }} \\
& \text { Condition II: } K_{\text {roll }} \cdot K_{\text {yaw }}>0 \\
& \text { Condition III }: 1+3 K_{\text {roll }}+K_{\text {roll }} \cdot K_{\text {yaw }}>0 \\
& \text { Condition IV: }\left(1+3 K_{\text {roll }}+K_{\text {roll }} \cdot K_{\text {yaw }}\right)^{2}-16 K_{\text {roll }} \cdot K_{\text {yaw }}>0
\end{aligned}
$$


Using these conditions, one can construct the stability diagram for a gravity gradient system known as the Smelt Parameter Plane. The conditions bound the stable configurations to exist within the Lagrange and Debra-Delp $\left(\mathrm{D}^{2}\right)$ regions.

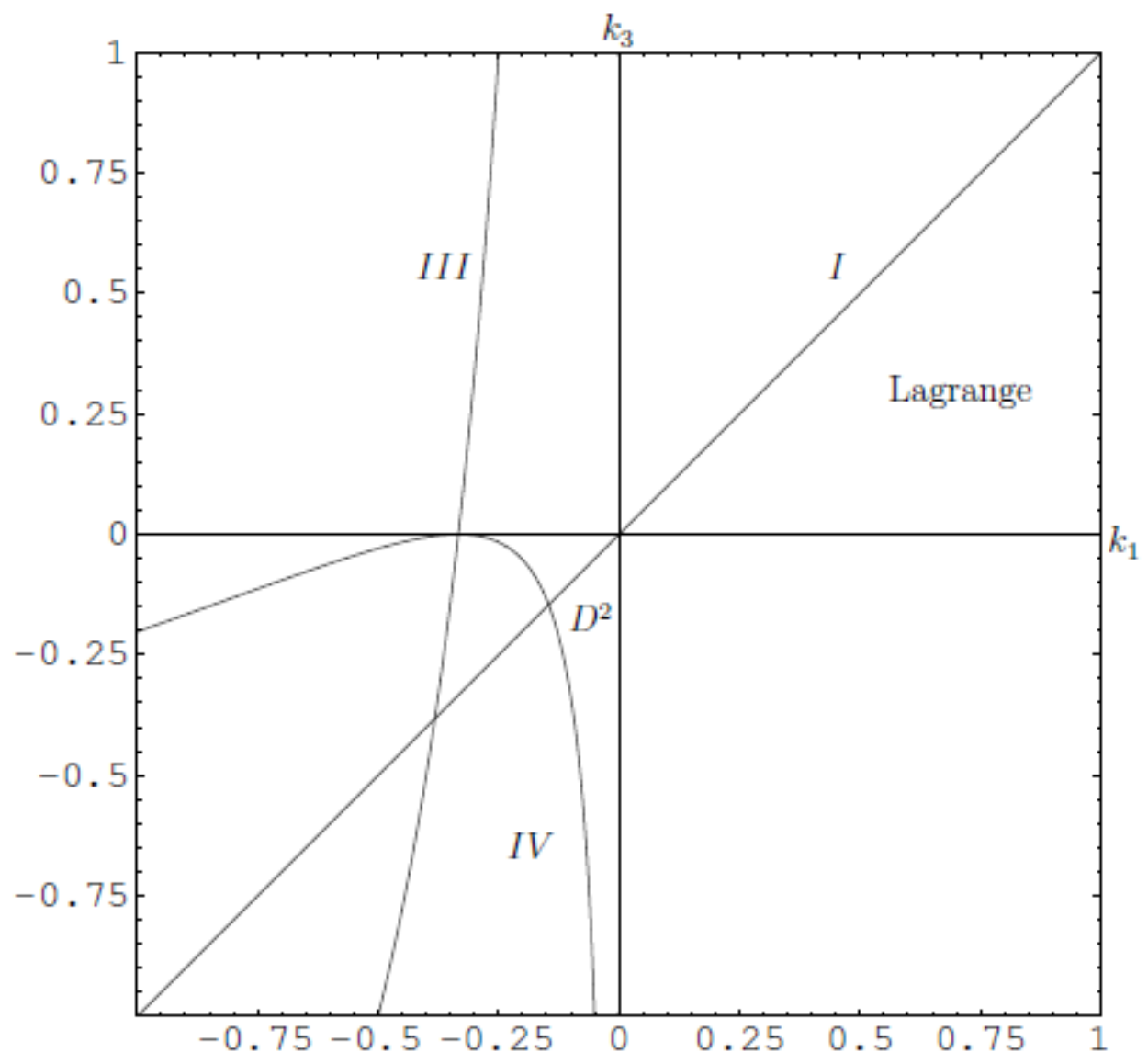

Figure 5.2: The Smelt Parameter Plane (Hall)

\subsection{Magnetic Torque}

The interaction between the magnetic moment $(M)$ produced within a spacecraft and the Earth's magnetic field $(B)$ creates a torque $\left(T_{\text {mag }}\right)$ that can be used for actuation that is the cross product of the two (Sidi 185).

$$
T_{\text {mag }}=M \times B
$$


Because $T_{m a g}$ is the result of a cross product and is therefore always perpendicular to the magnetic field vector $B$, the satellite can only be actuated in the two axes perpendicular to the current magnetic field vector. Although only two axes are controllable at any given time in orbit, the spacecraft experiences two full rotations of the earth's magnetic field vector per orbit making all axes controllable over time. Controllability is also limited since $T_{m a g}$ is proportional to the magnitude of the earth magnetic field vector (Wertz $113)$.

\subsection{Aerodynamic Torque}

Atmospheric density exhibits an overall trend of exponential decay as a function of altitude making torques due to aerodynamic drag a concern mainly for LEO spacecraft. At altitudes that can be qualified as LEO and above, the atmosphere is rarefied and thus the forces created by impacting an object in orbit can be modeled as particles. A simplified model of the torque on a flat panel produced by aerodynamic drag can be written as: (Wertz) (Varma)

$$
T_{\text {aero }}=\frac{1}{2} \rho C_{D} A_{p}\left\|\vec{v}_{\text {rel }}\right\|^{2} \hat{v}_{\text {rel }} \times \vec{r}_{c p}
$$

Where $\rho$ is the atmospheric density in $\mathrm{kg} / \mathrm{m}^{3}, C_{d}$ is the unitless coefficient of drag (typically between 2 and 2.5 for LEO spacecraft), $\hat{n}$ is the outward normal unit vector of the panel, and $r_{c p}$ is the vector from the center of mass of the S/C to the center of

aerodynamic pressure. $\vec{v}_{r e l}$ is the velocity vector of the spacecraft in $\mathrm{km} / \mathrm{s}$. The projected area $\left(A_{p}\right)$ in the equation above can be calculated as 


$$
A_{p}=A \cos (\gamma)
$$

Where $A$ is the surface are of the panel in $\mathrm{m}^{2}$ and $\gamma$ is the angle between the normal vector of the panel $(\hat{n})$ and the relative velocity of the spacecraft $\left(\vec{v}_{r e l}\right)$. The angle $\gamma$ can be calculated as

$$
\gamma=\operatorname{acos}\left(\frac{\hat{n} \cdot \vec{v}_{r e l}}{\|\hat{n}\|\left\|\vec{v}_{r e l}\right\|}\right)
$$

The relative velocity $\left(\vec{v}_{r e l}\right)$ of the spacecraft in the body frame takes into account the rotation of Earth's atmosphere and can be approximated as

$$
\vec{v}_{r e l}=\left[1-\omega_{E} \frac{\|\vec{r}\|}{\|\vec{v}\|} \cos (i)\right] \vec{v}
$$

Where $\vec{r}$ and $\vec{v}$ are the satellite position and velocity in the body frame respectively, $\omega_{E}$ is the angular rate of rotation of the Earth, and $i$ is the orbital inclination (Varma).

\subsection{Solar Radiation Pressure}

The light and radiation emitted by the sun has momentum that exerts pressure on objects exposed to it. The amount of momentum from the solar radiation that is reflected and absorbed is dependent on material properties and can be quantified by the reflectance factor $(q)$, a unitless measure ranging from 0 for perfect absorption to 1 for perfect reflection. A simplified model of the torque on a flat panel produced by solar radiation pressure (SRP) can be written as: (Wertz, Everett and Puschell 571)

$$
T_{S R P}=\frac{\phi}{c} A_{p}(1+q) \hat{n}^{T} \vec{v}_{\text {sun }}\left(\vec{v}_{\text {sun }} \times s_{c p}\right)
$$


Where $\phi$ is the solar output at $1 \mathrm{AU}\left(1366 \mathrm{~W} / \mathrm{m}^{2}\right), c$ is the speed of light in a vacuum $\left(2.99792458 * 10^{8} \mathrm{~m} / \mathrm{s}\right), A$ is the surface area in $\mathrm{m}^{2}, \hat{n}$ is the outward normal unit vector for the panel modeled, $\vec{v}_{\text {sun }}$ is the sun direction in body coordinates, and $s_{c p}$ is the vector from the center of mass of the S/C to the center of solar pressure on the panel (which is assumed to be the same as the center of aerodynamic pressure for these simulations). 


\section{Attitude Determination \& Control Architecture}

The attitude determination and control (ADC) architecture of a CubeSat is limited

primarily by two considerations: budget and power. The budget drives the design because while the NSF space weather grant awarded to ExoCube is one of the larger sources of funding available to university CubeSats, it is still orders of magnitude lower than that of a typical commercial satellite. The power availability drives the design because the mass and volume constraints of the CubeSat standard inherently limit the ability to generate and store power forcing developers to seek out low power active or passive attitude control architectures in order to meet pointing requirements and maximize power available to the payload.

ExoCube needs to maintain pointing in the ram direction within $\pm 10^{\circ}$ with angular rates less than $0.01 \mathrm{deg} / \mathrm{s}$ during science modes. The requirement to point in the ram direction with a relative small angular rate rules out the purely passive options and indicates the need for three-axis pointing. The most common fully active solutions for CubeSats use magnetorquers and reaction wheels for precise three-axis pointing. Magnetorquers create a dipole calculated to exert a torque against the earth magnetic field to actuate the satellite. An active magnetic system requires at least three orthogonal magnetorquers and can achieve accuracies of approximately $\pm 1^{\circ}$ depending on the attitude knowledge (Wertz, Everett and Puschell 574). However, operation of the magnetorquers to continuously maintain pointing would draw excessive power and interfere with the flux of ions into EXOS. Reaction wheels are torque motors with high inertia rotors that can be spun to actuate a satellite (Wertz, Everett and Puschell 579). A zero momentum system 
uses at least 3 orthogonal reaction wheels to actuate a satellite to accuracies of $\pm 1^{\circ}$ and below using alternative actuators like magnetorquers to dump any built up momentum in the wheels (Wertz, Everett and Puschell 574). Although a potentially very accurate agile system, commercial off the shelf (COTS) options for the minimum three reaction wheels required for a zero momentum system are cost prohibitive and draw excessive power. By selecting an over capable attitude control system the developer assumes a lot of unnecessary complexity and cost that ultimately takes away from the primary mission if it doesn't make it entire infeasible. Upon re-examination, ExoCube's pointing requirements are relatively modest in comparison to the capabilities of these fully active three axis control systems and suggest a combination of passive and active elements as the solution.

With power and budget considerations in mind, the design approach for the ADC architecture was to begin with what could be achieved by a purely passive system and then add active elements to tailor a solution to meet the requirements. The passive solutions considered were: permanent magnets, aerodynamically stabilized, and gravity gradient stabilized. A permanent magnet system will align a spacecraft in a polar orbit with the ram direction but was ruled out because the system will flip at the poles and the permanent magnet will interfere with the flux of ionized particles and operation of EXOS. An aerodynamically stabilized system will align with the ram direction providing stability for yaw and pitch motion but roll motion is uncontrolled. A gravity gradient stabilized system will align with the nadir direction providing stability for pitch and roll motion but yaw motion is uncontrolled. Figure 6.1 is a comparison of environmental 
torques by altitude and illustrates that gravity gradient torques dominate aerodynamic torques above an altitude of $400 \mathrm{~km}$. Since the desired orbital regime for ExoCube is between 425 and $650 \mathrm{~km}$ in the thermosphere and lower exosphere, a gravity gradient stabilized system was selected.

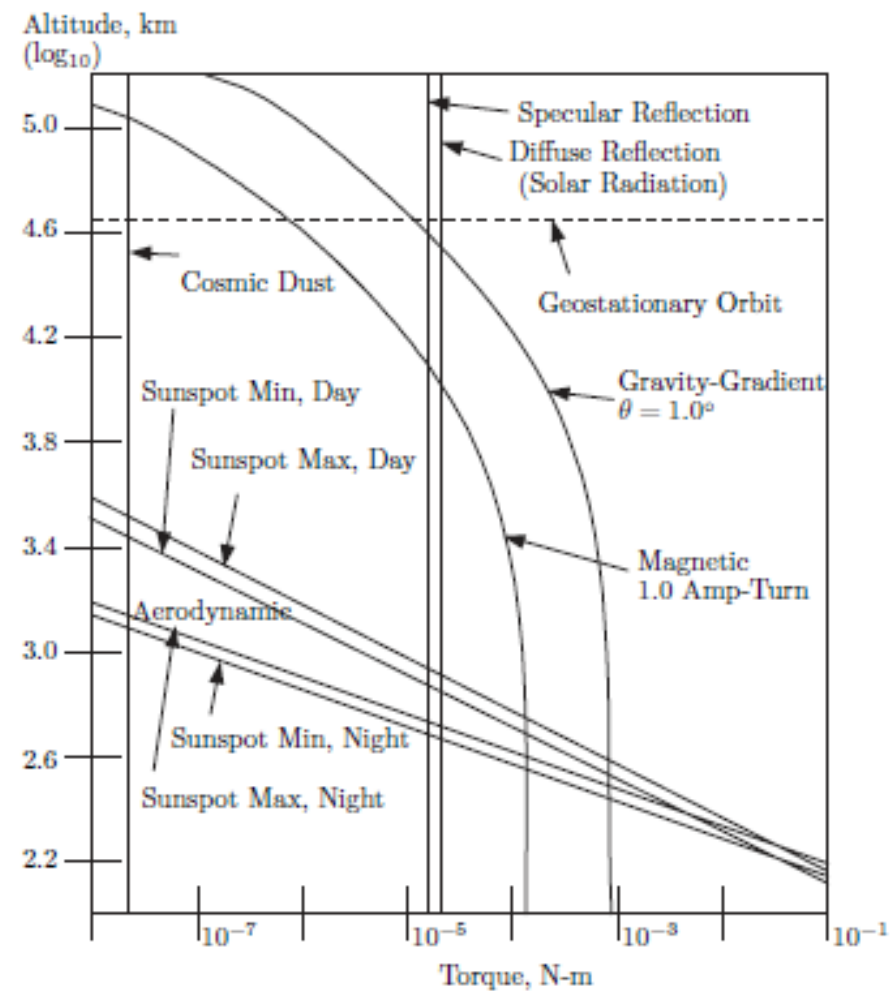

Figure 6.1: Disturbance Torque Regimes (Turner)

Although a $3 \mathrm{U}$ CubeSat with evenly distributed mass is technically gravity gradient stable by the Smelt parameters defined previously, the addition of deployable booms with mass concentrated at the end maximizes the moment of inertia and more importantly makes the center of gravity and alignment of the principal axes and the body axes less sensitive to misalignment errors. Deploying the booms symmetrically from the top and bottom of the $3 \mathrm{U}(+/-\mathrm{z}$ panels) aids in keeping the system aerodynamically balanced by 
keeping the center of pressure close to the center of mass, avoiding the inherent offset in pointing that would be caused by a single boom deployment.

As mentioned previously, a gravity gradient stabilized system has uncontrolled yaw motion. In order to point EXOS in the ram direction, an active control element is required. Since the use of magnetorquers would interfere with EXOS, the gravity gradient system was combined with a momentum bias system.

A momentum bias system can point in the ram direction within $\pm 1^{\circ}$ or better by utilizing a momentum wheel mounted in the pitch axis. The momentum wheel runs at a near constant speed for gyroscopic stability in the roll and yaw disturbances while maintaining pointing in the pitch axis by torqueing the wheel, slightly increasing or decreasing its speed. However, a pure momentum bias system would require periodic momentum dumping, causing frequent interruptions to the operation of EXOS (Wertz, Everett and Puschell 577). By combining a momentum bias with a gravity gradient system, the need for active pitch control and periodic momentum dumping is unnecessary since the gravity gradient system is already stable for pitch motion and the momentum bias will reject disturbances causing yaw drift. The gravity gradient, momentum bias system will still require active magnetic control using magnetorquers for detumbling, initial acquisition of the nominal orientation, wheel spin-up, and re-acquisition of the nominal orientation should ExoCube drift outside its pointing requirements. 


\subsection{Concept of Operations}

The following is a top-level concept of operations for ExoCube as it pertains to the

\section{ADCS.}

- Launch

- During launch ExoCube remains unpowered in the P-POD

- Batteries are fully charged during final integration

- Deploy from P-POD

- Separation switches are closed and ExoCube powers on

- Antenna is deployed via burnwire thirty minutes after deployment

- Satellite Acquisition

- After the antenna deploys, ExoCube will begin transmitting a health beacon at a programmable rate while in a low power state

- System health will be evaluated upon acquisition by the ground station

- Detumble

- Upon confirmation of nominal system health, Exocube will be commanded to detumble

- Attitude Confirmation

- Upon confirmation that ExoCube has detumbled, the Attitude Determination routine will be initiated and an attitude solution downlinked

- After ground analysis of the attitude solution, the nadir pointing camera will be scheduled to take a picture over a landmark to confirm orientation

- Boom Deployment

- After confirming that ExoCube is in an orientation with one of the booms pointing within $\pm 35^{\circ}$ of nadir, the booms will be commanded to deploy via burnwire

- The nadir pointing camera will be commanded to take another picture to confirm deployment and orientation

- Exocube will be given time to settle from any resulting oscillatory motions

- Nominal Orientation Acquisition

- Upon confirmation that ExoCube has successfully deployed its booms and has settled, the Active Magnetic Control routine will be initiated utilizing the Attitude Determination routine to actuate ExoCube into the nominal ram pointing orientation for normal ops

- Wheel Spin-Up

- Upon confirmation that Exocube is in the nominal orientation for normal ops, the pitch momentum wheel will be commanded to slowly spin up while the 
Active Magnetic Control routine maintains position and opposes the torques from the wheel

- ExoCube's orientation is confirmed again by the nadir pointing camera

- Begin Normal Ops

- Upon confirmation of nominal orientation and momentum wheel at speed, ExoCube will begin Normal Ops

- Attitude Maintenance

- Upon analysis of downlinked attitude solutions and determination that ExoCube has drifted outside of its pointing requirements for nominal orientation, the command to suspend Normal Ops will be uplinked.

- The pitch momentum wheel will be commanded to slowly despin while the Active Magnetic Control routine maintains position and opposes the torques from the wheel.

- The Active Magnetic Control routine reacquires the nominal ram pointing orientation.

- The wheel will be commanded to spin up slowly while the Active Magnetic Control routine maintains position and opposes the torques from the wheel.

- ExoCube's orientation is confirmed again by the nadir pointing camera

- Upon confirmation that ExoCube is in the nominal orientation, Normal Ops will be commanded to resume

\subsection{Attitude Determination}

Full three-axis control requires full knowledge of spacecraft orientation and rotational rates. A complete attitude solution requires a suite of sensors that measure rotation rates and use external references to find the spacecraft's orientation. Magnetometers and sun sensors are common solutions used by CubeSat developers and have been selected for ExoCube based largely off of their cost relative to other commercial off the shelf solutions suitable for CubeSats.

Magnetometers measure the magnitude and direction of the Earth's magnetic field relative to the spacecraft and can provide its orientation relative to the inertial frame in two axes when combined with knowledge of the spacecraft's orbital position and the local magnetic field. They are required by the magnetorquers in order to calculate the 
required magnetic dipole to execute commanded torques from the control algorithm. Magnetometers in general have relatively low internal noise but are sensitive to electromagnetic interference from the onboard electronics. As a result, the magnetometers will be placed on the deployable gravity gradient booms to take advantage of the distance from the electromagnetic interference of the main avionics. To simulate the error contribution of magnetometer resolution and noise from the spacecraft electronics, the following equation is used:

$$
b_{\text {meas }}=\operatorname{round}\left(b_{\text {exact }}+\sigma_{b} \operatorname{rand}, \delta b\right)
$$

Where $b_{\text {meas }}$ and $b_{\text {exact }}$ are the measured and actual magnetic field vectors respectively. $\sigma_{b}$ is the standard deviation of the noise used to scale the vector produced by the function randn which returns a random number with a mean of zero and a standard deviation of one. The round function rounds the magnetic field vector with noise to a resolution of $\delta b$ (Guerrant).

Sun sensors measure the direction of the Sun relative to the spacecraft and can provide its orientation relative to the inertial frame in two axes when combined with knowledge of the spacecraft's orbital position and the local sun vector. Provided that the spacecraft is not in eclipse and the local sun vector and Earth magnetic field vector are not coincident, a three-axis solution of its orientation can be found. An attitude determination algorithm is required to combine information from the sensors to produce an attitude solution and propagate the results when the spacecraft is in eclipse or the sun and magnetic field vectors are near coincident. In order to maintain generality for the purpose of simulating 
the performance of the attitude control system, the accumulated errors in resolution and noise in the attitude solution are modeled as follows:

$$
q_{\text {meas }}=\operatorname{round}\left(q_{\text {exact }}+\sigma_{q} \operatorname{randn}, \delta q\right)
$$

Where $q_{\text {meas }}$ and $q_{\text {exact }}$ are the measured and exact body-inertial quaternions respectively. $\sigma_{q}$ is the standard deviation of the noise and $\delta q$ is the resolution to which the quaternion is rounded to (Guerrant).

The angular rate of the satellite can either be obtained directly from gyroscopes or estimated from the change in orientation by the attitude determination algorithm. Knowledge of the angular rate is crucial to propagating the orientation of the satellite when external reference sensors cannot provide a full three-axis solution. Whether the angular rates are provided by gyroscopes or the attitude determination algorithm, the error due to resolution and noise can be modeled as

$$
\omega_{\text {meas }}=\operatorname{round}\left(\omega_{\text {exact }}+\sigma_{\omega} \operatorname{randn}, \delta \omega\right)
$$

Where $\omega_{\text {meas }}$ and $\omega_{\text {exact }}$ are the measured and exact body-inertial angular rates respectively. $\sigma_{\omega}$ is the standard deviation of the noise and $\delta_{\omega}$ is the resolution (Guerrant).

\subsection{Attitude Control Hardware}

\subsubsection{Magnetorquers}

Magnetorquers are relatively simple actuators in that they have no moving parts and consist of coils of wire sometimes wrapped around a ferrous core (Wertz, Everett and Puschell 580). The CubeSats developed by PolySat have all featured air-core 
magnetorquers consisting of concentric traces embedded in a series of layers in the side panels. By embedding them in each of the six side panels of a CubeSat, the magnetorquers provide the capability for redundant actuation in all three axes when in the presence of a magnetic field. The dipole produced by a single magnetorquer can be modeled as

$$
M=i \cdot n \cdot A
$$

Where $M$ is the magnetic dipole in $\mathrm{Am}^{2}, i$ is the current, $n$ is the number of turns in the torque coil, and $A$ is the average area of the enclosed loop. The design for ExoCube builds on the heritage designs of previous successful missions and employs embedded magnetorquers with three layers of twelve turns with an average area of $0.003 \mathrm{~m}^{2}$ and a maximum current of $0.6 \mathrm{~A}$ (Guerrant). The current configuration employs two magnetorquers on each of the sidepanels and one on each of the top and bottom panels resulting in four torquers available to the $Y_{\text {Body }}$ and $Z_{\text {Body }}$ axes and two available to the $X_{\text {Body }}$ axis as shown in Figure 6.2. 


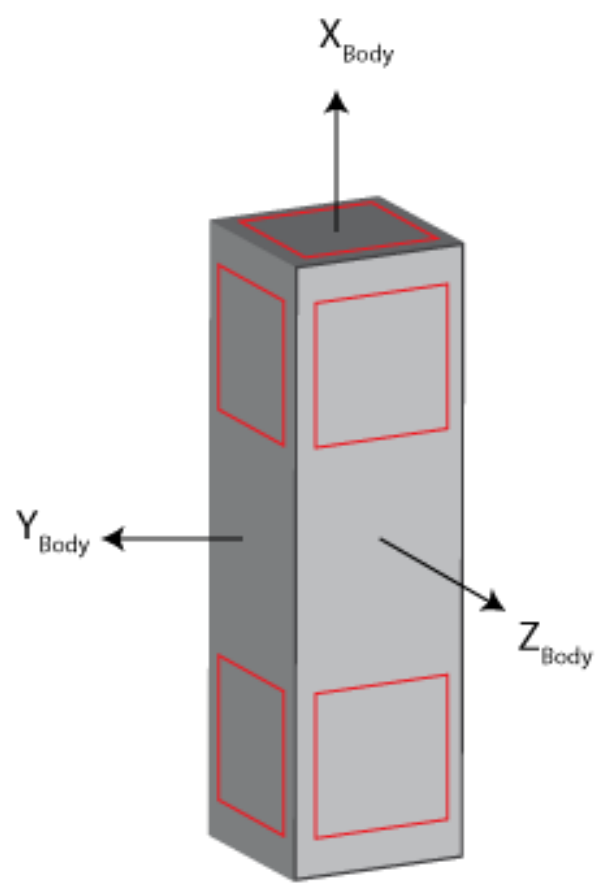

Figure 6.2: Magnetorquer placement on ExoCube (highlighted in red)

As mentioned previously, the maximum torque available is proportional to the Earth's magnetic field vector. The smallest magnetic field intensity $\left(B_{\min }\right)$ available to ExoCube will be $\sim 2.25 \times 10^{-5} T$ at the magnetic equator at an altitude of $650 \mathrm{~km}$, the highest altitude within the range of desired orbits for ExoCube (Wertz, Everett and Puschell 554). In order to guarantee that the magnetorquers have control authority over the most conservative expected disturbance torques, the minimum torque capability is calculated as

$$
\begin{aligned}
T_{\text {mag min }} & =n \cdot i \cdot A \cdot B_{\min } \\
& =(3 \text { layers } \times 12 \text { turns }) \cdot(0.6 \mathrm{~A}) \cdot\left(0.003 \mathrm{~m}^{2}\right) \cdot\left(2.25 \times 10^{-5} \mathrm{~T}\right) \\
& =1.46 \times 10^{-6} \mathrm{~N} \cdot \mathrm{m}
\end{aligned}
$$


This minimum torque capability is compared to the simplified worst case environmental disturbances in Table 6.1

Table 6.1: Summary of worst case environmental torques

\begin{tabular}{|c|c|c|c|}
\hline $\begin{array}{l}\text { Environmental } \\
\text { Torque }\end{array}$ & $\begin{array}{l}\text { Conservative } \\
\text { maximum } \\
{[\mathrm{Nm}]} \\
\end{array}$ & $\begin{array}{l}\text { Simplified Equations } \\
\text { (Wertz) }\end{array}$ & $\begin{array}{l}\text { Conservative } \\
\text { Assumptions }\end{array}$ \\
\hline $\begin{array}{l}\text { Gravity } \\
\text { Gradient }\end{array}$ & $4.24 \times 10^{-7}$ & $T_{g g}=\frac{3}{2} \frac{\mu}{R^{3}}\left|I_{z}-I_{y}\right| \sin (2 \theta)$ & $\begin{array}{l}\text { Lowest acceptable altitude: } \\
R=(425+6378) \mathrm{km} \\
\text { Offset for largest torque: } \\
\theta=45^{\circ}\end{array}$ \\
\hline $\begin{array}{l}\text { Solar } \\
\text { Radiation } \\
\text { Pressure }\end{array}$ & $6.74 \times 10^{-9}$ & $\begin{array}{l}T_{S R P}= \\
\frac{\phi}{c} A(1+q) \cos (i)\left(c_{p}-c_{m}\right)\end{array}$ & $\begin{array}{l}\text { Highest reflectance factor: } \\
q=1 \\
\text { Normal angle of incidence: } \\
i=0^{\circ} \\
\text { Largest allowable offset } \\
\text { (CDS) } \\
\left(c_{p}-c_{m}\right)=0.02 \mathrm{~m}\end{array}$ \\
\hline Aerodynamic & $6.56 \times 10^{-8}$ & $\begin{array}{l}T_{\text {aero }}= \\
\frac{1}{2}\left(\rho \cdot C_{d} \cdot A \cdot\|\vec{v}\|^{2}\right)\left(c_{p}-c_{m}\right)\end{array}$ & $\begin{array}{l}\text { Max atmospheric density: } \\
\rho=2.43 \times 10^{-12} \mathrm{~kg} / \mathrm{m}^{3} \\
\text { Assume max drag } \\
\text { coefficient: } \\
C_{d}=2.4 \quad(2-2.4 \text { typical }) \\
\text { Largest allowable offset } \\
(\mathrm{CDS}): \\
\left(c_{p}-c_{m}\right)=0.02 \mathrm{~m}\end{array}$ \\
\hline Magnetic & $1.46 \times 10^{-6}$ & $T_{\text {mag min }}=n \cdot i \cdot A \cdot B_{\text {min }}$ & $\begin{array}{l}\text { Smallest magnetic field: } \\
B_{\min }=2.25 \times 10^{-5} \mathrm{~T}\end{array}$ \\
\hline
\end{tabular}

As can be seen in Table 6.1, the minimum torque capability is an order of magnitude higher than the gravity gradient torque and several orders of magnitude higher than the worst case aerodynamic and solar radiation pressure torques. This guarantees the magnetorquers can overcome the environmental disturbances in all foreseeable circumstances. 
When a commanded body magnetic dipole is calculated, the components are first divided amongst the corresponding magnetorquers. Next, the requested dipole is converted to a requested current command by the equation for a single magnetorquer defined previously. The requested current is then limited so that no one magnetorquer will exceed the hardware limit of $600 \mathrm{~mA}$. This is accomplished by halving all of the components until all were within the maximum allowed current. This method was chosen because it scales the requested dipole without distorting it and the act of division is a simple bit flip in the software making the current limiter computationally efficient. Finally, a two byte command representing the commanded current is sent to the pulse-width modulator (PWM) that controls the magnetorquer current. A PWM is implemented because a continuously variable current supply for each magnetorquer was not feasible and is the closest feasible approximation. The first byte represents the current direction while the second breaks the full range of 0-600mA into 256 steps (Guerrant). 


\subsubsection{Momentum Wheel}

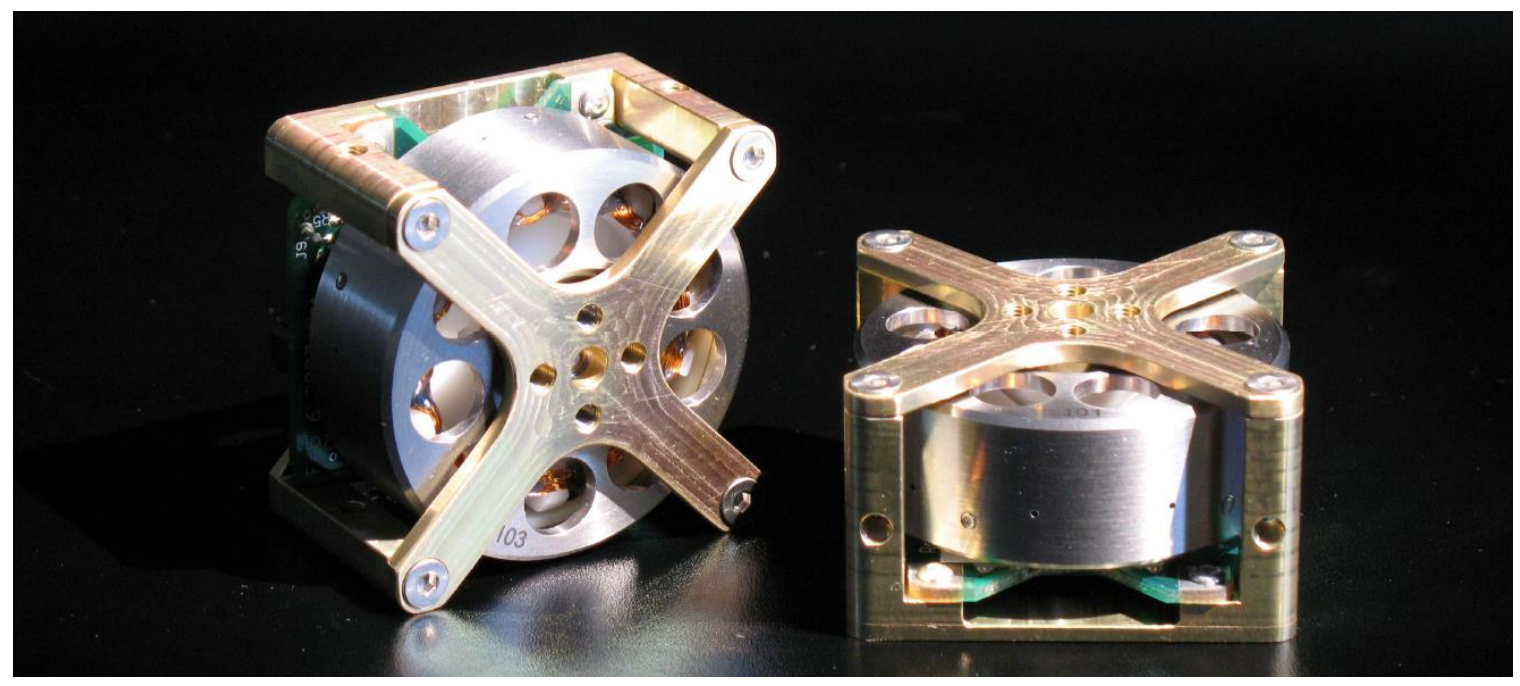

Figure 6.3: Sinclair Interplanetary $10 \mathrm{mNm}$-sec Reaction Wheel

The Sinclair Interplanetary $10 \mathrm{mNm}$-sec reaction wheel has been selected to be used for the momentum bias in ExoCube for its cost, power efficiency, and momentum storage capacity. The RW-0.01-4 has extensive flight heritage and was designed as a joint product between the University of Toronto's Space Flight Laboratory (SFL) and Sinclair Interplanetary. The reaction wheel is unsealed and consists primarily of a stainless steel rotor and a custom-wound motor. The rotor has moment of inertia $\left(I_{\text {wheel }}\right)$ of $2.8004 \times$ $10^{-5} \mathrm{~kg} \cdot \mathrm{m}^{2}$. The SFL has conducted extensive testing to characterize the performance of the reaction wheel and produced the following dynamic equation for the torque produced by the wheel $\left(T_{\text {wheel }}\right)$ : (Philip 38)

$$
T_{\text {wheel }}=I_{\text {wheel }} \cdot \frac{d \omega_{\text {wheel }}}{d t}+c \cdot \omega_{\text {wheel }}
$$


Where $\omega_{\text {wheel }}$ is the angular speed of the wheel and $c$ is the damping coefficient. The sources of friction are the static friction of the rolling ball bearings, the viscous losses of the grease, and loss due to skin friction drag on the rotor. Since the static rolling friction is small relative to the other sources of friction it can be neglected. Viscous friction is proportional to $\omega^{2}$ and is therefore non-linear thus the following equation was developed to model the angular velocity of the reaction wheel (Philip 38).

$$
\omega_{\text {wheel }}(t)=\omega_{0} e^{-\frac{c}{I_{\text {wheel }}} t}
$$

Where $\omega_{0}$ is the initial angular rate of the reaction wheel. When solved for $c$ one can derive an expression for the coefficient of friction for the numerical solver in the simulation based of the ratio of angular rates at times $t_{1}$ and $t_{2}$ of a constant torque curve.

$$
c=\ln \left(\frac{\omega_{2}}{\omega_{1}}\right) \frac{-I_{\text {wheel }}}{t_{2}-t_{1}}
$$

Where $\omega_{1}$ and $\omega_{2}$ are the angular rates of the wheel at times $t_{1}$ and $t_{2}$ respectively.

Although accurate modeling of the viscous friction of the wheel is a valuable feature for a robust simulation, the Sinclair wheel comes with a torque control algorithm that compensates for the torque due to viscous friction to maintain a constant torque while the wheel is spinning up to its desired angular speed. This control mode will be used for the spin up of the momentum wheel and it is therefore more useful to model the noise of the steady state controller as follows.

$$
T_{\text {wheel }}=I_{\text {wheel }} \cdot \frac{d \omega_{\text {wheel }}}{d t}+\sigma_{T} \cdot \operatorname{randn}
$$


Where $\sigma_{T}$ is the standard deviation of the constant torque controller that is reportedly on the order of $10^{-6} N \cdot m$ (Sinclair). 


\section{Attitude Control Algorithms}

To meet the pointing requirements for ExoCube, a control algorithm or set of algorithms are required to actuate the spacecraft into the desired orientation. The concept of operations requires that the system be capable of detumbling, acquiring the desired orientation, spin up the momentum wheel, and maintaining that orientation. The differences in capabilities required at each step suggested a set of specialized algorithms for different phases of the mission rather than a possibly overly capable and complex algorithm for all phases. A B-dot detumbling algorithm and Proportional Derivative (PD) controller were chosen because of their flight heritage in CubeSats and overall simplicity in comparison to other alternatives.

\subsection{B-dot Detumbling Algorithm}

When a CubeSat is deployed from the P-POD, the separation springs and tolerances in the rails can impart a potentially substantial amount of spin. The CubeSat organization estimates that the maximum deployment rotation would be no greater than one revolution per minute. Since magnetometers and magnetorquers are a common and cost effective attitude determination and control solution for CubeSats, a popular method amongst CubeSat developers for detumbling was simply to infer an angular rate from the change in magnetic field direction $(\dot{B})$ registered by magnetometers and attempting to counter that rate through simple proportional feedback control by pulsing the magnetorquers to create an opposing dipole. 


\subsubsection{Control Sequence}

The control sequence can be divided into four distinct steps:

1. Read Attitude Sensors

The magnetic field vector is sampled twice over a short period of time $\delta t$

2. Calculate Attitude and Corrective Pulse

The time derivative of the magnetic field vector is approximated given the two samples through the backwards difference method as follows:

$$
\hat{\dot{B}}=\frac{B^{t}-B^{t-\delta t}}{\delta t}
$$

The required proportional opposing dipole $M_{r e q}$ is calculated from the approximate derivative of the magnetic field vector $\hat{\dot{B}}$ as follows:

$$
M_{\text {req }}=-K \hat{\dot{B}}
$$

Where $K$ is the proportional gain found through trial and error. The calculation itself takes time for the processor to handle the information and is accounted for in the simulations as a delay of 0.1 seconds as a conservative estimate.

\section{Pulse Magnetorquers}

The required dipole in body coordinates is then divided amongst the magnetorquers as a calculated current achieved through pulse width modulation over the same length of time as the measurement phase $\delta t$.

\section{Wait for Hysteresis to Dissipate}

The magnetorquer pulse creates a residual magnetic field that can significantly impact magnetometer readings. A small but conservative delay of 0.1 seconds before reading the magnetometers again is added to allow for the induced field to dissipate.

This sequence continues so long as B-dot is active until it is overridden or the convergence criteria are met. The condition for convergence for the B-dot controller is defined as when the mean of the last five normalized $\dot{B}$ measurements is below a predefined tolerance.

$$
\dot{\bar{B}}=\frac{1}{5}\left(\dot{B}_{t-4}+\dot{B}_{t-3}+\dot{B}_{t-2}+\dot{B}_{t-1}+\dot{B}_{t}\right)
$$


Where $t$ is the most recent calculation of $\dot{B}$. This averaging helps smooth out noise from the magnetometers and ensures that the satellite has in fact detumbled.

\subsection{Three-Axis Control Algorithm}

Once ExoCube has successfully detumbled and deployed its booms, the system needs a more complicated control algorithm to acquire and maintain the nominal ram pointing orientation. The need for a capable but simple three axis controller was the subject of Dan Guerrant's thesis in which a Proportional Derivative (PD) controller was selected over alternatives like a Linear Quadratic Regulator (LQR) for several reasons. First, a PD controller can be designed using classical control theory and therefore gain selection is relatively intuitive. Second, the PD controller does not depend on linearized equations of motion and therefore is capable of large angle maneuvers by incorporating the nonlinear dynamics.

In the design of any controller, a careful definition of "optimal" must be agreed upon in order to proceed. In the case of re-orienting a satellite, one approach is to define the “optimal” maneuver as Euler's eigenaxis rotation which provides the shortest angular path between two orientations. The eigenaxis rotation that provides this optimal path is defined by the error quaternion.

The quaternion error $\left(q_{e}\right)$ is the quaternion describing the rotation required to actuate from the actual orientation of the satellite $\left(q_{a}\right)$ to the desired orientation $\left(q_{d}\right)$ (B. Wie 320) 


$$
q_{e}=\left[\begin{array}{cccc}
q_{d 4} & q_{d 3} & -q_{d 2} & -q_{d 1} \\
-q_{d 3} & q_{d 4} & q_{d 1} & -q_{d 2} \\
q_{d 2} & -q_{d 1} & q_{d 4} & -q_{d 3} \\
q_{d 1} & q_{d 2} & q_{d 3} & q_{d 4}
\end{array}\right]\left[\begin{array}{l}
q_{a 1} \\
q_{a 2} \\
q_{a 3} \\
q_{a 4}
\end{array}\right]
$$

This equation is the result of successive quaternion rotations using quaternion multiplication and inversion rules. If the actual and desired orientations are aligned, the error quaternion will be $q_{e}=[0,0,0,1]^{T}$.

Wie, Weiss, and Arapostathis proposed a quaternion feedback regulator for spacecraft eigenaxis rotational maneuvers (Wie, Weiss and Arapostathis). The controller consists of linear quaternion error feedback, linear body rate feedback and a non-linear body rate feedback term that counteracts the gyroscopic coupling torque and is defined as follows:

$$
T_{\text {req }}=-\omega^{\times} I \omega-D \omega-K q_{e}
$$

Where $\omega^{\times} I \omega$ is the non-linear body rate feedback term and $D$ and $K$ are the rate (derivative) and orientation (proportional) gains respectively (Wie, Weiss and Arapostathis). The gyroscopic decoupling term is not necessary for slow rotational maneuvers but may be desirable to counter the natural gyroscopic coupling by the control torque.

\subsubsection{Global Stability}

Wie et al. prove global stability given the generic case of the closed loop system for the control law with general $D$ and $K$ matrices,

$$
I \dot{\omega}=\omega^{\times} I \omega-\mu\left(\omega^{\times} I \omega\right)-D \omega-K q
$$




$$
\begin{gathered}
\dot{\vec{q}}=\frac{1}{2}\left[q_{4} \omega_{b i}-\omega_{b i}^{\times} \vec{q}\right] \\
\dot{q}_{4}=-\frac{1}{2} \omega_{b i}^{T} \vec{q}
\end{gathered}
$$

Where $\mu=1$ means that the control torque exactly counteracts the gyroscopic coupling torque and $\mu=0$ means that only the linear portions of the control law are used. For simplicity the desired quaternion is set as $q_{d}=\left[\begin{array}{llll}0 & 0 & 0 & 1\end{array}\right]^{T}$ so that the error quaternion can be replaced by the current attitude quaternion.

Assuming $K^{-1}$ exists and $K^{-1} I$ is positive definite, the following Lyapunov function can be defined:

$$
\begin{aligned}
& V=\frac{1}{2} \omega^{T} K^{-1} I \omega+q_{1}^{2}+q_{2}^{2}+q_{3}^{2}+\left(q_{4}-1\right)^{2} \\
& =\frac{1}{2} \omega^{T} K^{-1} I \omega+2\left(1-q_{4}\right)
\end{aligned}
$$

Where $V$ is positive definite and asymptotically unbounded by $\omega$.

The time derivative of the Lyapunov function is

$$
\dot{V}=\omega^{T} K^{-1} I \omega+\frac{1}{2} \omega^{T} K^{-1} I \dot{\omega}-2 \dot{q}_{4}
$$

Assuming $K^{-1} I=\left(K^{-1} I\right)^{T}$, we can calculate $\dot{V}$ along the system trajectories as

$$
\begin{aligned}
\dot{V} & =\omega^{T} K^{-1} I \dot{\omega}-2 \dot{q}_{4} \\
& =-\omega^{T} K^{-1} D \omega+(1-\mu) \omega^{T} K^{-1} \omega^{\times} I \omega
\end{aligned}
$$


The second term of the equation above is identically zero when:

1. There is precise cancellation of the gyroscopic coupling torque $(\mu=1)$ or,

2. There is no cancellation of the gyroscopic coupling torque and selection of the orientation gain $K$ is such that

$$
K^{-1}=\alpha I+\beta \text { identity }
$$

Where $\alpha$ and $\beta$ are nonnegative scalars and $I$ is a $3 \times 3$ identity matrix

Using the second condition and plugging it back into the time derivative of the Lyapunov function we get

$$
\begin{aligned}
\omega^{T} K^{-1} \omega^{\times} I \omega & =\omega^{T}(\alpha I+\beta \text { identity }) \omega^{\times} I \omega \\
& =\alpha(I \omega)^{T} \omega^{\times}(I \omega)+\beta \omega^{T} \omega^{\times} I \omega
\end{aligned}
$$

Since $\omega^{\times}=\left(\omega^{\times}\right)^{T}$, the first term in the equation above is identically zero. Given the definition of $\omega^{\times}, \omega^{\times} \omega=\omega^{T} \omega^{\times} \equiv 0$, and the second term in the equation above is identically zero. The second condition guarantees that $K^{-1}$ exists and $K^{-1} I$ is symmetric and positive definite. Therefore it can be shown that under condition 1 or 2 that

$$
\dot{V}=-\omega^{T} K^{-1} D \omega
$$

Global stability is guaranteed if $K^{-1} D>0$. Global stability is therefore guaranteed by selecting $D$ to be defined as

$$
D=d I
$$

Where $d$ is a positive scalar (Wie, Weiss and Arapostathis). 


\subsubsection{Gain Selection}

In order to meet the conditions for global stability for an eigenaxis rotation, the gains selected should satisfy

$$
\begin{aligned}
& K=k I \\
& D=d I
\end{aligned}
$$

Where $k$ and $d$ are both positive scalars. If $\lambda$ is a unit vector along the eigenaxis, the vector portion of the quaternion rotation through the principal angle $\phi$ is

$$
\vec{q}=\lambda \sin \left(\frac{\phi}{2}\right)
$$

If the angular rate $\omega$ is assumed to be small enough to neglect the gyroscopic term, Euler's equation for rotational motion with the controller can be approximated by

$$
\left(\ddot{\phi}+d \dot{\phi}+k \sin \left(\frac{\phi}{2}\right)\right) I \lambda=0
$$

Because the approximation is for an eigenaxis rotation, the angular rate can be written as $\omega=\dot{\phi} \lambda$. Since $I \lambda \neq 0$, the equation above can be reduced to

$$
\ddot{\phi}+d \dot{\phi}+k \sin \left(\frac{\phi}{2}\right)=0
$$

For $\phi \leq 90^{\circ}$, we can approximate $\sin (\phi / 2)$ as $\phi / 2$ giving the linear second order equation

$$
\ddot{\phi}+d \dot{\phi}+k \frac{\phi}{2}=0
$$


Where the damping ratio $(\zeta)$ and the natural frequency $\left(\omega_{n}\right)$ satisfy

$$
\begin{gathered}
d=2 \zeta \omega_{n} \\
\frac{k}{2}=\omega_{n}^{2}
\end{gathered}
$$

It should be noted that due to the non-linear effects of $\sin (\phi / 2)$, the normal equation for settling time $\left(t_{s}=4 / \zeta \omega_{n}\right)$ should be modified to $t_{s}=8 / \zeta \omega_{n}$ for $\phi \cong 180^{\circ}$ (Wie, Weiss and Arapostathis).

\subsubsection{Moment of Inertia Uncertainty}

CubeSats inherently have relatively large uncertainties in the knowledge of the actual moments of inertia (MOI) of the flight unit due largely to the low cost manufacturing methods and lack of access to the proper measuring equipment. Accurate CAD modelling offers the best insight into what the final MOI will be. To be thoroughly robust, the three axis controller needs to be globally stable given uncertainty in the MOI and the gains selected should be independent of these uncertainties. Wie et al. proves global stability of the controller given MOI uncertainty through the following process (Wie, Weiss and Arapostathis).

If $I_{n}$ is the nominal value of the inertia matrix and $\Delta I$ is the uncertainty, then Euler's equation of rotational motion with the control law incorporated can be written as

$$
\left(I_{n}+\Delta I\right) \dot{\omega}=\omega^{\times}\left(I_{n}+\Delta I\right) \omega-\omega^{\times} I_{n} \omega-D \omega-K q
$$


Where the gyroscopic torque is not completely cancelled as a result of the uncertainty. The above equation can be rewritten as

$$
I \dot{\omega}=\omega^{\times} \Delta I \omega-D \omega-K q
$$

Where $I=\left(I_{n}+\Delta I\right)$. Given the conditions for global stability defined previously, global stability can be guaranteed provided $K^{-1}=\beta$ identity or equivalently $K=k$ identity regardless of the knowledge of $\Delta I$. The cost of selecting $K=k$ identity instead of $K=$ $k I$ is that the rotation will be sub-optimal as it will not be performed around the eigenaxis (Wie, Weiss and Arapostathis).

\subsubsection{Pseudo-Reverse Cross Product}

As mentioned previously, not all axes are controllable at any given time when using active magnetics. In order to conserve energy, the best-fit approximation of the reverse of the cross product defining the magnetic torque is used to produce the commanded magnetic dipole $\left(M_{\text {command }}\right)$ for only the portion of the requested torque vector $\left(T_{\text {req }}\right)$

that is perpendicular to the magnetic field vector in body coordinates $\left(B_{\text {body }}\right)$ (Guerrant 24) (Guelman, Waller and Shiryaev).

$$
M_{\text {command }}=\frac{B_{\text {body }} \times T_{r e q}}{\|B\|^{2}}
$$

\subsubsection{Control Sequence}

The control sequence is divided into four distinct steps:

1. Read Attitude Sensors 
The magnetometers and sun sensors are read. The act of polling all the sensors will take time and is accounted for in the simulations as a delay of 0.1 seconds as a conservative estimate.

2. Calculate Attitude and Corrective Pulse

The calculation itself takes time for the processor to handle the information and is accounted for in the simulations as a delay of 0.1 seconds as a conservative estimate.

3. Pulse Magnetorquers

The required dipole in body coordinates is then divided amongst the magnetorquers as a calculated current achieved through pulse width modulation over the same length of time as the measurement phase $\delta t$.

4. Wait for Hysteresis to Dissipate

The magnetorquer pulse creates a residual magnetic field that can significantly impact magnetometer readings. A small but conservative delay of 0.1 seconds before reading the magnetometers again is added to allow for the induced field to dissipate.

This sequence continues so long as the PD controller is active until it is overridden or the convergence criteria are met. The condition for convergence for the PD controller is defined as when the norm of the angular rate error $\left(\omega_{\text {err }}\right)$ and the orientation error are within the predefined bounds.

$$
\begin{aligned}
& \left\|\omega_{\text {err }}\right\|<\omega_{\text {tol }} \\
& 2 \cos ^{-1}\left(q_{4 \text { err }}\right)<<_{\text {tol }}
\end{aligned}
$$

Where $\omega_{t o l}$ and $\angle_{t o l}$ are the acceptable tolerances for the desired angular rate and orientation respectively (Guerrant). 


\section{Simulation and Results}

The attitude control simulation is based on the previous code and work of several students in the lab (Guerrant) (Sturm II) (Bender). This simulation is the culmination of their combined efforts with modifications to produce a high fidelity tool for evaluating the attitude control design and performance of ExoCube and for many CubeSats to come.

\subsection{Assumptions}

Although every effort has been made to make the simulation as accurate as possible, several key assumptions have been made. The following assumptions were deemed sufficient for the level of accuracy required or necessary to computational efficiency.

- The spacecraft is a rigid body.

- The principal inertia axes are aligned with the body axes.

- $\quad$ J2 spherical harmonics perturbations are included but third body perturbations are neglected.

- The error from onboard orbital position knowledge is negligible

- The error from misalignment of sensor mounting is negligible

- The momentum wheel is mounted with its spin axis aligned with the body pitch axis with negligible misalignment.

- The avionics is capable of performing attitude determination and control calculations in 0.1 seconds

- The electronic power subsystem (EPS) is capable of providing $0.6 \mathrm{~A}$ per magnetorquer at all times.

\subsection{Satellite Specifications}

The satellite simulated in the following results represents the most current design of ExoCube. The stowed and deployed moments of inertia listed in the following sections are drawn from CAD models. To model the aerodynamic and solar radiation pressure torques, the spacecraft was assumed to be comprised of flat panels with centers of 
pressure in the geometric center of each. Per the CDS, the center of gravity is constrained to be within a $2 \mathrm{~cm}$ radius sphere at the center of geometry and is adjustable within the code (Munakata). For the stowed configuration, six panels representative of the area for each of the six sides of the $3 \mathrm{U}$ were used. For the deployed configuration, a conservative estimate of the deployable booms was added to the model deployed in their intended configuration through and angle of 194 degrees as pictured in Figure 8.1.

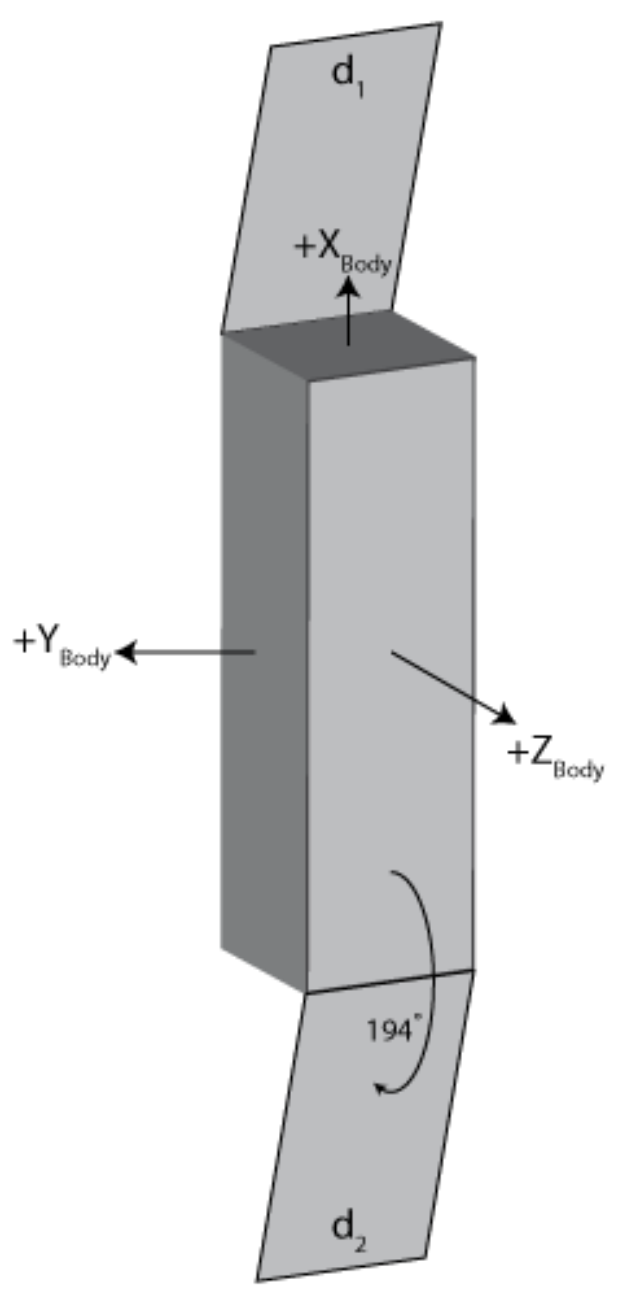

Figure 8.1: Panel representation of spacecraft in deployed configuration 
The following table summarizes the areas $(A)$ of each of the panels shown in Figure 8.1 as well as the vectors $\left(\vec{r}_{c p}\right)$ defining the center of pressure of each panel in relation to the overall center of geometry.

Table 8.1: Summary of Satellite Configurations for Simulation

\begin{tabular}{|c|c|c|c|c|c|c|c|c|}
\hline & \multicolumn{7}{|c|}{ Deployed Configuration } \\
\cline { 2 - 9 } & $+X_{\text {Body }}$ & $+Y_{\text {Body }}$ & $+Z_{\text {Body }}$ & $-X_{\text {Body }}$ & $-Y_{\text {Body }}$ & $-Z_{\text {Body }}$ & $d_{1}$ & $d_{2}$ \\
\hline$A\left[m^{2}\right]$ & 0.01000 & 0.03405 & 0.03405 & 0.01000 & 0.03405 & 0.03405 & 0.006053 & 0.006053 \\
\hline$\vec{r}_{c p}[m]$ & {$\left[\begin{array}{c}0.15 \\
0 \\
0\end{array}\right]$} & {$\left[\begin{array}{c}0 \\
0.05 \\
0\end{array}\right]$} & {$\left[\begin{array}{c}0 \\
0 \\
0.05\end{array}\right]$} & {$\left[\begin{array}{c}-0.15 \\
0 \\
0\end{array}\right]$} & {$\left[\begin{array}{c}0 \\
0.05 \\
0\end{array}\right]$} & {$\left[\begin{array}{c}0 \\
0 \\
-0.05\end{array}\right]$} & {$\left[\begin{array}{c}0.2953 \\
0 \\
0.0138\end{array}\right]$} & {$\left[\begin{array}{c}-0.2953 \\
0 \\
-0.0138\end{array}\right]$} \\
\hline
\end{tabular}

The coefficient of drag $\left(C_{D}\right)$ for LEO spacecraft typically varies between 2-2.5 and thus a conservative estimate of 2.5 was applied. Similarly, a conservative estimate of the solar reflectance factor of 0.8 was applied to the calculation of the solar radiation pressure torques (Wertz, Everett and Puschell). 


\subsection{Guide to B-dot Controller Results Plots}

In the following sections, several key simulations have been selected from the many run to demonstrate various aspects of the performance of the B-dot controller. The results of each simulation are summarized in a single figure containing six subplots a-f which are detailed in Table 8.2. The x-axis of all subplots are given in minutes. The line of each subplot except for the quaternions are colored accordingly as

$$
X_{\text {Body }} \text { :Blue } \quad Y_{\text {Body }}: \text { Green } \quad Z_{\text {Body }}: \text { Red }
$$

For the quaternion plots the vector components are represented by the above colors and approximate the body orientation when close to alignment with the reference frame. The scalar component of the quaternion is represented by the cyan colored line. 
Table 8.2: Summary of B-dot controller results plots

\begin{tabular}{|c|c|c|c|c|c|}
\hline & $\begin{array}{l}\text { Magnetic Field in the Orbital Frame } \\
\text { The magnetic field components in the } \\
\text { orbital frame from the IGRF model } \\
\text { Represents the information available to } \\
\text { the satellite when a magnetic lookup } \\
\text { table and orbital position are combined } \\
\text { Peaks indicate poles and are often } \\
\text { associated with localized instabilities. }\end{array}$ & $\begin{array}{l}\text { b) } \\
- \\
-\end{array}$ & $\begin{array}{l}\text { Magnetic Field in the Body Frame } \\
\text { The magnetic field components as seen } \\
\text { by the magnetometers in the body frame } \\
\text { Gray lines represent the measured values } \\
\text { and include any resolution or noise } \\
\text { errors. }\end{array}$ & & $\begin{array}{l}\text { B-dot } \\
\text { The components of the change in the } \\
\text { magnetic field as calculated by the B-dot } \\
\text { algorithm from the measured magnetic } \\
\text { field. }\end{array}$ \\
\hline d) & $\begin{array}{l}\text { Torquer Current Provided } \\
\text { Commanded torquer currents bounded } \\
\text { by the current limiter proportional to the } \\
\text { calculated B-dot value. }\end{array}$ & - & $\begin{array}{l}\text { Inertial Angular Rates } \\
\text { The components of the actual simulated } \\
\text { values for the body-inertial angular rates } \\
\text { in degrees per second. } \\
\text { Note that the B-dot controller indirectly } \\
\text { controls the body-inertial rates by } \\
\text { approximating them through the change } \\
\text { in magnetic field over short periods of } \\
\text { time. }\end{array}$ & f) & $\begin{array}{l}\text { Inertial Quaternions } \\
\text { The components of the actual simulated } \\
\text { values of the body-inertial quaternion. } \\
\text { Note that the B-dot controller does not } \\
\text { control orientation and this plot is shown } \\
\text { purely for reference. }\end{array}$ \\
\hline
\end{tabular}




\subsection{Detumbling}

Many simulations were run in order to optimize the three main control parameters: gain, pulse length, and convergence criteria. Upon finding the optimum of each control parameter, error in the magnetometer readings was included to evaluate the robustness of the B-dot controller and its performance. For each of the detumbling simulations, a polar orbit (inclination of $95^{\circ}$ ) in the middle of ExoCube's desired altitude range (475 $\mathrm{km}$ ) was selected as a representative test case with large magnetic field variation. The conservative estimate of the 3U's stowed moment of inertia that was used in the simulations is

$$
I_{\text {total }}=\left[\begin{array}{ccc}
0.0053 & 0 & 0 \\
0 & 0.0336 & 0 \\
0 & 0 & 0.0336
\end{array}\right]
$$

As mentioned previously, the maximum expected rotation rate imparted by deployment from the P-POD would be one revolution per minute (Guerrant). Thus the initial rate for the simulation was set as

$$
\omega_{0}=\left[\begin{array}{lll}
\pi / 30 & 0.05 & -0.05
\end{array}\right](\mathrm{rad} / \mathrm{s})
$$

Where the maximum rotation is applied about the $b_{1}$ axis of the satellite which has the least magnetorquers and the other terms are perturbation velocities. For simplicity, the initial orientation was set so that the body frame was aligned with the inertial frame

$$
q_{0}=\left[\begin{array}{llll}
0 & 0 & 0 & 1
\end{array}\right]
$$




\subsubsection{Gain Selection}

The range of acceptable gains was found by running the simulation with a wide range of values for $K_{b d o t}$. The simulations were run for one and a half orbits which was deemed a reasonable period for detumbling based off of previous missions. No error was introduced into the magnetometer readings and the pulse lengths were set to 9.9 seconds based on previous designs. The angular rate and total current draw for each gain tested was recorded and shown in Figure 8.2.

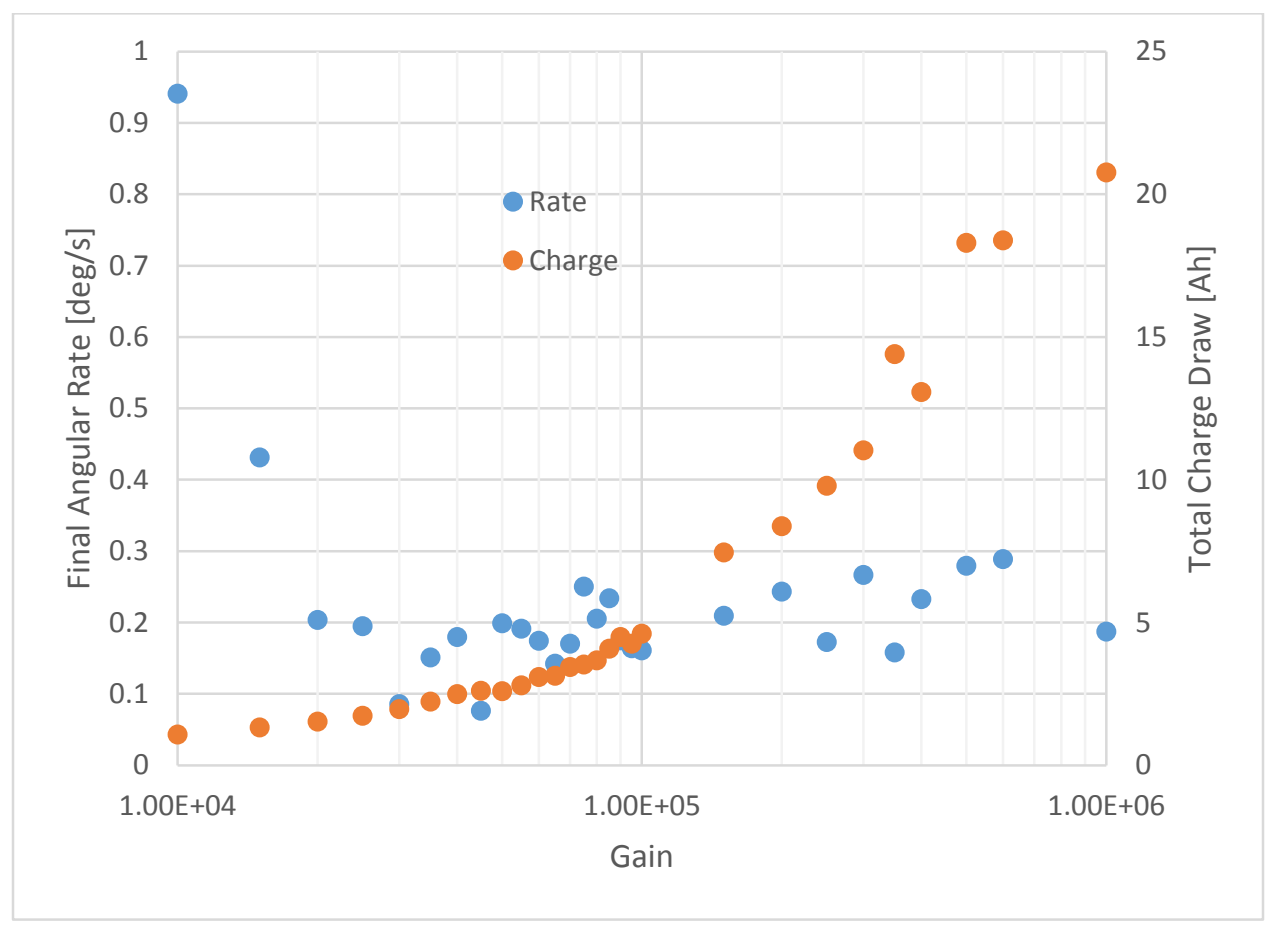

Figure 8.2: B-dot performance for selected range of gains

As can be seen in Figure 8.2, the angular rate after 1.5 orbits remains fairly consistent at an average of $0.1823 \mathrm{deg} / \mathrm{s}$ save for the gains lower than $2 \times 10^{4}$ which appear to be underpowered and didn't converge within 1.5 orbits. Higher gains show faster initial settling times at the cost of power as evident by the total current draw shown in the 
figure. However, for gains above $3 \times 10^{5}$, the simulations show evidence of local instabilities that occur when the satellite passes over the poles and although the controller recovers, this behavior draws unnecessary amounts of current and is thus undesirable. The acceptable range of gains given the conditions of the simulation is therefore between $2 \times 10^{4}$ and $3 \times 10^{5}$. Simulations exemplifying an optimal case, a sub-optimal case, and local instabilities are shown in detail in the following sections. The sub-optimal case illustrates the realistic performance given the uncertainties in the actual satellite. 


\subsubsection{Optimal Case}
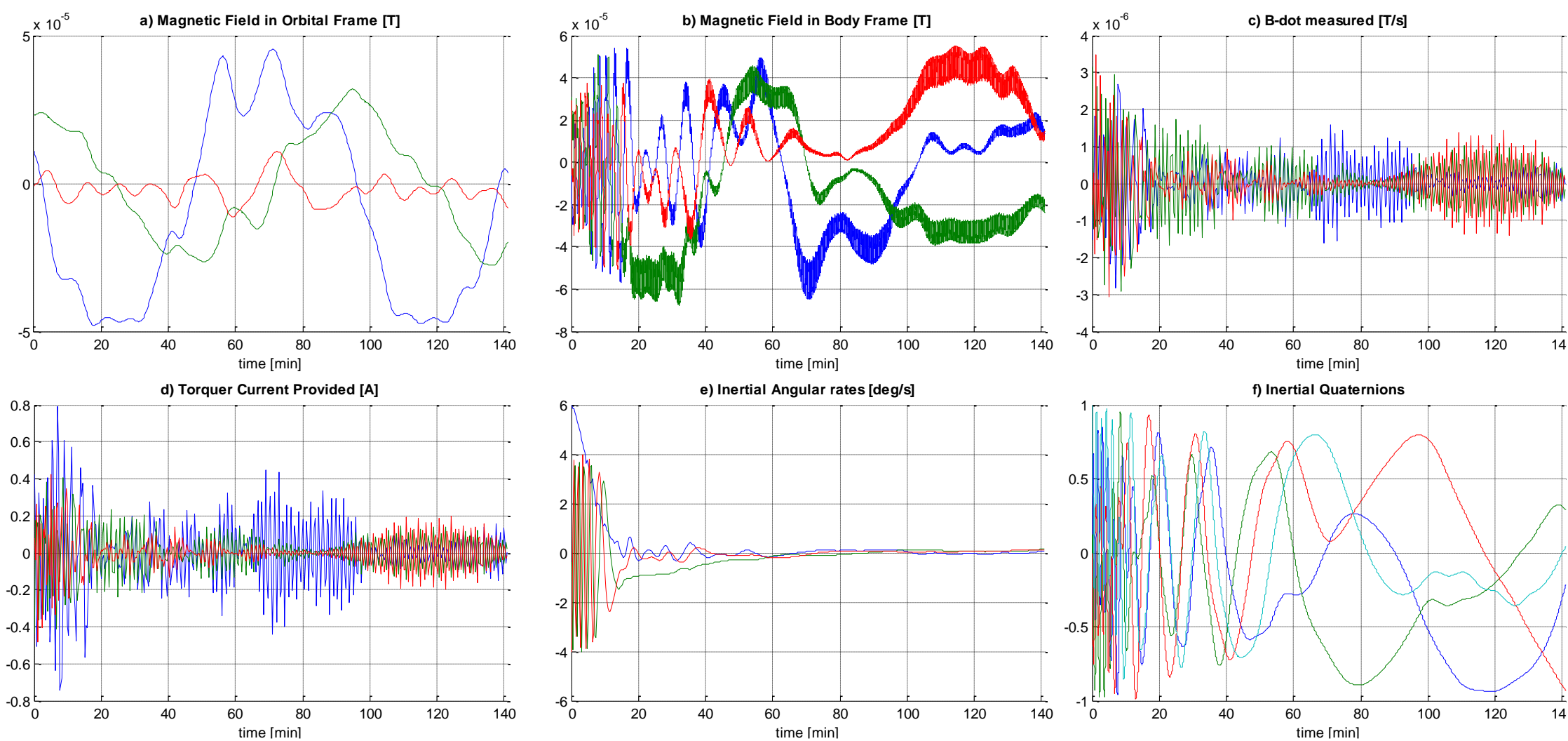

Figure 8.3: Optimal gain performance of the B-dot controller

\begin{tabular}{|c|c|c|}
\hline$K_{b d o t}$ & Pulse Length: $d t[\mathrm{~s}]$ & Final Rate: $\|\omega\|[\mathrm{deg} / \mathrm{s}]$ \\
\hline $\mathbf{6} \times \mathbf{1 0}^{\mathbf{4}}$ & 9.9 & 0.1742 \\
\hline
\end{tabular}




\subsubsection{Sub-Optimal Case}
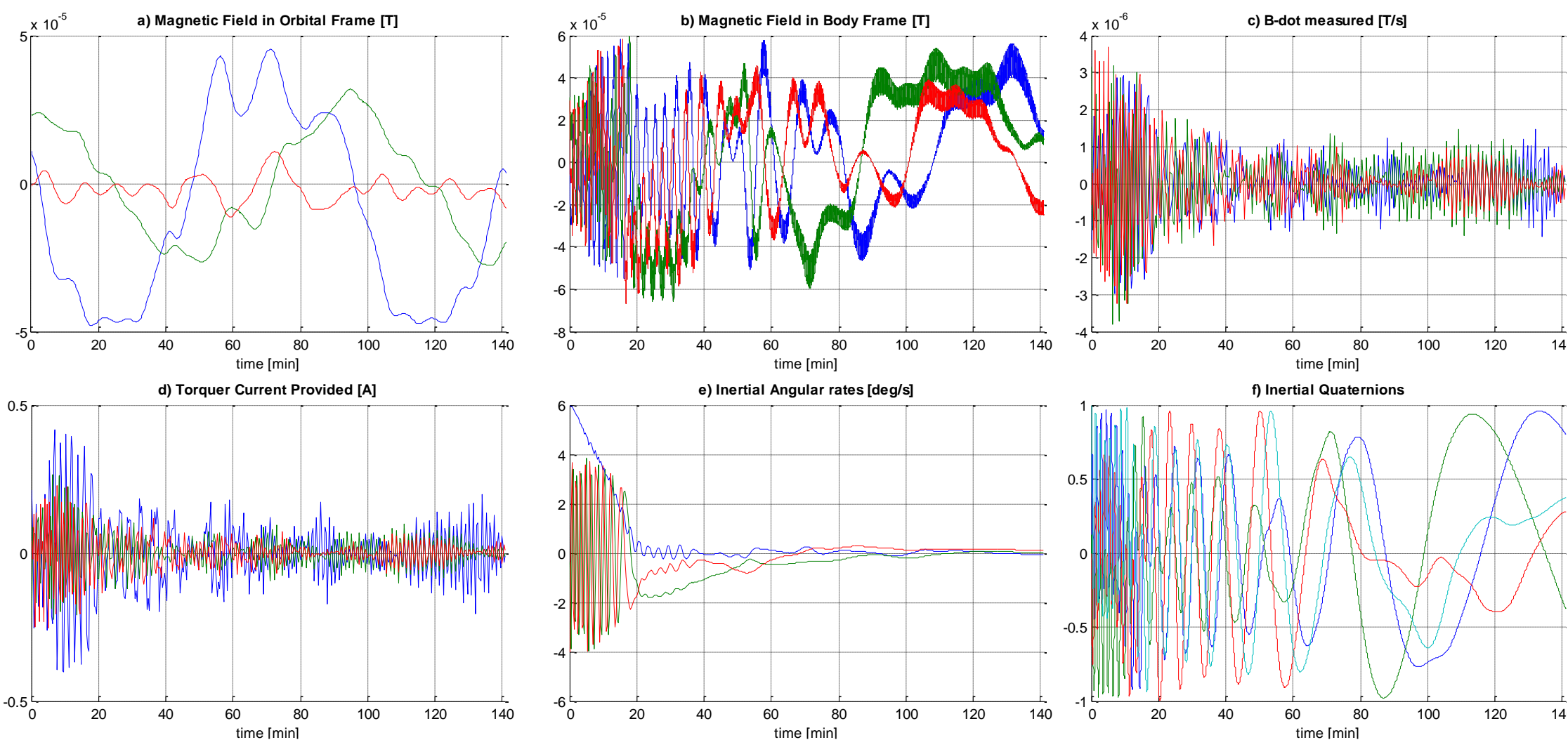

Figure 8.4: Sub-optimal gain performance of the B-dot controller

\begin{tabular}{|c|c|c|}
\hline$K_{b d o t}$ & Pulse Length: $d t[\mathrm{~s}]$ & Final Rate: $\|\omega\|[\mathrm{deg} / \mathrm{s}]$ \\
\hline $\mathbf{3} \times \mathbf{1 0}^{\mathbf{4}}$ & 9.9 & 0.1950 \\
\hline
\end{tabular}




\subsubsection{Local Instability}
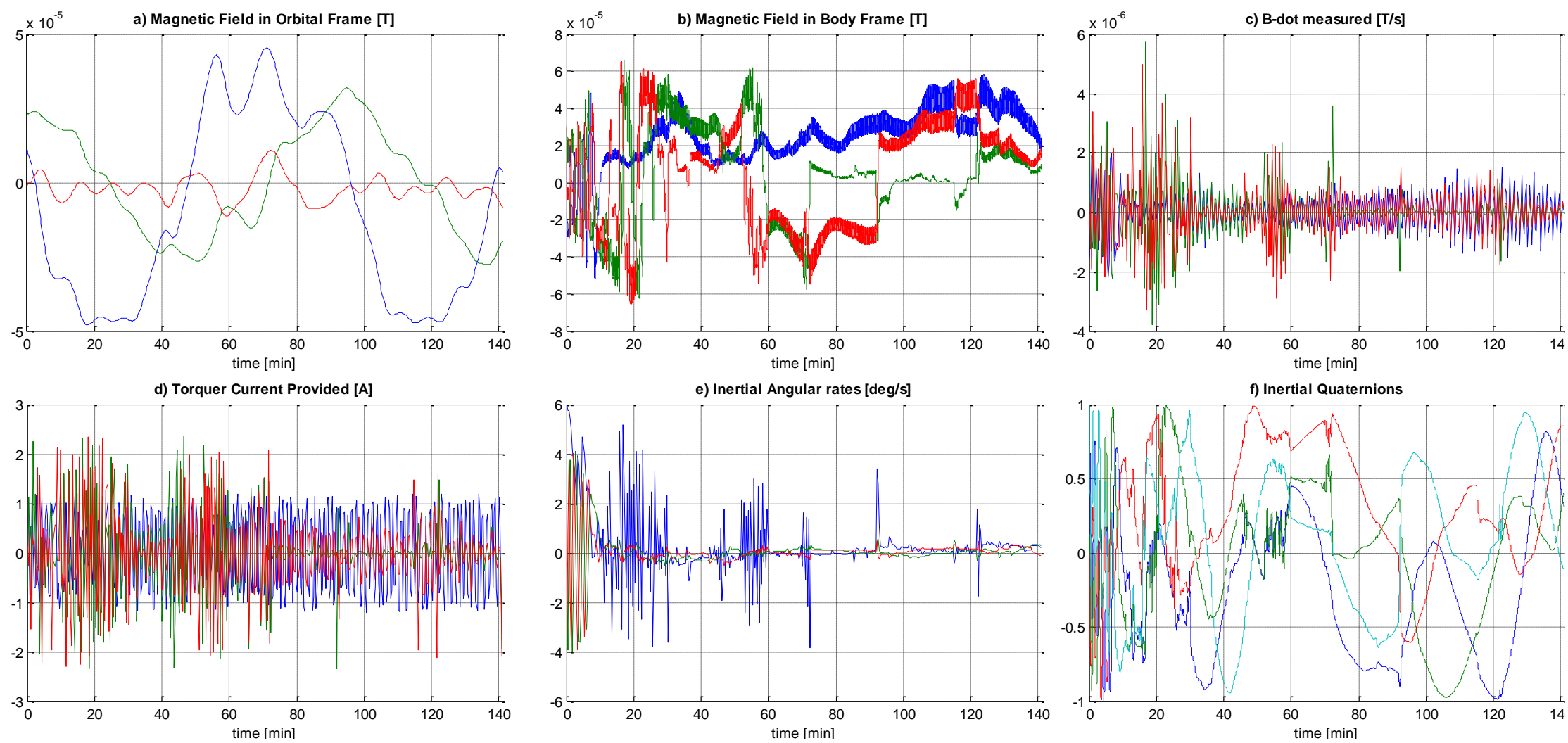

Figure 8.5: Simulation depicting local instabilities of B-dot controller due to improper gain selection

\begin{tabular}{|c|c|c|}
\hline$K_{b d o t}$ & Pulse Length: $d t[\mathrm{~s}]$ & Final Rate: $\|\omega\|[\mathrm{deg} / \mathrm{s}]$ \\
\hline $\mathbf{5} \times \mathbf{1 0}^{\mathbf{7}}$ & 9.9 & 0.4070 \\
\hline
\end{tabular}




\subsubsection{Pulse Time Selection}

While the gain directly impacts the settling time, the pulse time is more directly linked to the stability of the control algorithm. The B-dot controller assumes that the magnetic field remains the same over the period of time $\delta t$ that the measurements are made and remains the same for when the calculated magnetorquer pulse is applied. Increasing the interval makes this assumption less valid and can become unstable when the magnetic field changes too much during the interval or the spacecraft's rotation is high enough that nonlinearity makes the system unstable. However, decreasing the pulse time effectively increases the duty cycle thereby increasing power and computational demands. The pulse times were varied in the simulations given a constant gain selected from the optimal case from the gain selection of $6 \times 10^{4}$. The simulation was run for 1.5 orbits and the final angular rate and total current drawn by the magnetorquers recorded in Figure 8.6.

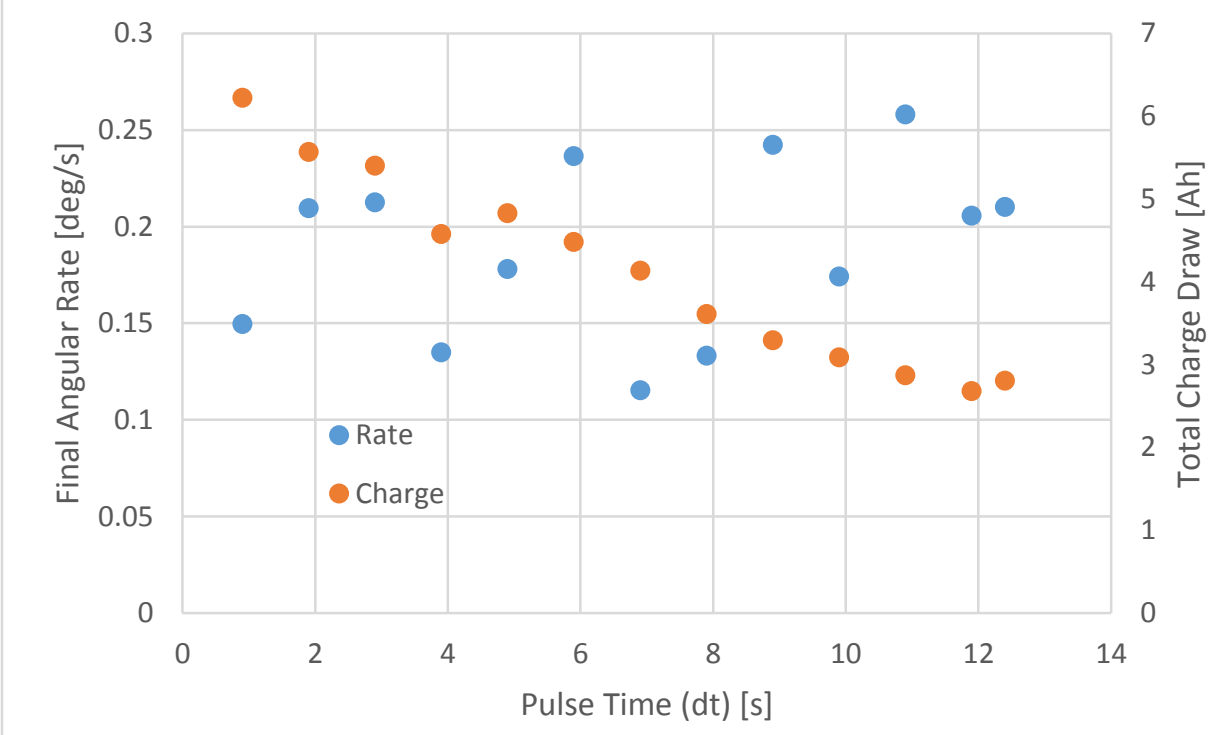

Figure 8.6: B-dot performance for selected range of pulse times 
As can be seen in Figure 8.6, the rate remains relatively consistent at an average of $0.1740 \mathrm{deg} / \mathrm{s}$ for all pulse times until it is increased to 12.5 seconds and the controller diverges. The figure also shows that the current draw from the magnetorquers increases as the pulse time is increased. The ideal pulse time thus remains 9.9 seconds as with the heritage design since it affords a decent margin away from the instability that occurs with longer pulse times but doesn't suffer from the increase in current draw. It should be noted that the ideal pulse time is dependent on the gain and moment of inertia and should be recalculated given a different scenario. The performance of the ideal pulse time has already been shown in the previous section since the ideal pulse time remains the same as the heritage design. Simulation results showing the unstable behavior are shown in the following section. 


\subsubsection{Instability Due to Increased Pulse Time}
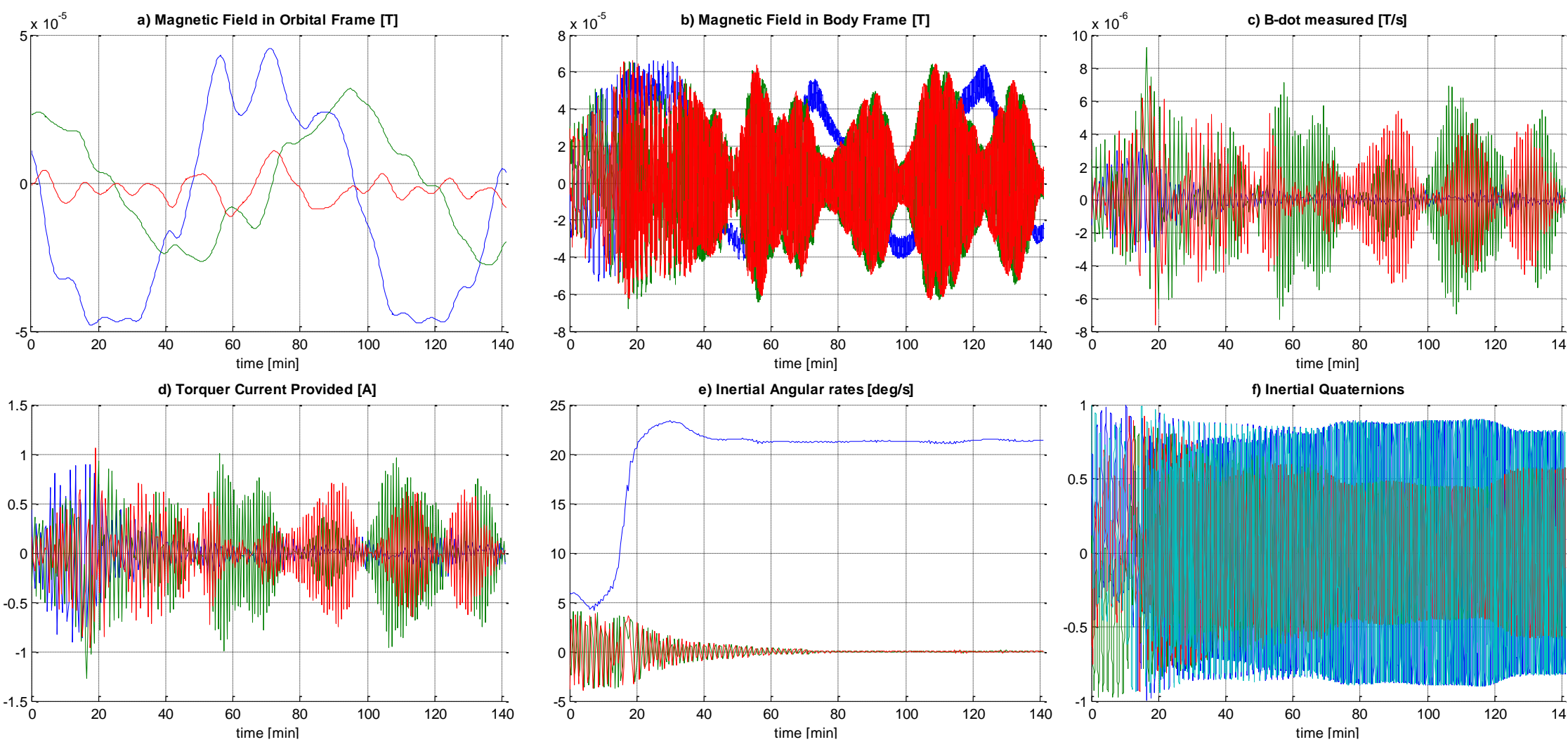

Figure 8.7: Simulation depicting instability of B-dot controller due to increased pulse time

\begin{tabular}{|c|c|c|}
\hline$K_{\text {bdot }}$ & Pulse Length: $d t[\mathrm{~s}]$ & Final Rate: $\|\omega\|[\mathrm{deg} / \mathrm{s}]$ \\
\hline $6 \times 10^{4}$ & $\mathbf{1 2 . 5}$ & 21.3533 \\
\hline
\end{tabular}




\subsubsection{Convergence Criteria Selection}

Proper selection of convergence criteria is crucial as it shuts off the controller at a reasonable final rate to conserve power. Using the optimal gain and pulse time from the previous results, the convergence criteria was varied in the simulations and the final angular rate and total current draw recorded as shown in Figure 8.8.

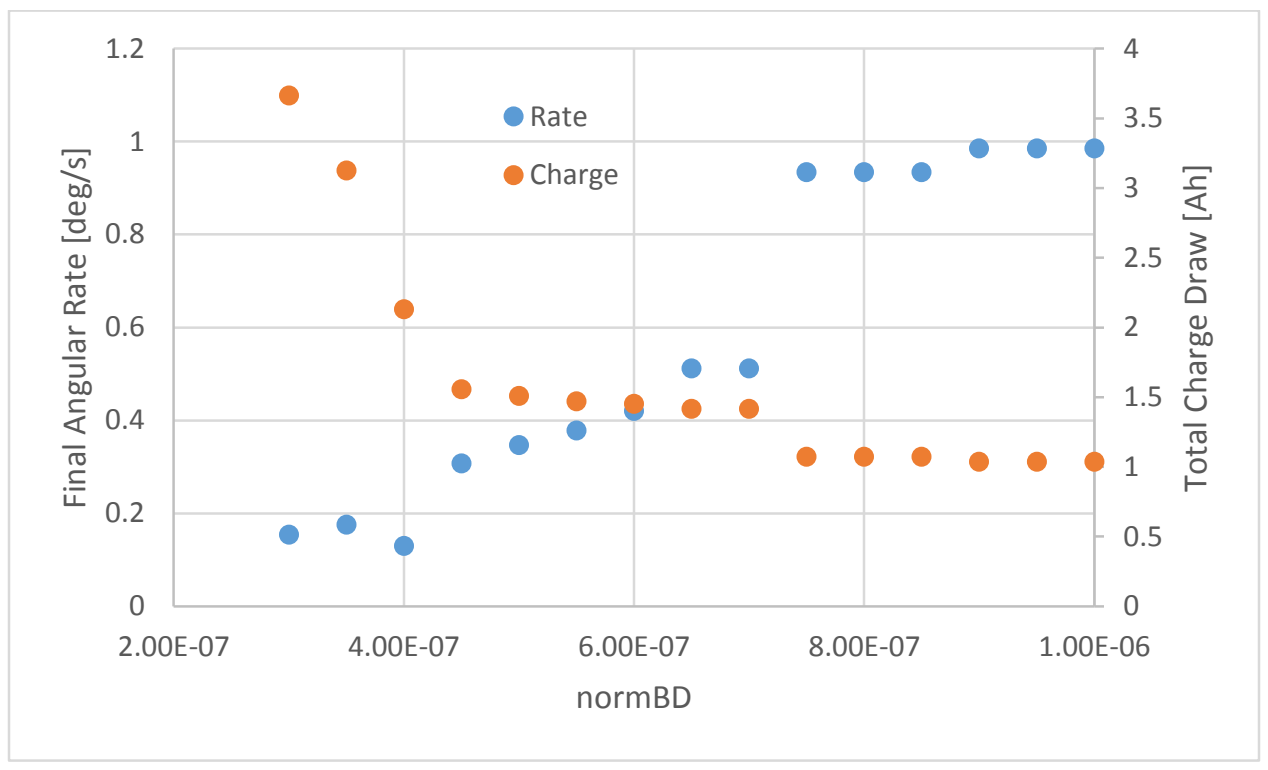

Figure 8.8: B-dot performance for range of selected convergence criteria

As can be seen in Figure 8.8, decreasing the convergence criteria decreased the final angular rate as expected until the limitations of the controller and hardware were reached and the controller wouldn't converge for convergence criteria below $3 \times 10^{-7}$. It can also be seen that decreasing the convergence criteria has diminishing returns in final angular rate at the cost of increasing total current draw. Another interesting result was the large step up in final angular rate that occurred when the convergence criteria was increased beyond $7 \times 10^{-7}$. From the simulations it appears that the B-dot controller is only able to 
perform the initial part of the detumbling with this convergence criteria. Thus the range of convergence criteria for acceptable performance of the B-dot controller is from $3 \times$ $10^{-7}$ to $7 \times 10^{-7}$. The best performance without unnecessary current draw appears to be for a convergence criteria of $4 \times 10^{-7}$.

\subsubsection{Minimum Magnetometer Resolution}

Given the optimum gain and pulse time, the simulations were run with varying magnetometer resolution to find the minimum required resolution for convergence. B-dot was allowed to run for 1.5 orbits and the final angular rates were recorded and can be seen in Figure 8.9.

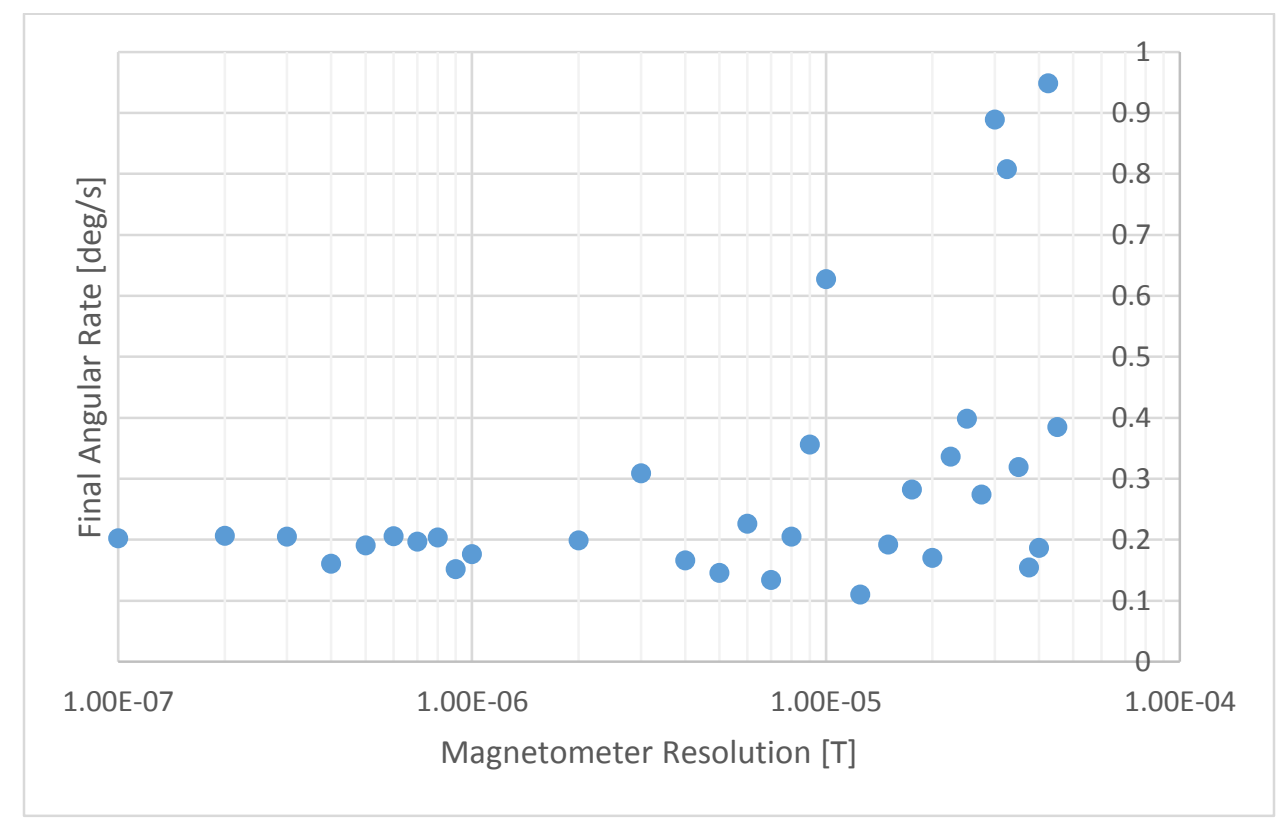

Figure 8.9: B-dot performance for range of magnetometer resolutions

As can be seen in Figure 8.9, the final angular rates for the simulations remain consistent until the resolution of the magnetometers reaches $1 \times 10^{-5}$ Teslas, which is the same 
order of magnitude of the minimum expected Earth magnetic field strength. The impact of the resolution errors become evident in the step-like behavior of the yaw rates in the simulation in the next section. If the resolution is decreased to $5 \times 10^{-5}$ Teslas, the controller diverges because it cannot accurate resolve the Earth magnetic field. Thus the magnetometers should have a resolution on the order of $10^{-6}$ Teslas or better.

Simulations showing the impact of the resolution error and divergence are shown in the following sections. 


\subsubsection{Impact of Resolution Error}
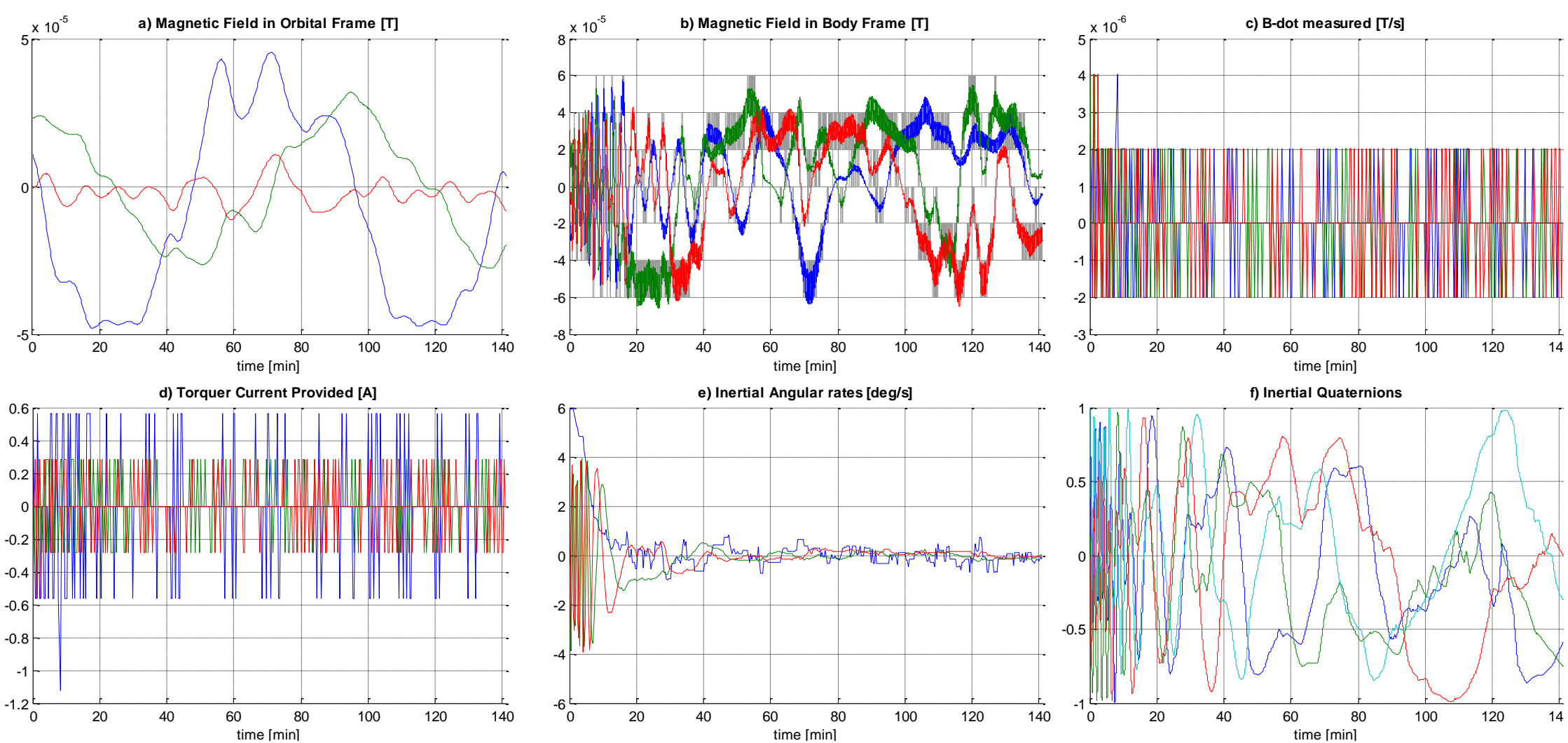

Figure 8.10: Simulation depicting the impact of magnetometer resolution error on the B-dot controller

\begin{tabular}{|c|c|c|c|c|}
\hline$K_{b d o t}$ & $\begin{array}{c}\text { Pulse Length: } \\
d t[\mathrm{~s}]\end{array}$ & $\begin{array}{c}\text { Magnetometer } \\
\text { Noise: } \sigma_{B}[\mathrm{~T}]\end{array}$ & $\begin{array}{c}\text { Magnetometer } \\
\text { Resolution }[\mathrm{T}]\end{array}$ & $\begin{array}{c}\text { Final Rate: } \\
\|\omega\|[\mathrm{deg} / \mathrm{s}]\end{array}$ \\
\hline $6 \times 10^{4}$ & 9.9 & 0 & $\mathbf{2} \times \mathbf{1 0}^{-5}$ & 0.2070 \\
\hline
\end{tabular}




\subsubsection{Divergence due to Resolution Error}
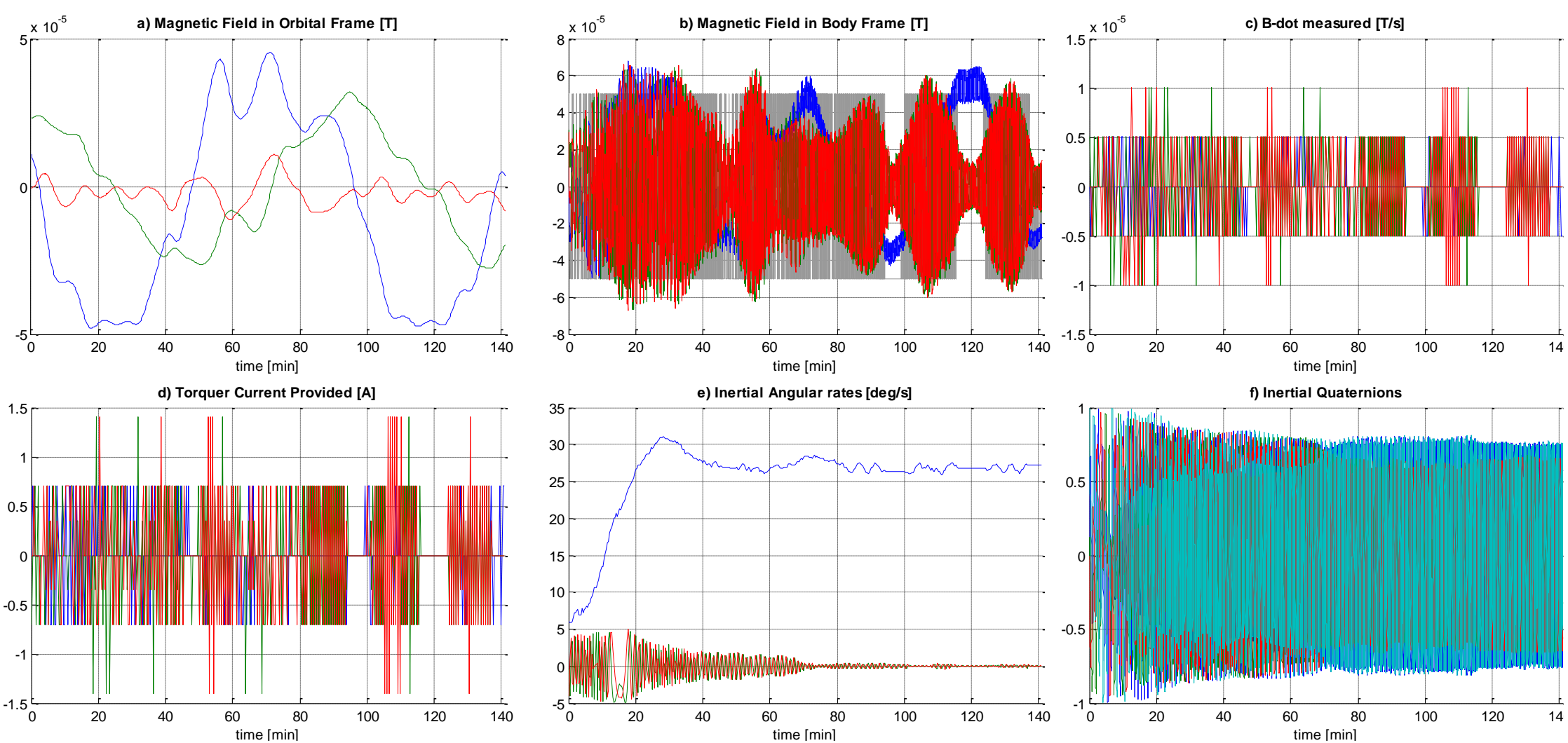

Figure 8.11: Simulation depicting the divergence of the B-dot controller due to magnetometer resolution errors

\begin{tabular}{|c|c|c|c|c|}
\hline$K_{b d o t}$ & $\begin{array}{c}\text { Pulse Length: } \\
d t[\mathrm{~s}]\end{array}$ & $\begin{array}{c}\text { Magnetometer } \\
\text { Noise: } \sigma_{B}[\mathrm{~T}]\end{array}$ & $\begin{array}{c}\text { Magnetometer } \\
\text { Resolution }[\mathrm{T}]\end{array}$ & $\begin{array}{c}\text { Final Rate: } \\
\|\omega\|[\mathrm{rad} / \mathrm{s}]\end{array}$ \\
\hline $6 \times 10^{4}$ & 9.9 & 0 & $\mathbf{5} \times \mathbf{1 0}^{-5}$ & 27.2826 \\
\hline
\end{tabular}




\subsubsection{Magnetometer Noise Tolerance}

Given the optimum gain and pulse time, the simulations were run with a magnetometer resolution of $1 \times 10^{-5}$ Tesla and the standard deviation of the magnetometer noise varied to find the maximum allowable noise for convergence. B-dot was allowed to run for 1.5 orbits and the final angular rates were recorded and can be seen in Figure 8.12.

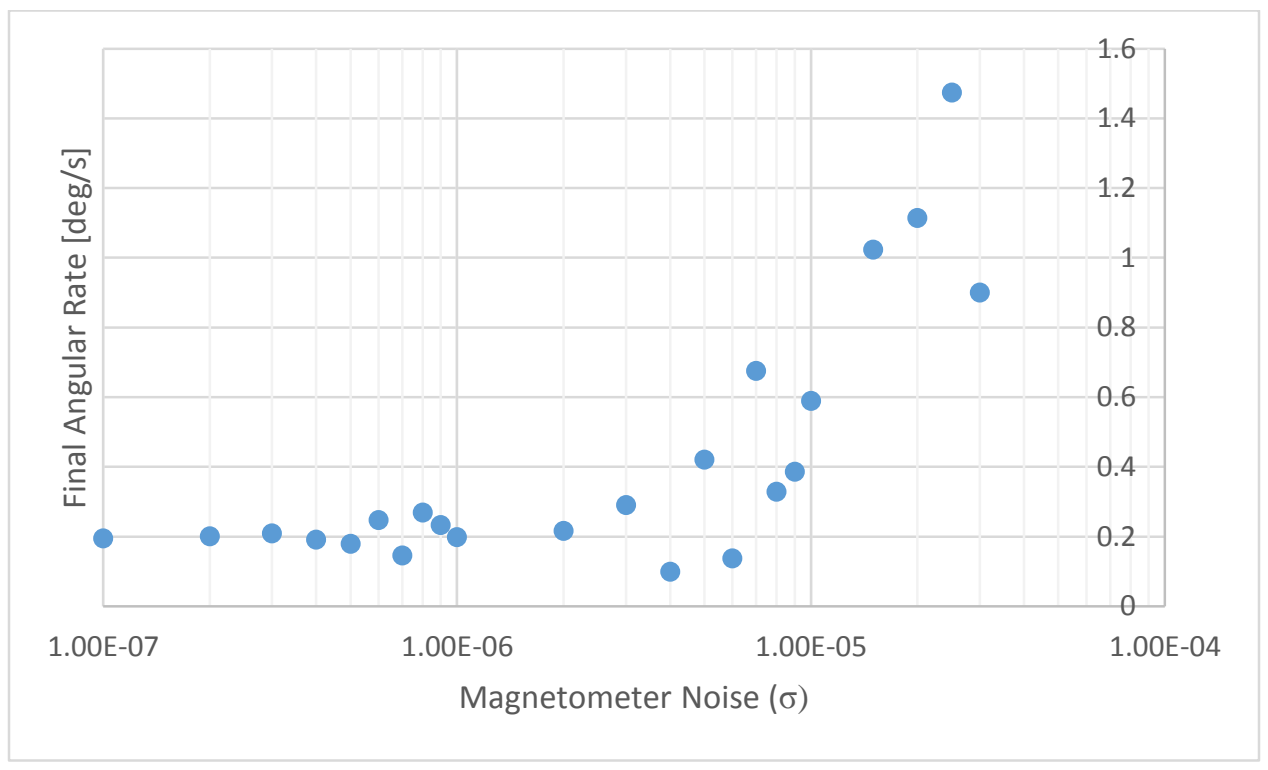

Figure 8.12: B-dot performance for range of magnetometer noise levels

As can be seen in Figure 8.12, the angular rate remains consistent until the magnetometer noise has a standard deviation around $3 \times 10^{-6}$, about an order of magnitude less than the minimum magnetic field strength. The final angular rates of the simulation increase to over $1 \mathrm{deg} / \mathrm{s}$ as the standard deviation of the noise is increased to $3 \times 10^{-5}$, the same magnitude as the Earth magnetic field, after which the controller diverges. 


\subsection{Guide to PD Controller Results Plots}

In the following sections, several key simulations have been selected from the many run to demonstrate various aspects of the performance of the PD controller. The results of each simulation are summarized in a single figure containing six subplots a-f which are detailed in Table 8.3. The $\mathrm{x}$-axis of all subplots are given in minutes. The line of each subplot except for the quaternions are colored accordingly as

$$
X_{\text {Body }} \text { :Blue } \quad Y_{\text {Body }}: \text { Green } \quad Z_{\text {Body }}: \text { Red }
$$

For the quaternion plots the vector components are represented by the above colors and approximate the body orientation when close to alignment with the reference frame. The scalar component of the quaternion is represented by the cyan colored line.

The line types also represent different information as follows:

- Solid: Actual values used by the simulation representing the most accurate information available.

- Dashed: Desired values used by the control algorithm

- Gray: Measured values used by the control algorithm that include resolution and noise errors. 
Table 8.3: Summary of PD controller results plots

\section{a) Quaternion Error}

- Shows the quaternion relating the actual and desired orientation of the satellite.

- The vector components of the error quaternion are used to calculate the control torque

- Gray lines represent the error quaternion calculated using the measured bodyinertial quaternion and includes any resolution or noise errors

\section{d) Pointing Error}

- Represents the error rotation angle about the quaternion error axis.

- Calculated from the scalar component of the error quaternion as follows:

$$
\theta_{\text {err }}=2 \operatorname{acos}\left(q_{4, \text { err }}\right)
$$

\section{b) Inertial Angular Rates}

- The components of the actual simulated values of the body-inertial rates

- The dotted lines represent the desired inertial rates

- Gray lines represent the measured values and include any resolution or noise errors

- The difference between the measured body-inertial and desired rates is used in the control law

\section{e) Orbital Angular Rates}

The components of the actual simulated values of the body-orbital rates

- $\quad$ The dotted lines represent the desired orbital rates

- This plot is included as reference and is not used in the calculation of the control torques

\section{c) Inertial Quaternions}

- The components of the actual simulated values of the body-inertial quaternion

- The dotted lines represent the desired inertial quaternion

- Gray lines represent the measured values and include any resolution or noise errors

- The measured and desired quaternions are used to calculate the quaternion error in subplot a.

\section{f) Orbital Quaternions}

- The components of the actual simulated values of the body-orbital quaternion

- The dotted lines represent the desired orbital quaternion

- This plot is included as reference and is not used in the calculation of the control torques. 


\subsection{Initial Attitude Acquisition}

After successfully detumbling, the spacecraft will have relatively low angular rates that can be conservatively approximated as:

$$
\omega_{0}=\left[\begin{array}{lll}
0.005 & -0.005 & 0.002
\end{array}\right]
$$

Although B-dot will reduce the angular rates of the spacecraft, the orientation will be unknown and possibly at a large angle from the desired ram pointing orientation. For a robust test of the PD controller's performance, the initial orientation is set to 120 degrees off of the target orientation as given by the quaternion

$$
q_{0}=\left[\begin{array}{llll}
0.5 & 0.5 & 0.5 & 0.5
\end{array}\right]
$$

As with the detumbling simulations, a polar orbit with an inclination of 95 degrees at an altitude of $475 \mathrm{~km}$ was selected for the simulation. A conservative estimate of the 3U's deployed moment of inertia that was used in the simulation is

$$
I_{\text {total }}=\left[\begin{array}{ccc}
0.0049 & 0 & 0 \\
0 & 0.1236 & 0 \\
0 & 0 & 0.1238
\end{array}\right]
$$

The given deployed moment of inertia is gravity gradient stable per the smelt parameters defined previously as indicated by the red $\mathrm{x}$ in the Lagrange stability region seen in Figure 8.13. 


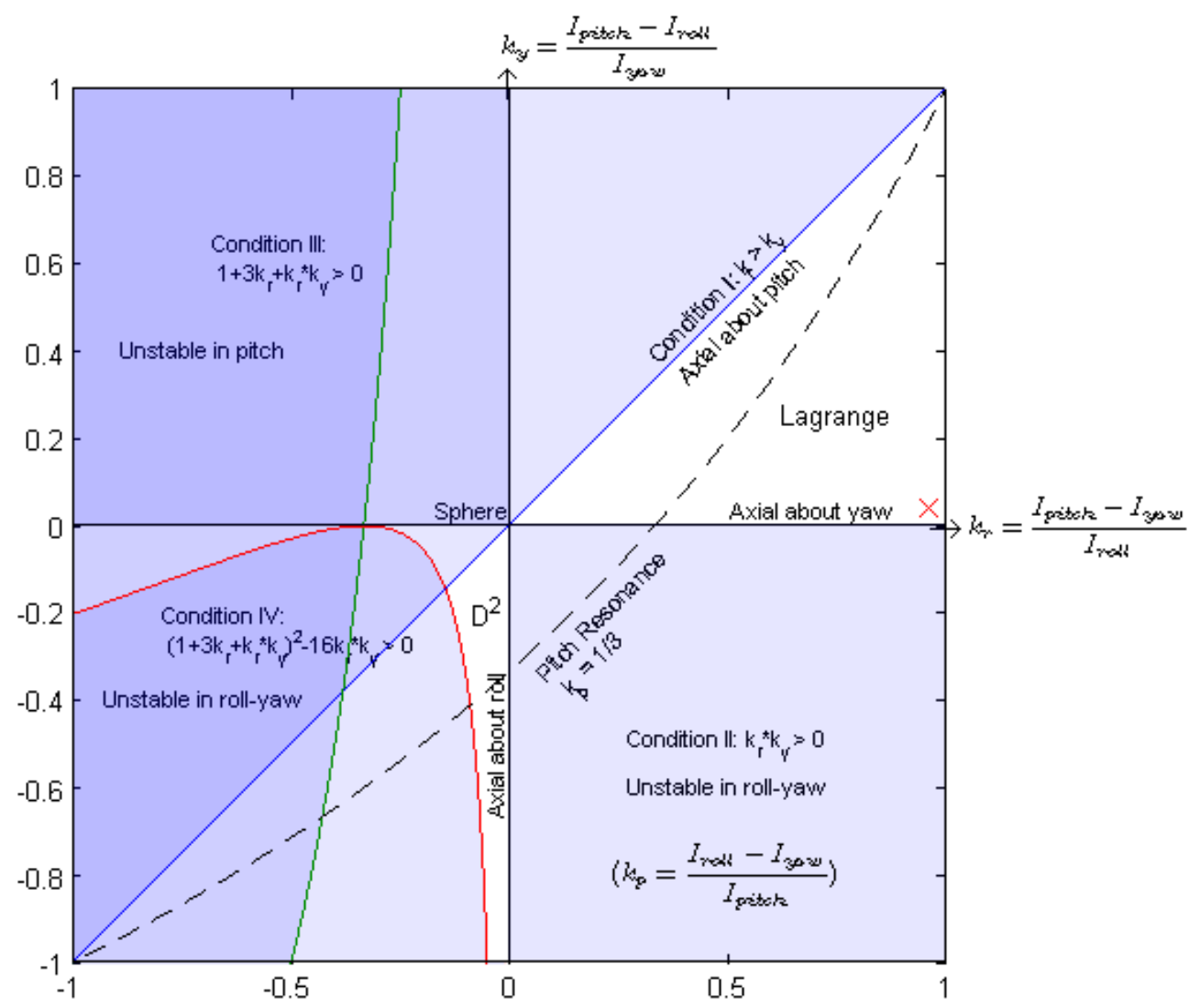

Figure 8.13: Smelt parameter plane with deployed configuration plotted (indicated by red $\mathrm{X}$ )

\subsubsection{Gain Selection}

As discussed previously, Wie et al. shows that global stability for the quaternion feedback regulator is guaranteed provided that the rate gain is scaled by the spacecraft moments of inertia (Wie, Weiss and Arapostathis). When the control torques are applied directly as if the system had three reaction wheels, the controller responds well for a wide range of gains scaled by the moment of inertia. However, when direct actuation is replaced by the use of magnetorquers, the yaw motion exhibits local instabilities for the wide range of gains tested. This makes sense given that the gain design technique outlined by Wie et al. is based on a second order linear differential equation and active 
magnetic control is inherently non-linear due to the inability to actuate in all 3 axes at all times.

By eliminating the scaling by the moment of inertia for the rate gain, the yaw instabilities were reduced but the system still didn't converge. Next the gyroscopic cancelation component was removed and the yaw instability issues improved and the system converged for a narrow range of gains. Upon applying the current limiters that are a necessity of the magnetorquer hardware, the range of gains that converge narrows further. The current limiters essentially requires lower gain values in order to avoid the instability that comes with clipping the control torques.

Ultimately, a line search method was adopted to find the optimum gains. First a battery of equal rate and orientation gains were simulated that were scaled so that the rate gain was three orders of magnitude higher than the orientation gain. Given the results of the first battery of tests, the pair of gains with the fastest convergence time was selected and the rate gain varied while the orientation gain was held constant. Given the results of the second battery of tests, the pair of gains with the fastest convergence time was selected and then the orientation gain was varied while the rate gain was held constant. This procedure was repeated until minimal improvement was seen thus suggesting an optimal set of gains have been found. The data from this line search can be found in appendix B and the results from the optimal set of gains seen in the following section. Also included is a simulation with a sub-optimal set of gains to show the corresponding losses in performance. 


\subsubsection{Optimal Case}
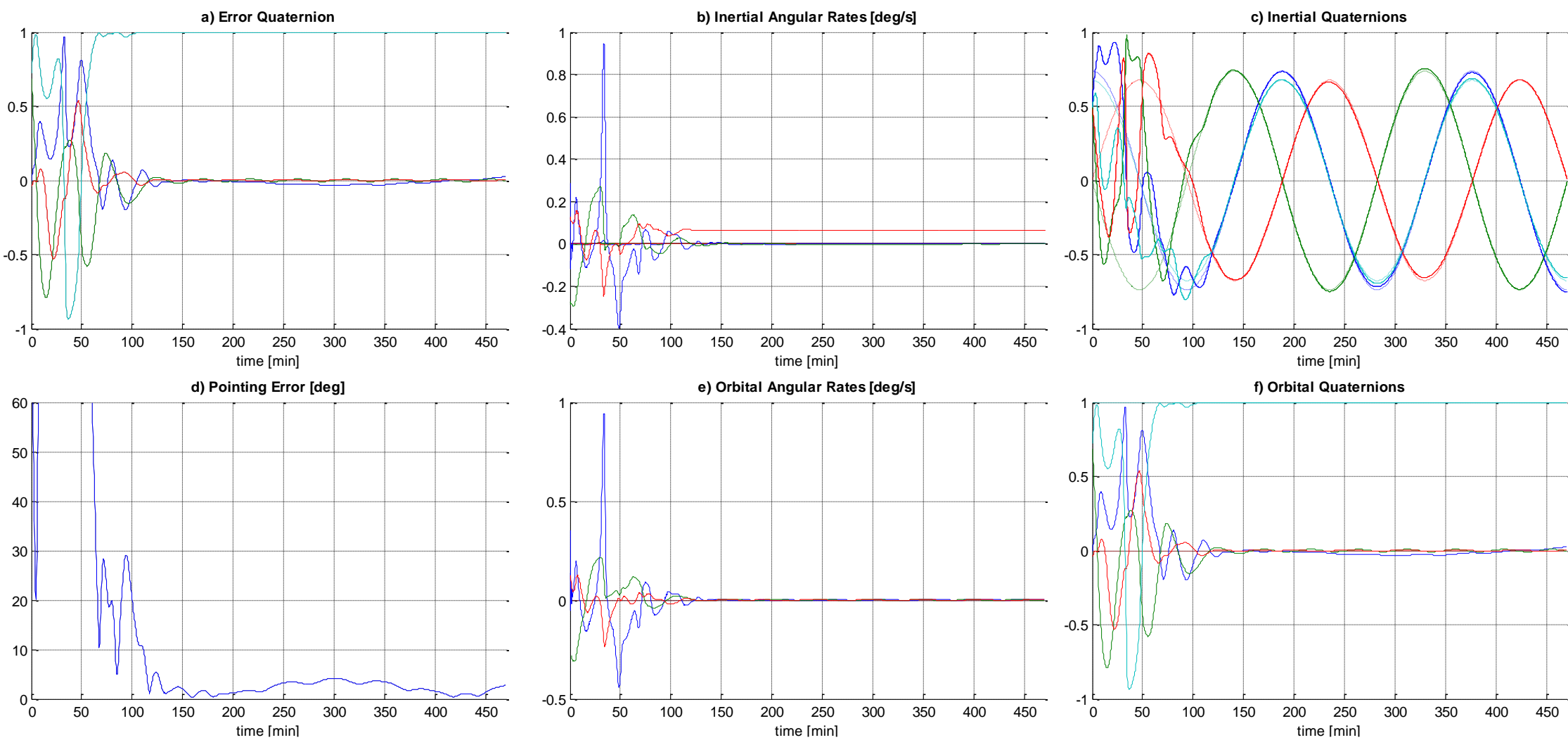

Figure 8.14: Optimal gain performance of PD controller for initial acquisition of the target orientation

\begin{tabular}{|c|c|c|c|c|c|}
\hline$D_{P D}$ & $K_{P D}$ & $\begin{array}{c}\text { Convergence Criteria: } \\
\omega_{t o l}[\mathrm{deg} / \mathrm{s}], \angle_{\text {tol }}[\mathrm{deg}]\end{array}$ & $\begin{array}{c}\text { Time to Converge } \\
{[\mathrm{min}]}\end{array}$ & $\begin{array}{c}\text { Rate: }\|\omega\| \text { Pointing Error } \\
{[\mathrm{deg}]}\end{array}$ \\
\hline $8 \times 10^{-4}$ & $5 \times 10^{-6}$ & $1 \times 10^{-3}, 2$ & 197.165 & $5.74 \times 10^{-4}$ & 1.1883 \\
\hline
\end{tabular}




\subsubsection{Sub-Optimal Case}
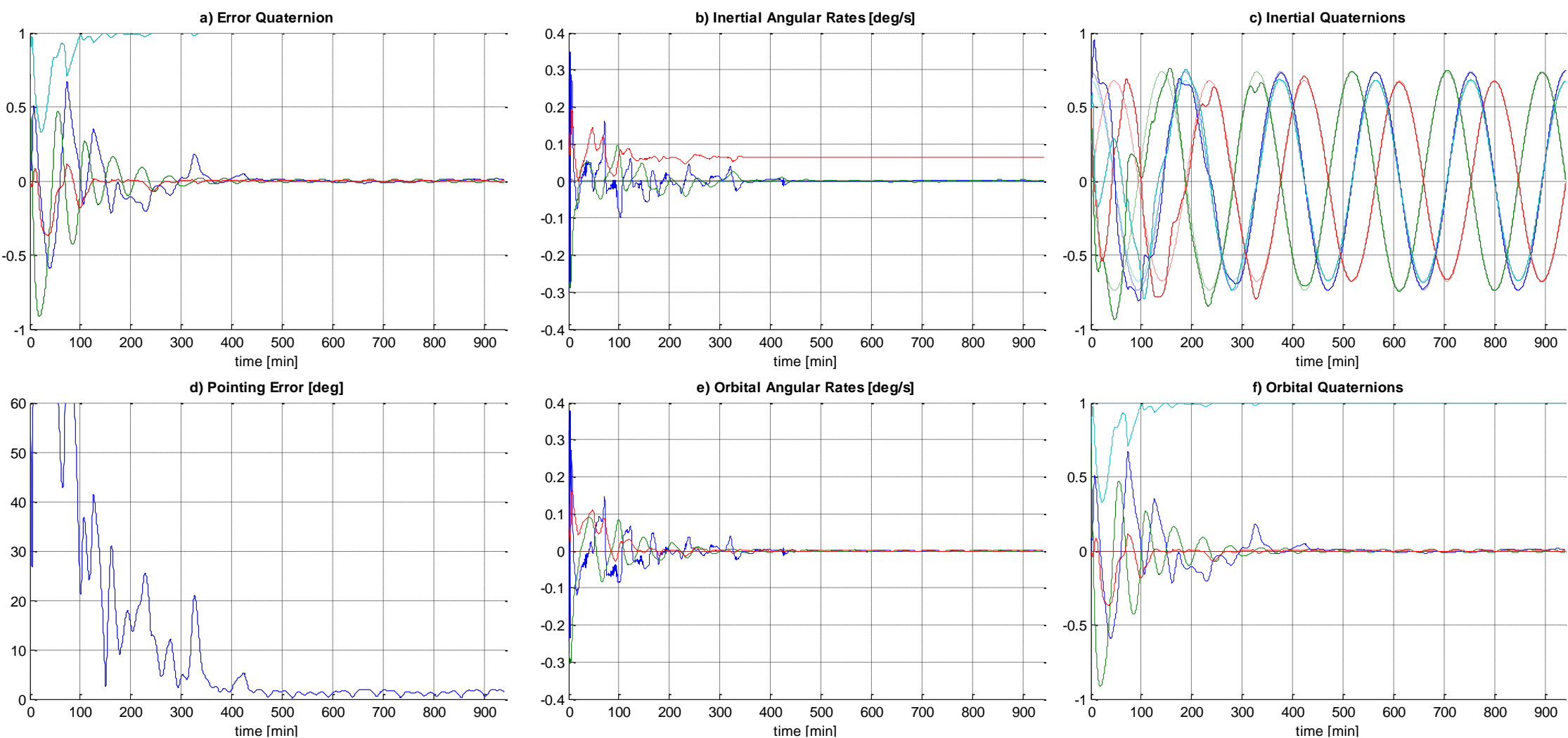

Figure 8.15: Sub-optimal gain performance of PD controller for initial acquisition of the target orientation

\begin{tabular}{|c|c|c|c|c|c|}
\hline$D_{P D}$ & $K_{P D}$ & $\begin{array}{c}\text { Convergence Criteria: } \\
\omega_{t o l}[\mathrm{deg} / \mathrm{s}], \angle_{\text {tol }}[\mathrm{deg}]\end{array}$ & $\begin{array}{c}\text { Time to Converge } \\
{[\mathrm{min}]}\end{array}$ & $\begin{array}{c}\text { Rate: }\|\omega\| \text { Pointing Error } \\
{[\mathrm{deg}]}\end{array}$ \\
\hline $1 \times 10^{-3}$ & $1 \times 10^{-6}$ & $1 \times 10^{-3}, 2$ & 450.165 & $9.45 \times 10^{-4}$ & 1.8455 \\
\hline
\end{tabular}




\subsubsection{Pulse Time Selection}

To determine the impact of pulse time on the performance of the three axis controller, the optimal gains selected from the previous section were used while the pulse time was varied. The time to converge and charge used remained relatively constant at an average of 300 minutes and 1.33 Amp-hours for a large range of pulse times as can be seen in Figure 8.16

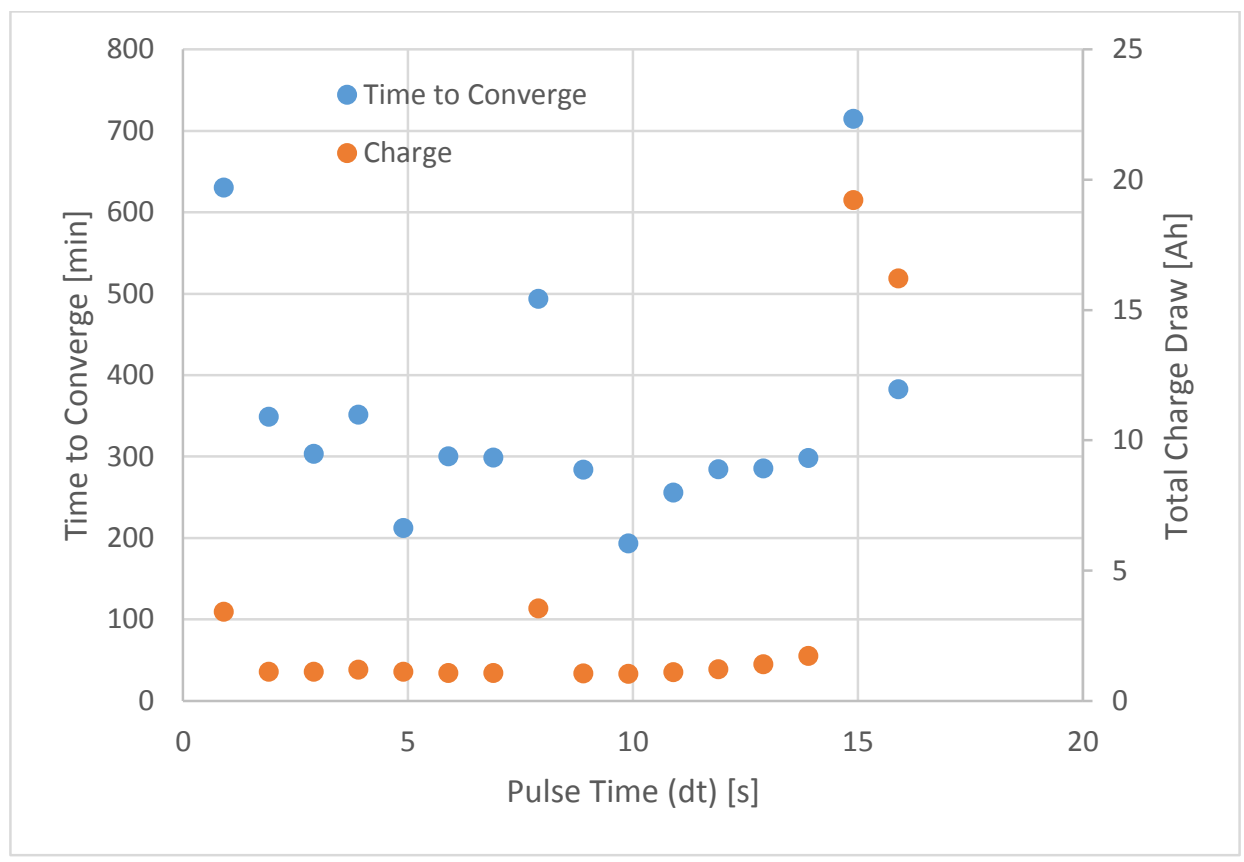

Figure 8.16: PD controller performance for range of pulse times

Once the pulse times were increased to13.9 seconds and greater, localized instability was observed coinciding with passing over the magnetic poles. As with the B-dot controller, increased pulse times cause the performance of the controller to suffer near the poles due to the fact that the magnetic field vector that the pulse was calculated for changes too much during the pulse. Decreased pulse times lead to increases in computational 
demands and must be balanced with the stability of the controller. In order maintain a comfortable margin from the instabilities observed with increased pulse time without overtaxing the flight computer, a pulse time of 9.9 seconds was selected and is consistent with the B-dot controller design.

\subsubsection{Convergence Criteria}

Selection of the convergence criteria for the PD controller directly impacts the final pointing accuracy of the spacecraft. The offset in spacecraft orientation from the target orientation and the angular rate at convergence will determine the offset and oscillation of the spacecraft once the momentum wheel has been brought to the desired speed. The impact of the offset in pointing was found to have far less of an impact than the angular rates and thus the convergence criteria for offset was conservatively set at 2 degrees. The following figure shows the behavior of the satellite in the target orientation given different angular rates without the influence of aerodynamic and solar radiation pressure torques. 


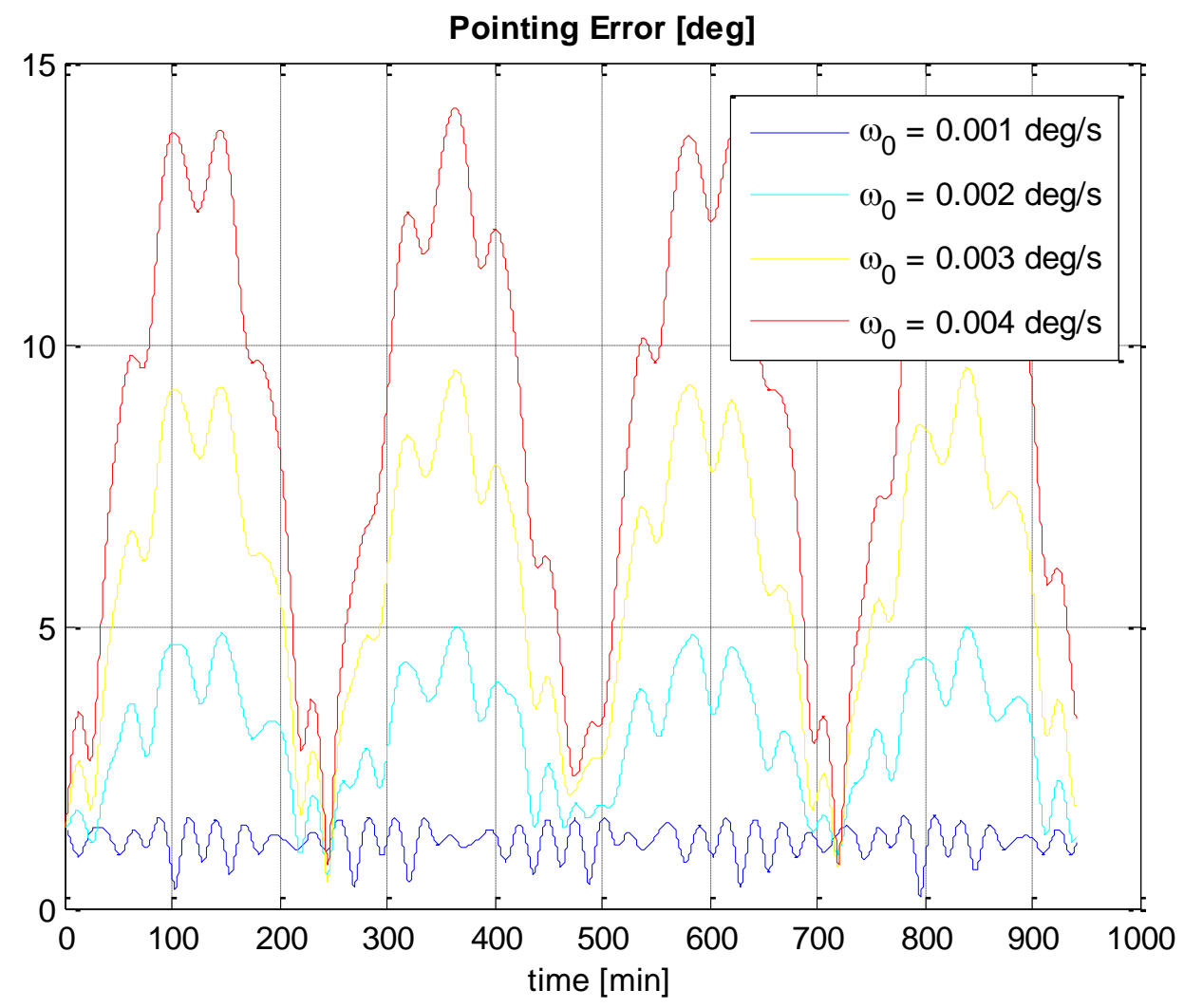

Figure 8.17: Spacecraft pointing error for various initial angular rates

As can be seen in Figure 8.17, angular rates above $0.003 \mathrm{deg} / \mathrm{s}$ cause the satellite to oscillate outside of the required ten degree pointing. In order to ensure that the momentum wheel spins up in an acceptable orientation and that the spacecraft will remain in the target orientation barring a large offset in the center of gravity, a convergence criteria of $0.001 \mathrm{deg} / \mathrm{s}$ was set.

\subsubsection{Orientation Error Tolerance}

Given the optimal gains and pulse time, the simulations were run while varying the resolution of the attitude quaternion to find the minimum required resolution for convergence. Since the vector portion of the quaternion error approaches zero as the 
controller converges, the impact of the rounding errors isn't seen until after the controller has already brought the spacecraft close to the target orientation. As a result, the controller will converge with a minimum resolution of $1 \times 10^{-1}$ as can be seen in the next section. However, the attitude knowledge errors exceed 40 degrees which is outside of the required \pm 5 degree pointing knowledge requirement required by the payload. Having a resolution of at least $1 \times 10^{-5}$ guaranteed no impact on the performance of the controller.

Given a resolution of $1 \times 10^{-5}$, noise was introduced into the attitude quaternions with varying standard deviations to find the maximum allowable amount of noise for convergence. As with the resolution, the noise primarily effects the performance of the controller after it gets close to the target orientation. It was found that the controller still converged with attitude quaternion noise with a standard deviation of $5 \times 10^{-2}$ as can be seen in the following section. However, this noise represented errors in pointing knowledge as high as 60 degrees and is thus unacceptable per the payload pointing knowledge requirements. It is recommended that the noise not exceed the resolution of $1 \times 10^{-5}$ in order to avoid an impact on controller performance. 


\subsubsection{Minimum Resolution}
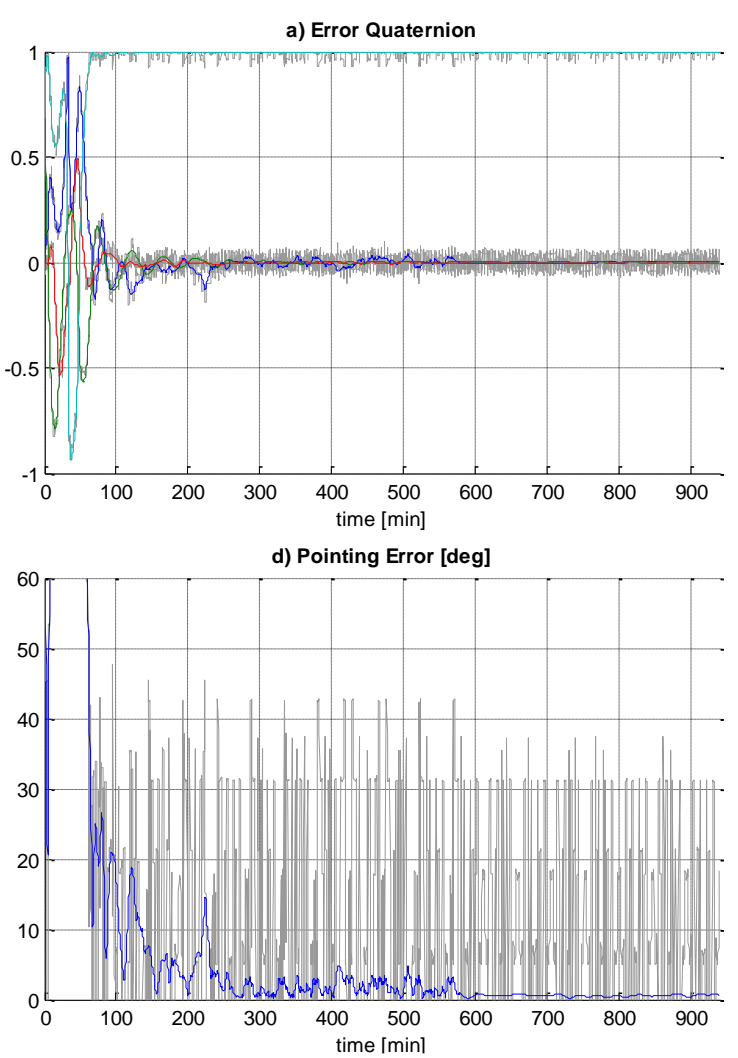

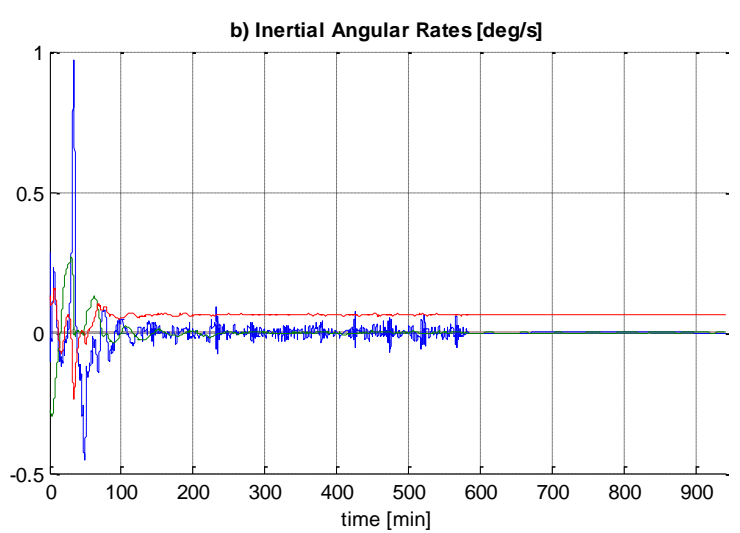

e) Orbital Angular Rates [deg/s]

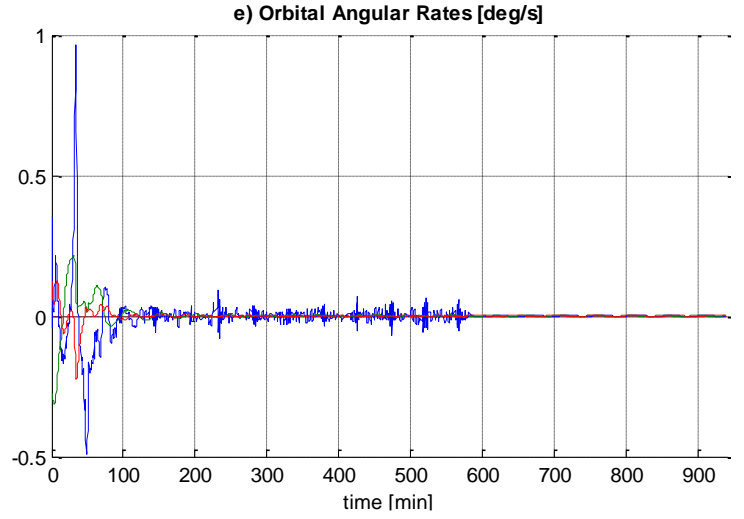

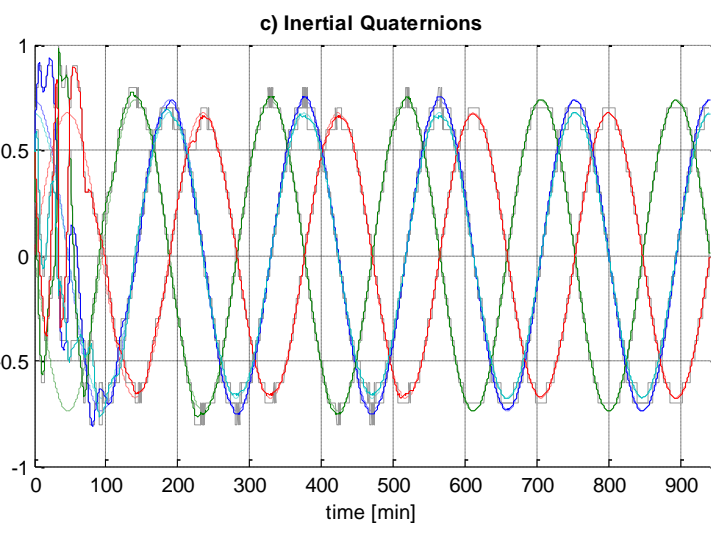

f) Orbital Quaternions

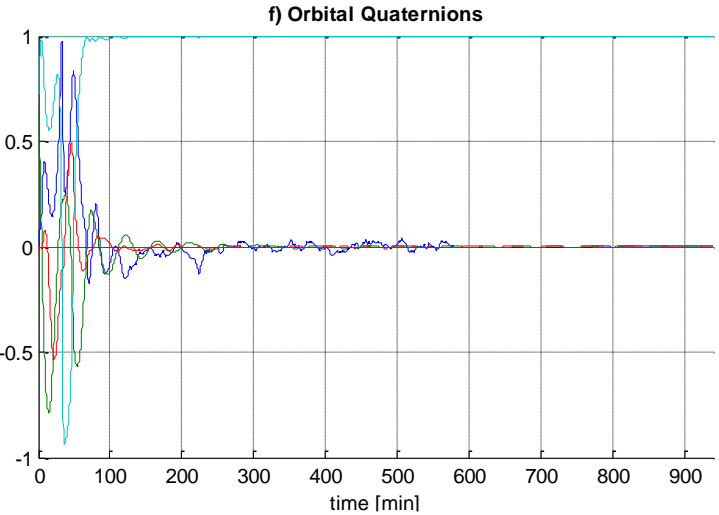

Figure 8.18: Simulation depicting the impact of quaternion resolution on the PD controller

\begin{tabular}{|c|c|c|c|c|c|}
\hline$D_{P D}$ & $K_{P D}$ & $\begin{array}{c}\text { Convergence Criteria: } \\
\omega_{\text {tol }}[\mathrm{deg} / \mathrm{s}], \angle_{\text {tol }}[\mathrm{deg}]\end{array}$ & $\begin{array}{c}\text { Time to Converge } \\
{[\mathrm{min}]}\end{array}$ & $\begin{array}{c}\text { Rate: }\|\omega\| \text { Pointing Error } \\
{[\mathrm{deg}]}\end{array}$ \\
\hline $8 \times 10^{-4}$ & $5 \times 10^{-6}$ & $1 \times 10^{-3}, 2$ & 582.4983 & $6.7008 \times 10^{-4}$ & 0.5558 \\
\hline
\end{tabular}




\subsubsection{Maximum Noise}
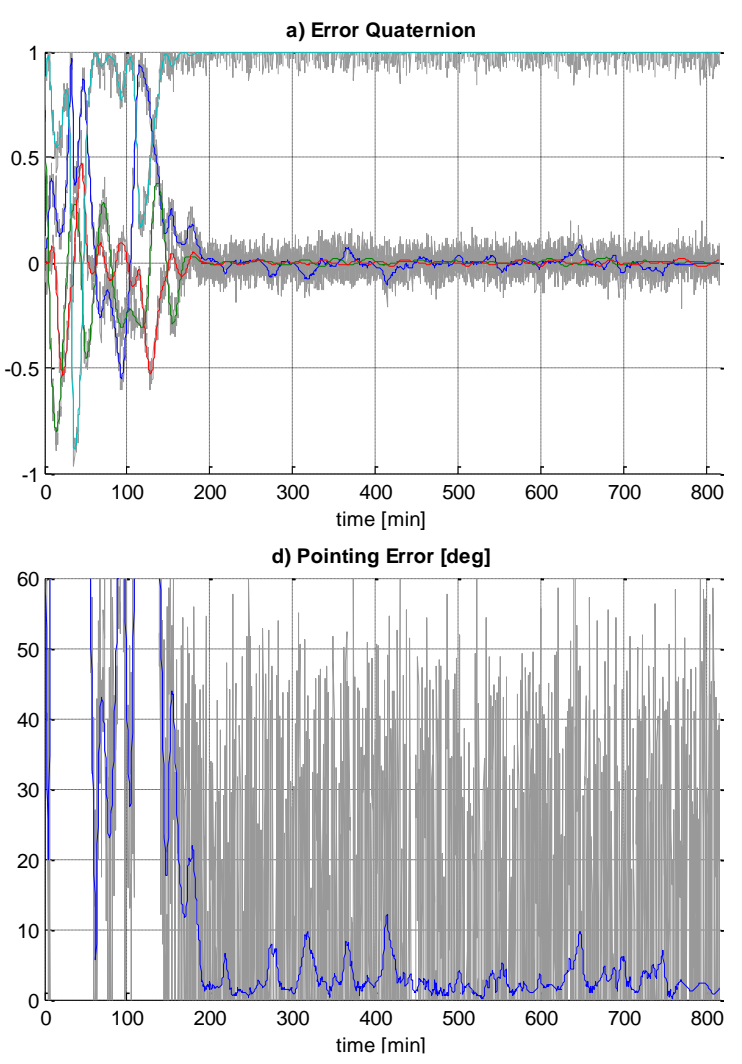

b) Inertial Angular Rates [deg/s]

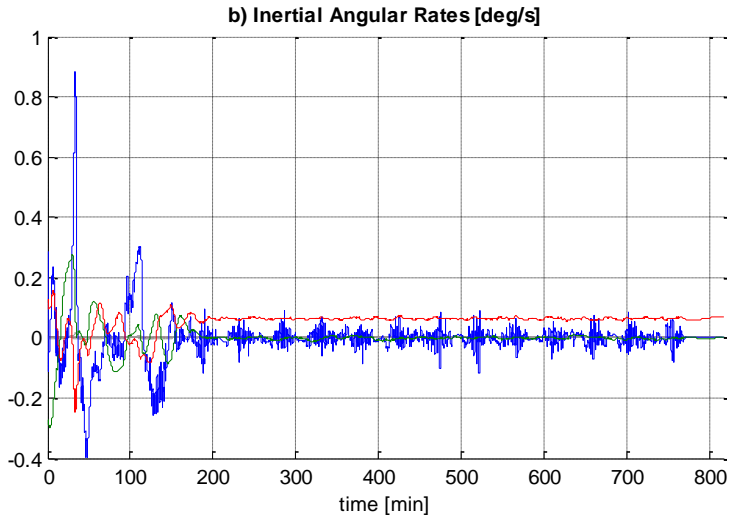

e) Orbital Angular Rates [deg/s]

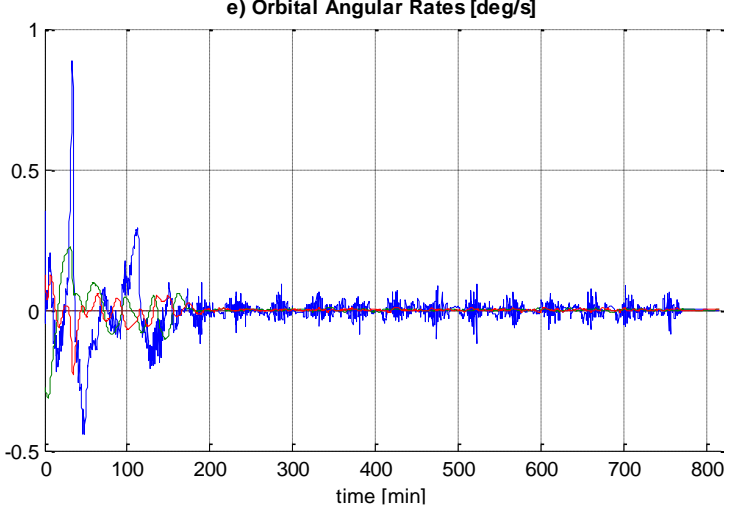

c) Inertial Quaternions

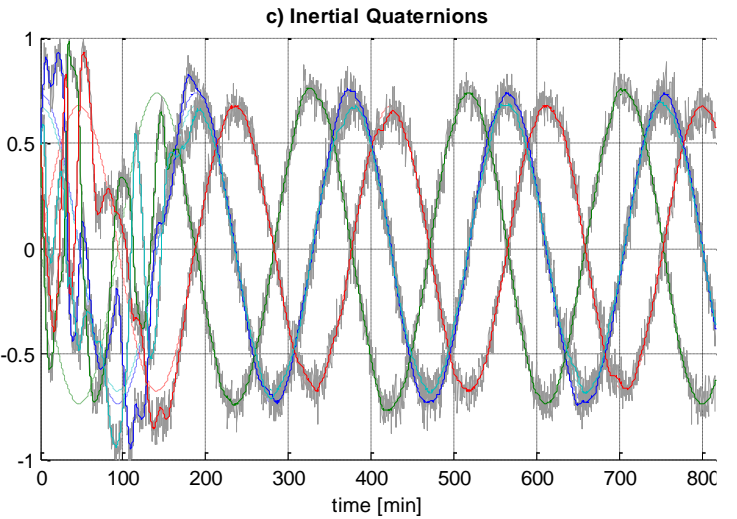

f) Orbital Quaternions

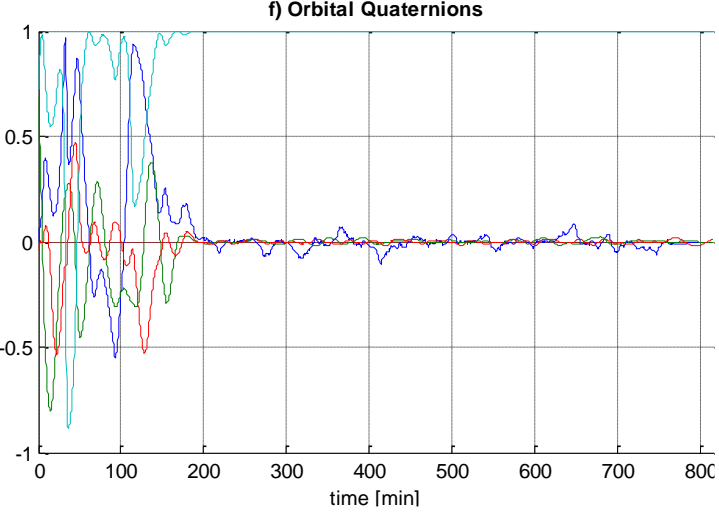

Figure 8.19: Simulation depicting the impact of quaternion noise on the PD controller

\begin{tabular}{|c|c|c|c|c|c|}
\hline$D_{P D}$ & $K_{P D}$ & $\begin{array}{c}\text { Convergence Criteria: } \\
\omega_{\text {tol }}[\mathrm{deg} / \mathrm{s}], L_{\text {tol }}[\mathrm{deg}]\end{array}$ & $\begin{array}{c}\text { Time to Converge } \\
{[\mathrm{min}]}\end{array}$ & $\begin{array}{c}\text { Rate: }\|\omega\|[\mathrm{deg} / \mathrm{s}] \\
\text { Pointing Error } \\
{[\mathrm{deg}]}\end{array}$ \\
\hline $8 \times 10^{-4}$ & $5 \times 10^{-6}$ & $1 \times 10^{-3}, 2$ & 769.8317 & $9.69 \times 10^{-4}$ & 2.7363 \\
\hline
\end{tabular}




\subsubsection{Angular Rate Error Tolerance}

Given the optimal gains and pulse time, the simulations were run while varying the resolution of the angular rates to find the minimum required resolution for convergence. As with the previous findings, the impact of the rounding errors isn't seen until the controller is approaching convergence and the rates approach zero. Thus the controller will converge with a minimum resolution of $1 \times 10^{-4} \mathrm{rad} / \mathrm{s}\left(5.73 \times 10^{-3} \mathrm{deg} / \mathrm{s}\right)$ as can be seen in the next section. However, the lack of resolution allowed the controller to converge at too high of an angular rate resulting in undesirably large oscillations. It is recommended to have a resolution of at least $1 \times 10^{-7} \mathrm{rad} / \mathrm{s}\left(5.73 \times 10^{-6} \mathrm{deg} / \mathrm{s}\right)$ to guarantee that it will not impact the performance of the controller.

Given a resolution of $1 \times 10^{-7} \mathrm{rad} / \mathrm{s}$, the noise was introduced into the angular rates with varying standard deviations to find the maximum allowable amount of noise for convergence. As with the resolution, the impact on performance due to the noise is primarily as the rates approach zero as the spacecraft approaches its target orientation. The controller will still converge with angular rate noise with a standard deviation of $1 \times$ $10^{-4} \mathrm{rad} / \mathrm{s}\left(5.73 \times 10^{-3} \mathrm{deg} / \mathrm{s}\right)$ as seen in the next section. However, this level of noise allowed the controller to converge at too high of an angular rate resulting in undesirably large oscillations. It is therefore recommended that the angular rate error not exceed $1 \times$ $10^{-5} \mathrm{rad} / \mathrm{s}\left(5.73 \times 10^{-4} \mathrm{deg} / \mathrm{s}\right)$. 


\subsubsection{Minimum Resolution}
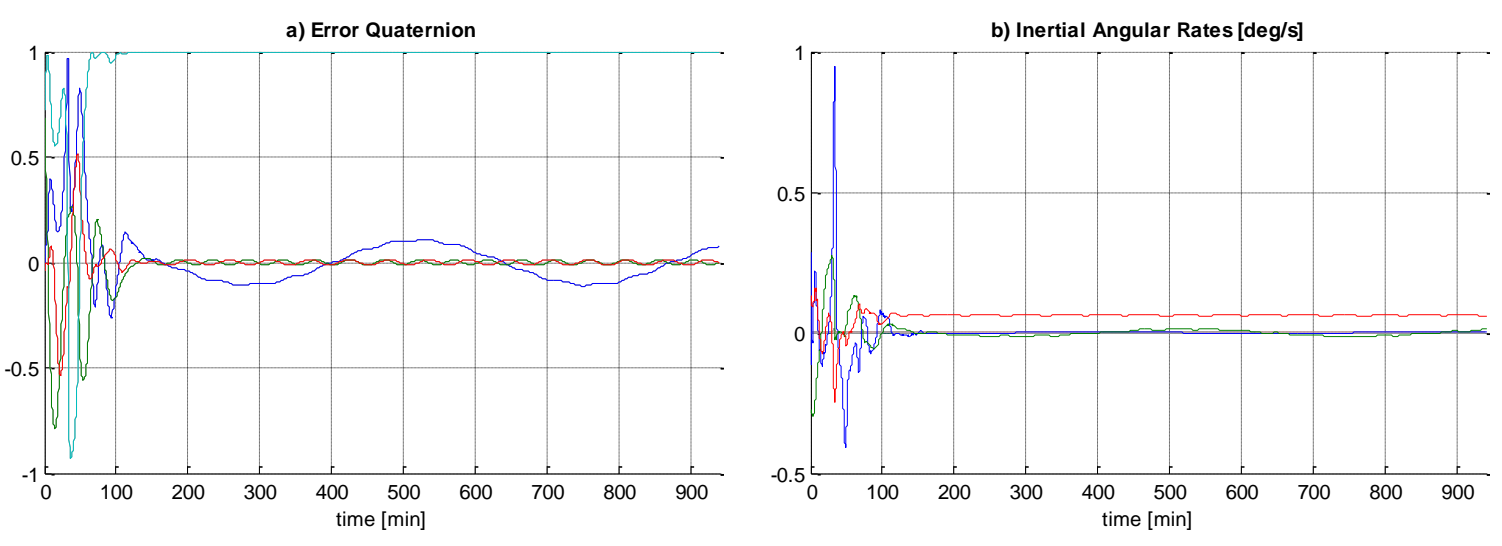

Pointing Error [deg]
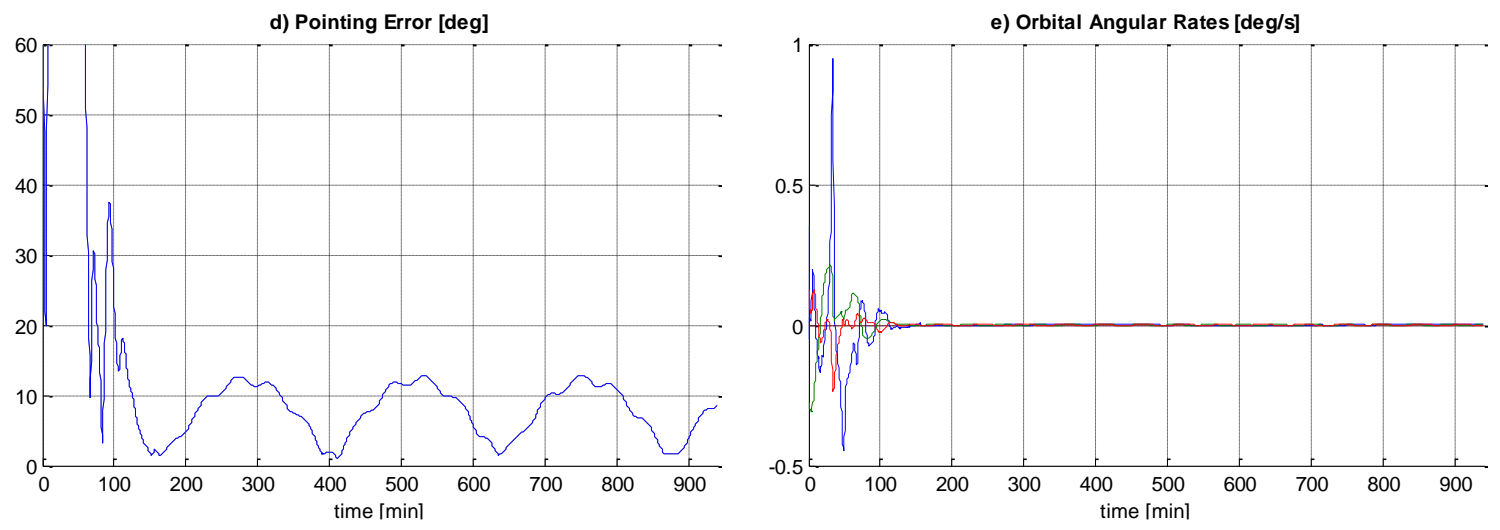

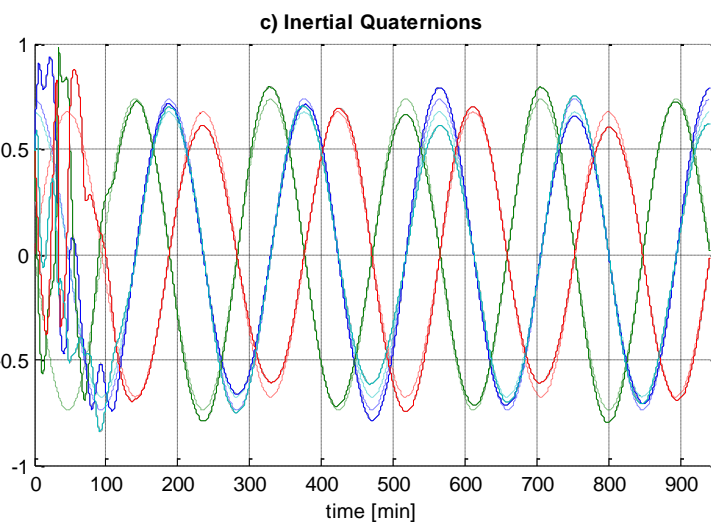

f) Orbital Quaternions

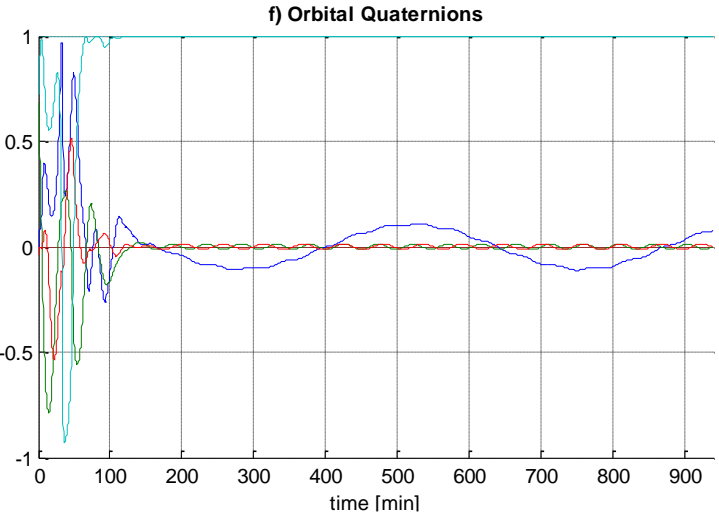

Figure 8.20: Simulation depicting the impact of rate resolution on the PD controller

\begin{tabular}{|c|c|c|c|c|c|}
\hline$D_{P D}$ & $K_{P D}$ & $\begin{array}{c}\text { Convergence Criteria: } \\
\omega_{\text {tol }}[\mathrm{deg} / \mathrm{s}], \angle_{\text {tol }}[\mathrm{deg}]\end{array}$ & $\begin{array}{c}\text { Time to Converge } \\
{[\mathrm{min}]}\end{array}$ & $\begin{array}{c}\text { Rate: }\|\omega\| \text { Pointing Error } \\
{[\mathrm{deg}]}\end{array}$ \\
\hline $8 \times 10^{-4}$ & $5 \times 10^{-6}$ & $1 \times 10^{-3}, 2$ & 159.8317 & 0.0039 & 1.8298 \\
\hline
\end{tabular}




\subsubsection{Maximum Noise}

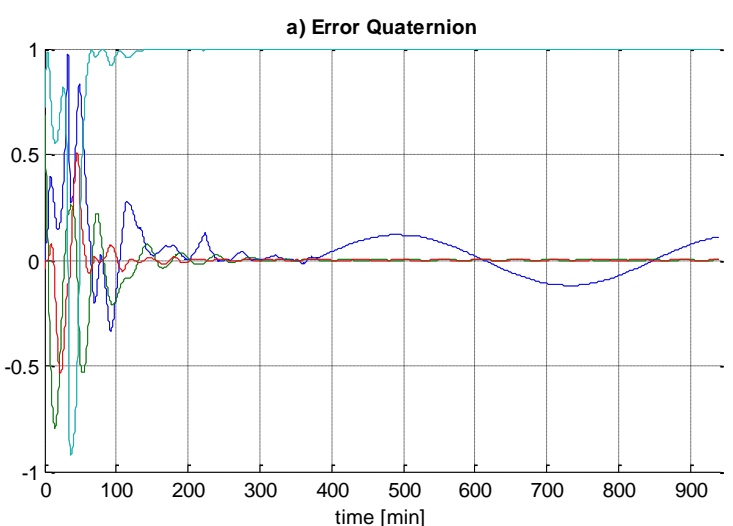

d) Pointing Error [deg]

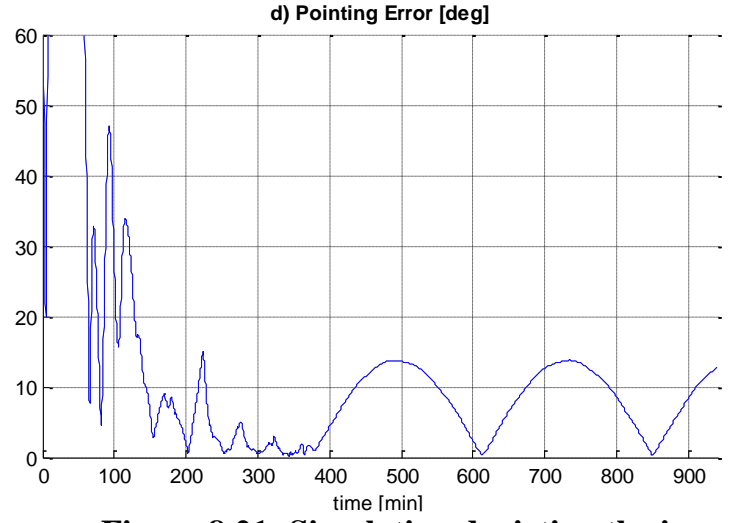

b) Inertial Angular Rates [deg/s]

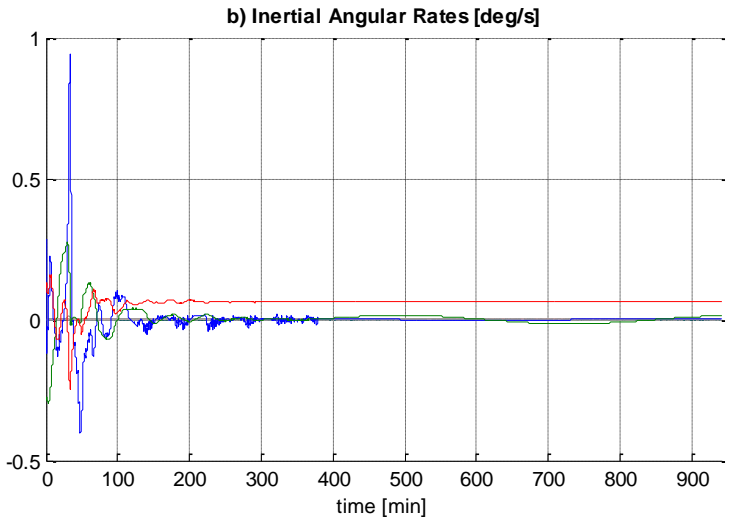

e) Orbital Angular Rates [deg/s]

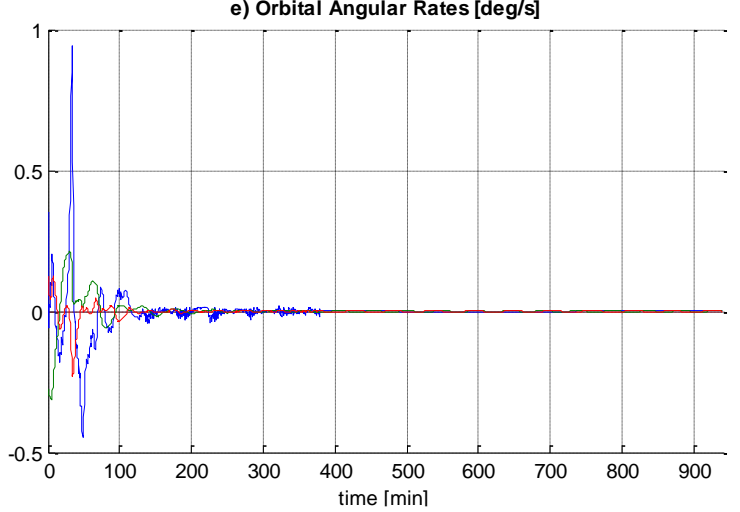

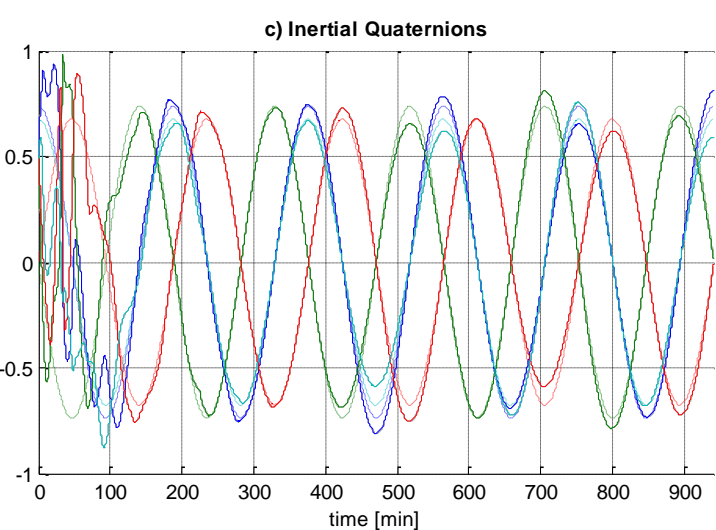

f) Orbital Quaternions

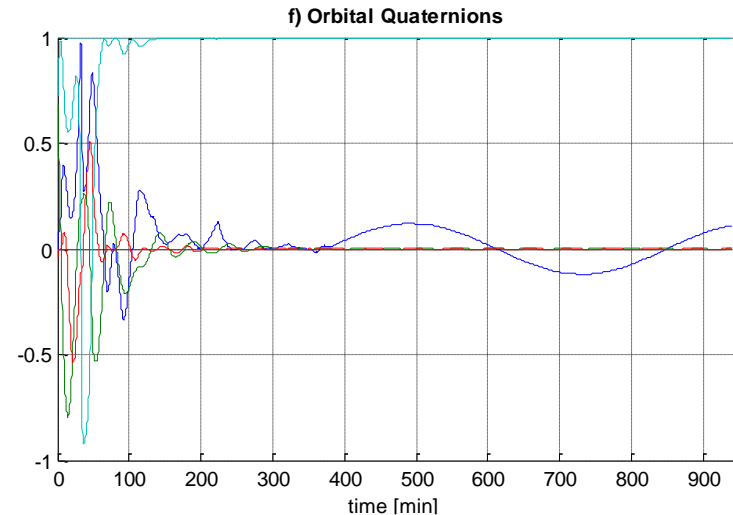

Figure 8.21: Simulation depicting the impact of rate noise on the PD controller

\begin{tabular}{|c|c|c|c|c|}
\hline$D_{P D}$ & $K_{P D}$ & $\begin{array}{c}\text { Convergence Criteria: } \\
\omega_{t o l}[\mathrm{deg} / \mathrm{s}], \angle_{\text {tol }}[\mathrm{deg}]\end{array}$ & $\begin{array}{c}\text { Time to Converge } \\
{[\mathrm{min}]}\end{array}$ & $\begin{array}{c}\text { Rate: }\|\omega\| \text { Pointing Error } \\
{[\mathrm{deg}]}\end{array}$ \\
\hline $8 \times 10^{-4}$ & $5 \times 10^{-6}$ & $1 \times 10^{-3}, 2$ & 379.8317 & 0.8953 \\
\hline
\end{tabular}




\subsection{Wheel Spin Up}

After successfully acquiring the target ram-pointing orientation, the spacecraft's orientation and angular rates can be conservatively set as

$$
\begin{aligned}
& \omega_{0}=\left[\begin{array}{llll}
-1.9685 \times 10^{-6}-1.7453 \times 10^{-5} & 0.0011
\end{array}\right] \\
& q_{0}=\left[\begin{array}{llll}
0.7372 & -5.3829 \times 10^{-4} & 0.0123 & 0.6756
\end{array}\right]
\end{aligned}
$$

Which corresponds to pointing errors of 1 degree in the pitch and yaw axes and bodyorbital angular rates of $0.001 \mathrm{deg} / \mathrm{s}$ which are consistent with the convergence criteria established previously. The simulations were run for ten orbits with the wheel initially spinning up with the PD controller on and the convergence check off until the wheel was at speed and the controller allowed to correct any offset in pointing created during spin up. Initial simulations led to several key adjustments to the controller and the definition of the constant torque at which the wheel would be commanded to track until it reached the desired speed.

From the initial battery of simulations, it was discovered that including the gyroscopic cancelation component of controller was detrimental to performance and created instabilities due to the changes in wheel speed. It was also found that the gyroscopic cancelation component overpowered the relatively small control torques required for the fine adjustments after the wheel was at speed. Thus the gyroscopic cancelation component was omitted from the controller after the initial acquisition. It was also discovered that the optimal gains from the initial acquisition needed to be adjusted by having the rate gain reduced from $8 \times 10^{-4}$ to $5 \times 10^{-4}$ in order to avoid the controller 
overreacting to changes in the rate error when the spacecraft is already within the desired orientation. The next design task was to set the desired angular speed of the momentum wheel and the constant torque at which it would be brought to this desired speed.

The Sinclair wheel selected is capable of spinning at a maximum of $3410 \mathrm{rpm}$ resulting in a momentum storage of $10 \mathrm{mNm} / \mathrm{s}$ drawing $0.16 \mathrm{~W}$ at steady state (Sinclair Data sheet). As a compromise between momentum storage and power consumption, a nominal angular rate of $2000 \mathrm{rpm}$ was selected achieving $5.9 \mathrm{mNm} / \mathrm{s}$ momentum storage at a steady state power consumption of just $0.1 \mathrm{~W}$. As mentioned previously, the momentum wheel will be commanded to track a constant torque target during spin up until the desired angular speed is reached. Spin up of the wheel will inevitably cause a temporary offset in pointing in the pitch axis as a result of a constant torque being applied in one direction and being opposed by the gravity gradient torques and magnetorquers. The constant torque in the spacecraft pitch axis was selected to be low enough to be opposed primarily by the gravity gradient torques and produce an offset that remained within the pointing requirement of \pm 10 degrees. By commanding the wheel to spin up by tracking a constant torque of $2 \times 10^{-7} \mathrm{Nm}$, the spacecraft maintained a 7 degree offset in pointing on the pitch axis and quickly reacquires the desired orientation once the wheel reached $2000 \mathrm{rpm}$ as can be seen in the following section. As mentioned previously, the torque control on the wheel has noise on the order of $10^{-6} \mathrm{Nm}$ associated with it. Despite the noise level being greater than the commanded torque, the controller can still track the commanded torque. The spacecraft still remains within 10 degrees of the ram direction 
but noise in the torque controller causes the offset angle during spin up to vary between 3 and 9 degrees as can be seen in the next section. 


\subsubsection{Wheel Spin Up Performance}
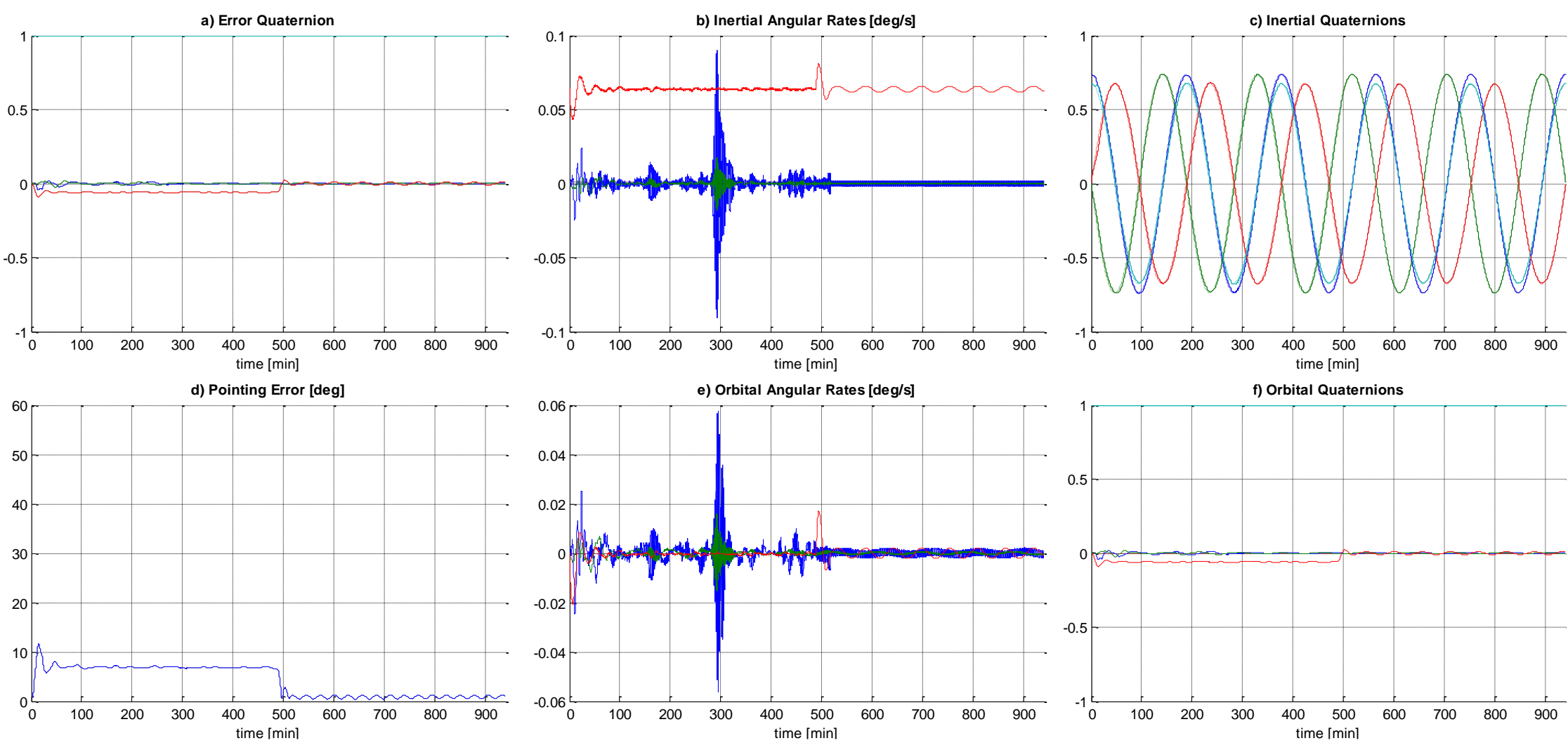

Figure 8.22: PD controller performance during wheel spin up

\begin{tabular}{|c|c|c|c|c|c|c|c|}
\hline$D_{P D}$ & $K_{P D}$ & $\begin{array}{c}\text { Final Wheel } \\
\text { Speed [RPM] }\end{array}$ & $\begin{array}{c}\text { Spin Up } \\
\text { Torque [Nm] }\end{array}$ & $\begin{array}{c}\text { Convergence Criteria: } \\
\omega_{\text {tol }}[\mathrm{deg} / \mathrm{s}], \angle_{\text {tol }}[\mathrm{deg}]\end{array}$ & $\begin{array}{c}\text { Time to } \\
\text { Converge [min] }\end{array}$ & $\begin{array}{c}\text { Rate: }\|\omega\| \\
{[\mathrm{deg} / \mathrm{s}]}\end{array}$ & $\begin{array}{c}\text { Pointing } \\
\text { Error [deg] }\end{array}$ \\
\hline $5 \times 10^{-4}$ & $5 \times 10^{-6}$ & 2000 & $2 \times 10^{-7}$ & $1 \times 10^{-3}$ & 518.165 & $5.45 \times 10^{-4}$ & 1.792 \\
\hline
\end{tabular}




\subsubsection{Wheel Spin Up Performance with Torque Control Noise}
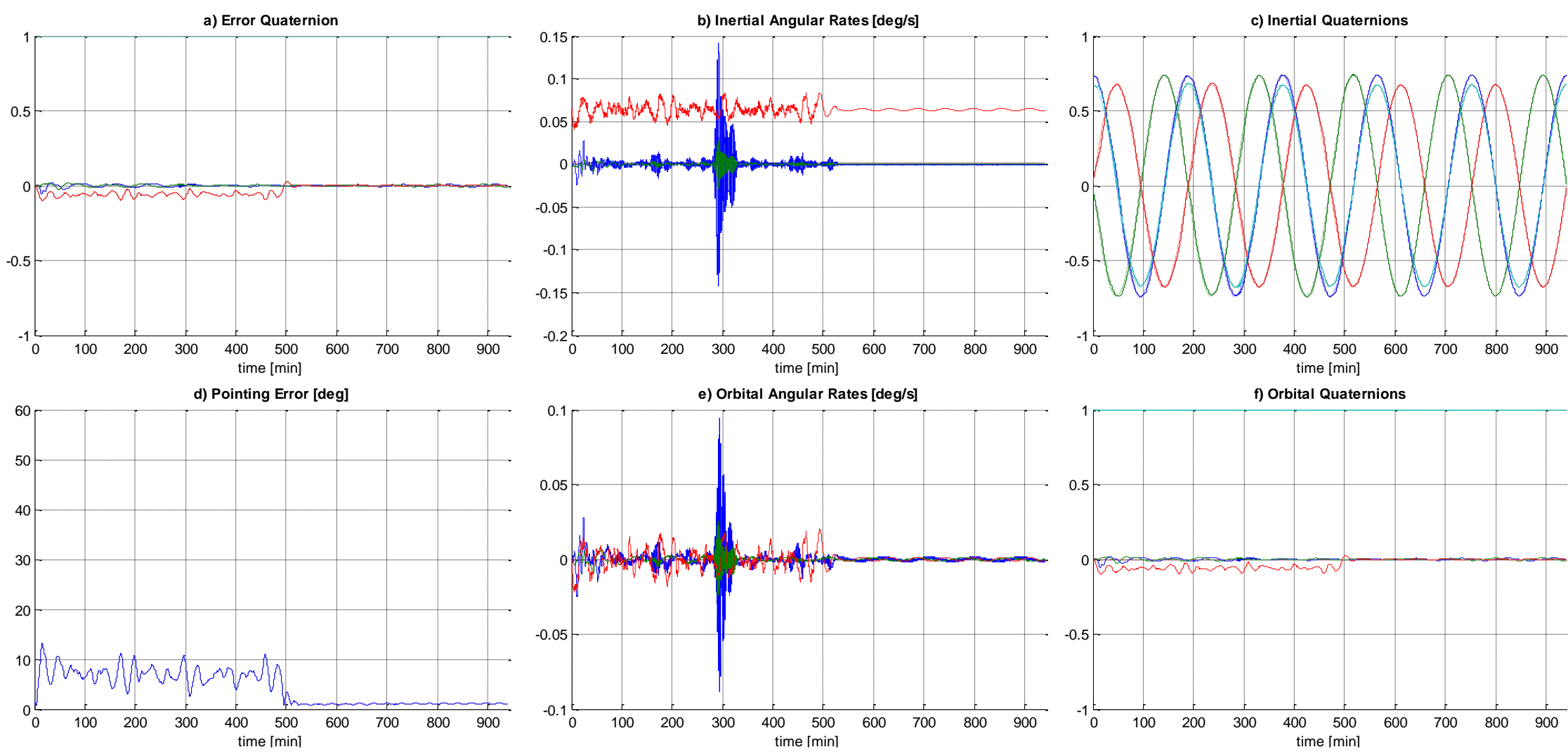

Figure 8.23: PD controller performance during wheel spin up with noise in torque control $\left(\sigma=1 \times 10^{-6}\right)$

\begin{tabular}{|c|c|c|c|c|c|c|c|}
\hline$D_{P D}$ & $K_{P D}$ & $\begin{array}{l}\text { Final Wheel } \\
\text { Speed [RPM] }\end{array}$ & $\begin{array}{c}\text { Spin Up } \\
\text { Torque }[\mathrm{Nm}]\end{array}$ & $\begin{array}{l}\text { Convergence Criteria: } \\
\omega_{\text {tol }}[\mathrm{deg} / \mathrm{s}], \angle_{\text {tol }}[\mathrm{deg}]\end{array}$ & $\begin{array}{c}\text { Time to } \\
\text { Converge }[\mathrm{min}]\end{array}$ & $\begin{array}{c}\text { Rate: }\|\omega\| \\
{[\mathrm{deg} / \mathrm{s}]}\end{array}$ & $\begin{array}{c}\text { Pointing } \\
\text { Error [deg] }\end{array}$ \\
\hline $5 \times 10^{-4}$ & $5 \times 10^{-6}$ & 2000 & $2 \times 10^{-7}$ & $1 \times 10^{-3}$ & 531.8317 & $5.37 \times 10^{-4}$ & 1.3122 \\
\hline
\end{tabular}




\subsection{Attitude Maintenance}

In the event that the spacecraft drifts from its target orientation during wheel spin up or the spacecraft loses pointing, the attitude control system must reacquire the target orientation. This is not a trivial task as the momentum wheel adds gyroscopic stiffness in the already coupled roll and yaw dynamics. With the added challenge of the non-linearity of magnetic actuation, reacquisition is not feasible with the momentum wheel running. The required procedure for reacquisition is thus as follows:

- The momentum wheel is despun slowly at a constant torque while opposed by the magnetorquers via the active magnetic control routine

- Once the momentum wheel has been brought to rest the active magnetic control routine will reacquire the target orientation

- Upon successful reacquisition of the target orientation the momentum wheel will be commanded to spin up back to the desired speed at a constant torque while opposed by the magnetorquers

This procedure was simulated with initial angular rates the same as the initial wheel spin up as

$$
\omega_{0}=\left[\begin{array}{lll}
-1.9685 \times 10^{-6} & -1.7453 \times 10^{-5} & 0.0011
\end{array}\right]
$$

The initial orientation however was set to represent an 11 degree offset in the yaw and pitch axes

$$
q_{0}=\left[\begin{array}{llll}
0.7243 & -0.0059 & 0.1348 & 0.6762
\end{array}\right]
$$

The simulation was run for 15 orbits with the wheel initially set to spin down with the PD controller active and the convergence check off until the wheel was off. The controller was then allowed to reacquire the ram pointing orientation per the convergence criteria 
established previously and the wheel set to spin up following the same procedure as the initial spin up. As can be seen in the following section, the spacecraft loses pointing as the wheel is spun down but promptly reacquires the target orientation and performs in a similar fashion to the previous section. The noise from the wheel torque controller was included for a more realistic simulation. 


\subsubsection{Reacquisition}
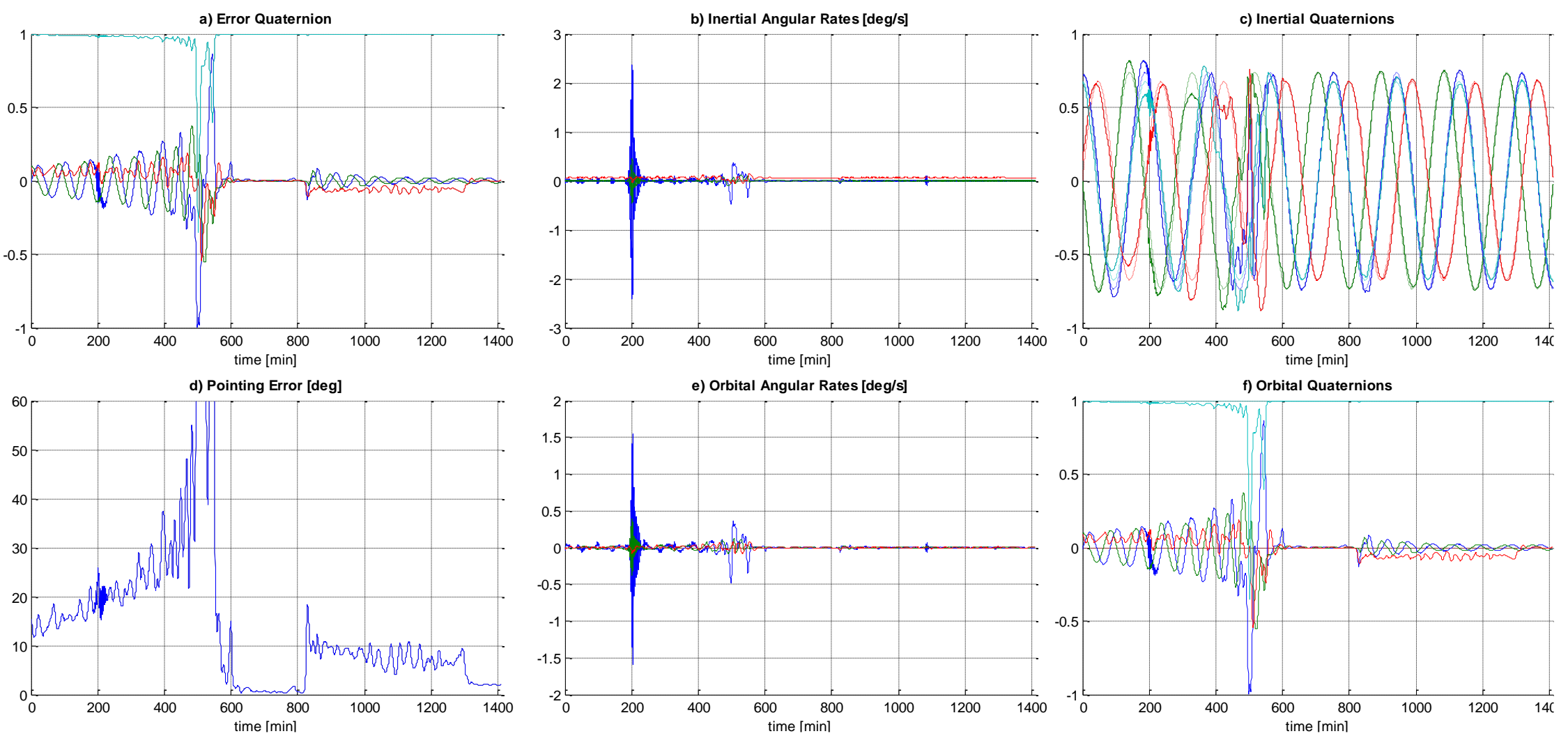

Figure 8.24: PD controller performance for reacquisition of pointing with torque control noise $\left(\sigma=1 \times 10^{-6}\right)$

\begin{tabular}{|c|c|c|c|c|c|c|c|}
\hline$D_{P D}$ & $K_{P D}$ & $\begin{array}{l}\text { Final Wheel } \\
\text { Speed [RPM] }\end{array}$ & $\begin{array}{c}\text { Spin Up } \\
\text { Torque [Nm] }\end{array}$ & $\begin{array}{l}\text { Convergence Criteria: } \\
\omega_{t o l}[\mathrm{deg} / \mathrm{s}], \angle_{t o l}[\mathrm{deg}]\end{array}$ & $\begin{array}{c}\text { Time to } \\
\text { Converge [min] }\end{array}$ & $\begin{array}{c}\text { Rate: }\|\omega\| \\
{[\mathrm{deg} / \mathrm{s}]}\end{array}$ & $\begin{array}{c}\text { Pointing } \\
\text { Error [deg] }\end{array}$ \\
\hline $5 \times 10^{-4}$ & $5 \times 10^{-6}$ & 2000 & $2 \times 10^{-7}$ & $1 \times 10^{-3}$ & 1341.8 & $9.00 \times 10^{-4}$ & 1.9978 \\
\hline
\end{tabular}




\section{Conclusions}

A combination of a gravity gradient system with a momentum bias wheel was proposed to meet pointing requirements while reducing power requirements and overall system complexity. A MATLAB simulation of dynamic and kinematic behavior of the system in orbit was implemented to guide system design and verify that the pointing requirements will be met. The problem was broken into four phases: detumbling, initial attitude acquisition, wheel spin-up, and attitude maintenance.

For the initial phase of detumbling, the B-dot controller has been shown to be a simple but thoroughly robust method. The results show that it is stable for a wide range of gains and pulse times. The controller is directly limited by the approximation of the body angular rates using the change in the magnetic field. This limitation is most evident when the pulse time is too long, magnetometer resolution too low, or magnetometer noise too high. The final angular rates achieved by the controller are comparable to those of previous $1 \mathrm{U}$ designs and is thus a scalable solution for all future CubeSat missions (Guerrant). Table 9.1 summarizes the results of the simulations and optimum settings for the system simulated.

Table 9.1: Summary of detumbling performance

\begin{tabular}{|c|c|c|c|c|c|c|c|}
\hline & \multirow{2}{*}{$K_{B d o t}$} & \multirow{2}{*}{$\begin{array}{l}\text { Pulse } \\
\text { Time } \\
{[\mathrm{s}]}\end{array}$} & \multirow{2}{*}{$\begin{array}{l}\text { Convergence } \\
\text { Criteria [T/s] }\end{array}$} & \multicolumn{2}{|c|}{ Magnetometer } & \multirow[b]{2}{*}{$\begin{array}{c}\text { Final } \\
\text { Body- } \\
\text { Inertial } \\
\text { Rates } \\
{[\mathrm{deg} / \mathrm{s}]}\end{array}$} & \multirow{2}{*}{$\begin{array}{r}\text { Total } \\
\text { Charge } \\
\text { Draw } \\
\text { [Ah] }\end{array}$} \\
\hline & & & & $\begin{array}{c}\text { Resolution } \\
{[\mathrm{T}]}\end{array}$ & $\begin{array}{c}\text { Noise } \\
(\text { Standard } \\
\text { Deviation } \sigma \text { ) } \\
{[\mathrm{T}]}\end{array}$ & & \\
\hline $\begin{array}{l}\text { Stable } \\
\text { Range }\end{array}$ & $\begin{array}{l}2 \times 10^{4} \text { to } \\
3 \times 10^{5}\end{array}$ & $\begin{array}{l}0.9 \text { to } \\
11.9 \\
\end{array}$ & $\begin{array}{l}3 \times 10^{-7} \text { to } \\
7 \times 10^{-7}\end{array}$ & $<5 \times 10^{-5}$ & $<3 \times 10^{-5}$ & $\begin{array}{l}0.1823 \\
\text { (mean) }\end{array}$ & $\begin{array}{l}4.2339 \\
(\text { mean) }\end{array}$ \\
\hline Optimum & $6 \times 10^{4}$ & 9.9 & $4 \times 10^{-7}$ & $\leq 1 \times 10^{-5}$ & $<3 \times 10^{-6}$ & 0.1742 & 3.0892 \\
\hline
\end{tabular}


Achieving low body-inertial rates through the use of B-dot effectively reduces the requirements on the controller for initial acquisition while incorporating a flight heritage controller.

For the initial acquisition phase, the PD controller performed well and successfully aligned the satellite with its target ram-pointing orientation in the orbital frame. It was discovered that the non-linear nature of magnetic actuation precludes the use of the suggested linear design techniques for global stability. Instead it was found that a line search technique was required to find an optimal gain combination. The line search technique was a feasible method of gain selection in part due to the relative simplicity of the controller design and the direct correlation between rate and orientation and their respective gains. The following table summarizes the selections and performance of the optimal PD controller for initial acquisition.

Table 9.2: Summary of ideal case initial acquisition performance

\begin{tabular}{|c|c|c|c|c|c|c|c|}
\hline \multirow[t]{2}{*}{ Rate Gain } & \multirow{2}{*}{$\begin{array}{l}\text { Orientation } \\
\text { Gain }\end{array}$} & \multirow{2}{*}{$\begin{array}{l}\text { Pulse } \\
\text { Time } \\
{[\mathrm{s}]}\end{array}$} & \multicolumn{2}{|c|}{ Convergence Criteria } & \multirow{2}{*}{$\begin{array}{c}\text { Final Body- } \\
\text { Inertial Rate } \\
{[\mathrm{deg} / \mathrm{s}]}\end{array}$} & \multirow{2}{*}{$\begin{array}{c}\text { Pointing } \\
\text { Error } \\
{[\mathrm{deg}]}\end{array}$} & \multirow{2}{*}{$\begin{array}{c}\text { Time to } \\
\text { Converge } \\
\text { [min] }\end{array}$} \\
\hline & & & $\begin{array}{c}\text { Rate } \\
{[\mathrm{deg} / \mathrm{s}]}\end{array}$ & $\begin{array}{l}\text { Pointing } \\
\text { [deg] }\end{array}$ & & & \\
\hline $8 \times 10^{-4}$ & $5 \times 10^{-6}$ & 9.9 & 0.001 & 2 & $5.74 \times 10^{-4}$ & 1.1883 & 197.165 \\
\hline
\end{tabular}

In order to find the requirements of the attitude determination algorithm, errors due to resolution and noise were independently simulated for the rate and orientation solutions. The following table summarizes the minimum resolution and maximum noise for which the controller remains stable and the recommended values for avoiding an impact on performance. 
Table 9.3: Summary of Attitude Determination Algorithm Requirements

\begin{tabular}{|l|c|c|c|c|}
\hline \multirow{2}{*}{} & \multicolumn{2}{|c|}{ Rate Determination } & \multicolumn{2}{c|}{ Orientation Determination } \\
\cline { 2 - 5 } & Resolution [rad/s] & $\begin{array}{c}\text { Noise (Standard } \\
\text { Deviation } \sigma) \\
{[\mathrm{rad} / \mathrm{s}]}\end{array}$ & $\begin{array}{c}\text { Resolution } \\
\text { Deviation } \sigma \text { ) }\end{array}$ \\
\hline Stable & $<1 \times 10^{-4}$ & $<1 \times 10^{-4}$ & $<1 \times 10^{-1}$ & $<5 \times 10^{-2}$ \\
\hline Recommended & $<1 \times 10^{-7}$ & $<1 \times 10^{-5}$ & $<1 \times 10^{-5}$ & $<1 \times 10^{-5}$ \\
\hline
\end{tabular}

The sensitivity of the controller to the rate resolution and noise far outweighs the impact of orientation errors. This suggests that if the attitude determination algorithm cannot calculate sufficiently accurate rates from the position sensor suite, a high accuracy threeaxis gyroscope should be included.

For the wheel spin up phase, the PD controller needed to be modified to exclude the gyroscopic torque. The rate gain also needed to be reduced to avoid the controller from overreacting to the fluctuations in angular rate. After modification, the controller was able to maintain pointing while the momentum wheel was spun up at a constant torque until the desired speed was reached. Even with the inclusion of noise in the torque control for the momentum wheel, the PD controller was able to maintain pointing. The following table summarizes the settings and performance of the PD controller during wheel spin up

Table 9.4: Summary of wheel spin up performance

\begin{tabular}{|c|c|c|l|l|l|l|l|}
\hline Rate Gain & $\begin{array}{l}\text { Orientation } \\
\text { Gain }\end{array}$ & $\begin{array}{l}\text { Final } \\
\text { Wheel } \\
\text { Speed } \\
{[\mathrm{RPM}]}\end{array}$ & $\begin{array}{l}\text { Spin Up } \\
\text { Torque } \\
{[\mathrm{Nm}]}\end{array}$ & $\begin{array}{l}\text { Wheel } \\
\text { Torque } \\
\text { Noise } \sigma \\
{[\mathrm{Nm}]}\end{array}$ & $\begin{array}{l}\text { Final Body- } \\
\text { Inertial Rate } \\
{[\mathrm{deg} / \mathrm{s}]}\end{array}$ & $\begin{array}{l}\text { Pointing } \\
\text { Error }[\mathrm{deg}]\end{array}$ & $\begin{array}{l}\text { Time to } \\
\text { Converge } \\
{[\mathrm{min}]}\end{array}$ \\
\hline $5 \times 10^{-4}$ & $5 \times 10^{-6}$ & 2000 & $2 \times 10^{-7}$ & $1 \times 10^{-6}$ & $5.37 \times 10^{-4}$ & 1.3122 & 531.8317 \\
\hline
\end{tabular}


Once the momentum wheel has spun up, the satellite is in a thoroughly stable configuration with the gyroscopic stiffness of the pitch wheel resisting yaw and roll disturbances and the gravity gradient torques resisting pitch and roll disturbances.

For the final phase of the problem, reacquisition, it was found that the controller could not overcome the stability of the system once the wheel was spun up. Thus it was determined that in the event of a loss of pointing, the wheel must be despun using the same settings as wheel spin up, the target orientation reacquired as it was in the initial acquisition, and the wheel spun up again. The successful simulation of this reacquisition of the target orientation speaks to the robustness of the control architecture.

Ultimately, this configuration for attitude control in a CubeSat could be applied to many future missions with the simulation serving as a design tool for CubeSat developers. By choosing a passively stable architecture with active elements, the attitude control can be tailored to the requirements of the mission. The incorporation of detumbling using the flight heritage B-dot algorithm with a simple PD controller lowers the overall complexity of Cal Poly's first three-axis controlled CubeSat. Successful demonstration of this control architecture will pave the way for added performance and capability for future missions. 


\section{Future Work}

\subsection{Hardware in the loop}

In order to harness the full potential of the simulation as an effective design and validation tool, the simulation developed in this thesis could be further modified to operate hardware in the loop. The code would need to be modified to incorporate flight hardware running flight software through the use of MATLAB's MEX functions. A computer running the simulation in MATLAB would model the space environment and vehicle dynamics to generate sensor inputs and pass them via serial connection to the avionics hardware. The avionics would be running its flight software and the attitude determination and control routines to interpret and respond to the simulated sensor information. The flight software in turn would output control commands back to the computer running the simulation via the serial connection to simulated magnetorquers and a momentum wheel. The end result would be a system level test of the ADCS flight software that could prove a powerful development tool that would allow developers to catch software or controller design flaws before delivery.

\subsection{Attitude Determination Algorithm}

The current simulation broadly accounts for errors in the attitude determination algorithm by modeling it as noise added to the actual orientation and body rates. The future work in developing the attitude determination algorithm for ExoCube could be incorporated into the simulation for a more realistic assessment of error. Incorporating an Extended 
Kalman Filter (EKF) would make the simulation presented in this thesis a more robust design tool.

\subsection{Pure Momentum Bias}

Although the gravity gradient, momentum bias control architecture provides a robust solution for ExoCube's pointing requirements, the inclusion of deployables significantly complicated the mechanical design. With the addition of a pitch control algorithm, the deployable booms could be eliminated thus reducing the mechanical complexity at the cost of increased software complexity. As ExoCube is PolySat's first attempt at a three axis controlled CubeSat, the mechanical complexity of deployable booms was deemed acceptable with the notion that future three axis controlled CubeSats could build on the lessons learned from ExoCube and possibly use a pure momentum bias control architecture. 


\section{References}

Bender, Erich. An Analysis of Stabilizing 3U CubeSats Using Gravity Gradient

Techniques and a Low Power Reaction Wheel. Senior Project. San Luis Obispo:

California Polytechnic State University, 2011. PDF.

Bowen, John Arthur. On-Board Orbit Determination and 3-Axis Attitude Determination for Picosatellite Applications. Masters Thesis. San Luis Obispo: California Polytechnic State University, 2009. PDF.

Brucat, PJ. Kinetic Molecular Theory. n.d. Web site. 19 February 2013. <http://www.chem.ufl.edu/ itl/2045/lectures/lec_d.html>.

Curtis, Howard D. Orbital Mechanics for Engineering Students. 2nd. Burlington: Elsevier Ltd., 2010. Textbook.

Eagle, C. David. Shadow Consitions of Earth Satellites. 2013. PDF. 15 March 2013. <http://www.cdeagle.com/html/ommatlab.html>.

Groÿekatthöfer, Karsten and Zizung Yoon. Introduction into quaternions for spacecraft attitude representation. Technical Paper. Technical University of Berlin. Berlin, 2012. PDF.

Guelman, M., et al. "Design and testing of magnetic controllers for Satellite stabilization." Acta Astronautica (2005): 231-239. PDF. 
Guerrant, Daniel Vernon. Design and Analysis of Fully Magnetic Control for Picosatellite Stabilization. Masters Thesis. San Luis Obispo: California Polytechnic State University, 2005. PDF.

Hall, Dr. Christopher. Spacecraft Attitude Dynamics and Control. 2003. PDF.

Munakata, Riki. "CubeSat Design Specification Rev. 12." 1 August 2009. CubeSat. Document. 26 March 2013. <cubesat.org>.

Philip, Adam. Attitude Sensing, Actuation, and Control of the BRITE and CanX-4\&5 Satellites. Masters Thesis. Toronto: University of Toronto, 2008. PDF.

Picone, J.M., et al. NRLMSISE-00: A New Empirical Model of the Atmosphere. 2003. Website. 5 December 2012. <http://www.nrl.navy.mil/research/nrlreview/2003/atmospheric-science/picone/>.

Schaub, Hanspeter and John L. Junkins. Analytical Mechanics of Aerospace Systems. 2nd. Reston: AIAA, 2002. Textbook.

Sidi, Marcel J. Spacecraft Dynamics and Control: A Practical Engineering Approach. New York: Cambridge University Press, 1997. Textbook.

Sinclair, Doug. Personal Correspondance Re: Sinclair Wheel Torque Control Mode Ryan Sellers. 21 March 2013. Email.

Smith, John. "f107_aph.m." F10.7 Solar Flux \& Ap Indices. MATLAB Central File Exchange, 13 February 2012. m file. 
$<$ http://www.mathworks.com/matlabcentral/fileexchange/35054-f10-7-solar-fluxap-indices>.

Stern, Dr. David P. The Moon: the Distant View. 17 September 2004. Website. 20 June 2012. 〈http://www-spof.gsfc.nasa.gov/stargaze/Smoon.htm>.

Sturm II, Erick Jonathan. Magnetic Attitude Estimation of a Tumbling Spacecraft. Masters Thesis. San Luis Obispo: California Polytechnic State University, 2005. PDF.

Technical Committee ISO/TC 20, Aircraft and space vehicles, Subcommittee 14, Space systems and operations. Earth's Internal Magnetic Reference Field Models. International Standard ISO Guide. Geneva: ISO, 2009. PDF.

Turner, Andrew J. An Open-Source, Extensible Spacecraft Simulation and Modeling Environment Framework. Masters Thesis. Blacksburg: Virgina Polytechnic Institute and State University, 2003. PDF.

United States Naval Observatory. The Astronomical Almanac. 2013. Web Page. 5 January 2013. <http://asa.usno.navy.mil/>.

Varma, Surjit. Control of Satellites Using Environmental Forces: Aerodynamic Drag/Solar Radiation Pressure. PhD Dissertation. Toronto: Ryerson University, 2011. PDF.

Wertz, James R. Spacecraft Attitude Determination and Control. Norwell: Kluwer Academic Publishers, 1978. Textbook. 
Wertz, James R., David F. Everett and Jeffery J. Puschell. Space Mission Engineering: The New SMAD. Hawthorne: Microcosm Press, 2011. Textbook.

Wie, B., H. Weiss and A. Arapostathis. A Quaternion Feedback Regulator for Spacecraft Eigenaxis Rotations. Technical Paper. Reston: AIAA, n.d. PDF.

Wie, Bong. Space Vehicle Dynamics and Control. Reston: AIAA, 1998. Textbook. 
Appendices
A. B-dot Controller Simulation Data
B. PD Controller Simulation Data

Page 121 


\section{APPENDIX A: B-dot Controller Gain Selection}

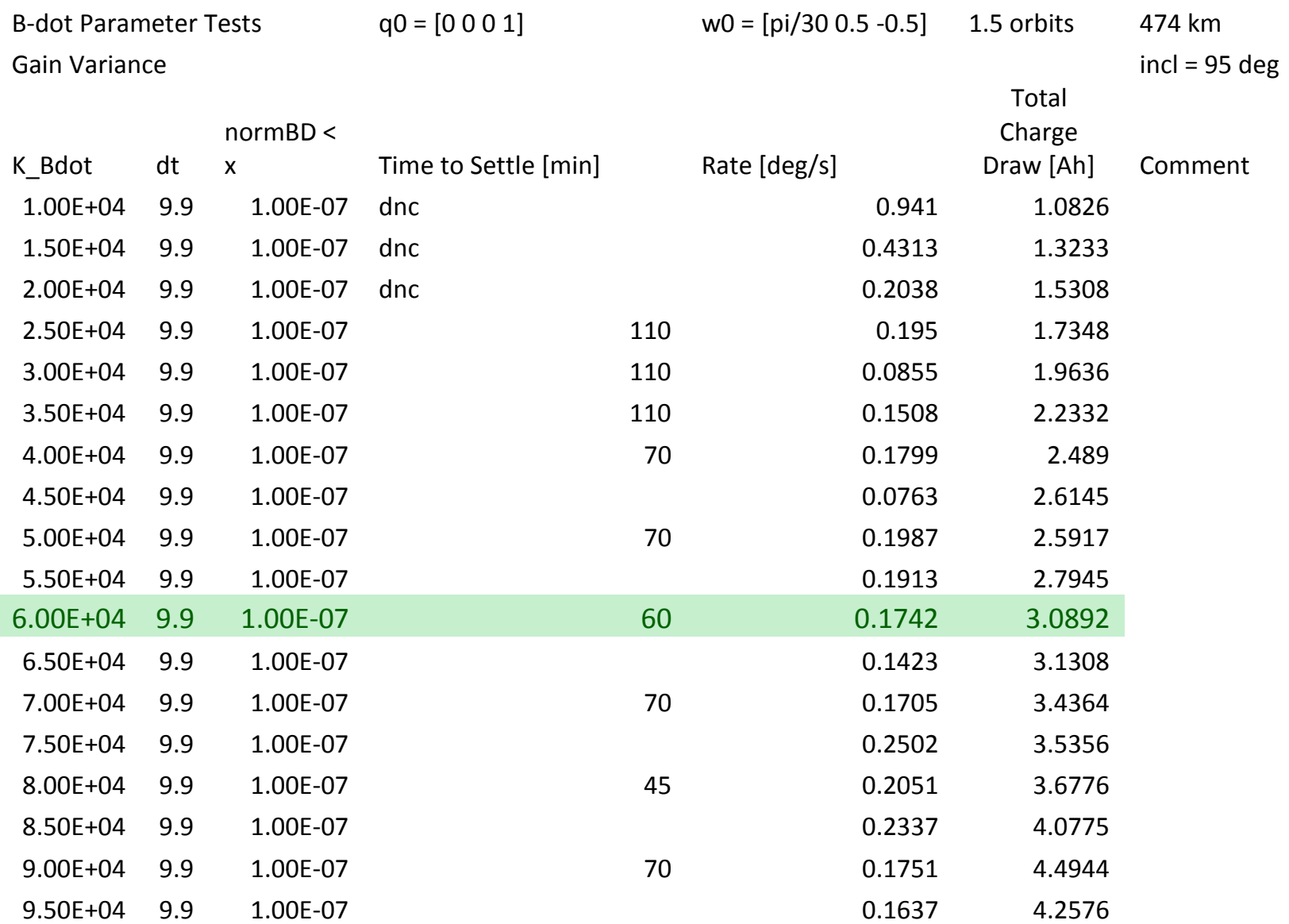




$\begin{array}{lll}1.00 \mathrm{E}+05 & 9.9 & 1.00 \mathrm{E}-07 \\ 1.50 \mathrm{E}+05 & 9.9 & 1.00 \mathrm{E}-07 \\ 2.00 \mathrm{E}+05 & 9.9 & 1.00 \mathrm{E}-07 \\ 2.50 \mathrm{E}+05 & 9.9 & 1.00 \mathrm{E}-07 \\ 3.00 \mathrm{E}+05 & 9.9 & 1.00 \mathrm{E}-07 \\ 3.50 \mathrm{E}+05 & 9.9 & 1.00 \mathrm{E}-07 \\ 4.00 \mathrm{E}+05 & 9.9 & 1.00 \mathrm{E}-07 \\ 5.00 \mathrm{E}+05 & 9.9 & 1.00 \mathrm{E}-07 \\ 6.00 \mathrm{E}+05 & 9.9 & 1.00 \mathrm{E}-07 \\ 1.00 \mathrm{E}+06 & 9.9 & 1.00 \mathrm{E}-07 \\ 5.00 \mathrm{E}+06 & 9.9 & 1.00 \mathrm{E}-07 \\ 1.00 \mathrm{E}+07 & 9.9 & 1.00 \mathrm{E}-07\end{array}$

30

30

40

$\begin{array}{rrl}0.1608 & 4.6094 & \\ 0.2096 & 7.4571 & \\ 0.2433 & 8.3793 & \\ 0.1728 & 9.787 & \\ 0.2668 & 11.0283 & \\ & & \text { Local } \\ 0.1582 & 14.4061 & \begin{array}{l}\text { Instability } \\ \text { Local }\end{array} \\ 0.2327 & 13.078 & \begin{array}{l}\text { Instability } \\ \text { Local }\end{array} \\ 0.2792 & 1.83 E+01 & \begin{array}{l}\text { Instability } \\ \text { Local }\end{array} \\ 0.2887 & 1.84 \mathrm{E}+01 & \begin{array}{l}\text { Instability } \\ \text { Local }\end{array} \\ 0.187 & 2.08 \mathrm{E}+01 & \begin{array}{l}\text { Instability } \\ \text { Local }\end{array} \\ 0.0994 & 2.51 \mathrm{E}+01 & \begin{array}{l}\text { Instability } \\ \text { Local }\end{array} \\ 0.2342 & 2.22 \mathrm{E}+01 & \text { Instability }\end{array}$


B-dot Controller Pulse Time Selection

\begin{tabular}{|c|c|c|c|c|c|c|}
\hline \multicolumn{7}{|c|}{ B-dot Parameter } \\
\hline Pulse Variance & & & & & & incl $=95 \mathrm{deg}$ \\
\hline K Bdot & $d t$ & $\begin{array}{l}\text { normBD }< \\
x\end{array}$ & Time to Settle [min] & Rate $[\mathrm{deg} / \mathrm{s}$ ] & $\begin{array}{c}\text { Total } \\
\text { Charge } \\
\text { Draw [Ah] }\end{array}$ & Comment \\
\hline $6.00 E+04$ & 0.9 & 1.00E-07 & 80 & 0.1496 & 6.2237 & \\
\hline $6.00 E+04$ & 1.9 & $1.00 \mathrm{E}-07$ & 60 & 0.2096 & 5.5699 & \\
\hline $6.00 E+04$ & 2.9 & $1.00 \mathrm{E}-07$ & 60 & 0.2126 & 5.4038 & \\
\hline $6.00 E+04$ & 3.9 & $1.00 \mathrm{E}-07$ & 80 & 0.135 & 4.5776 & \\
\hline $6.00 E+04$ & 4.9 & $1.00 \mathrm{E}-07$ & 80 & 0.1782 & 4.8328 & \\
\hline $6.00 E+04$ & 5.9 & $1.00 \mathrm{E}-07$ & 60 & 0.2365 & 4.4816 & \\
\hline $6.00 E+04$ & 6.9 & $1.00 \mathrm{E}-07$ & 80 & 0.1153 & 4.1337 & \\
\hline $6.00 E+04$ & 7.9 & $1.00 \mathrm{E}-07$ & 60 & 0.1333 & 3.6123 & \\
\hline $6.00 \mathrm{E}+04$ & 8.9 & $1.00 \mathrm{E}-07$ & 80 & 0.2423 & 3.2976 & \\
\hline $6.00 E+04$ & 9.9 & 1.00E-07 & 60 & 0.1742 & 3.0892 & \\
\hline $6.00 E+04$ & 10.9 & $1.00 \mathrm{E}-07$ & 80 & 0.2581 & 2.8712 & \\
\hline $6.00 E+04$ & 11.9 & $1.00 \mathrm{E}-07$ & 70 & 0.2058 & 2.6828 & \\
\hline $6.00 E+04$ & 12.4 & $1.00 \mathrm{E}-07$ & 80 & 0.2102 & 2.809 & \\
\hline $6.00 E+04$ & 12.5 & $1.00 \mathrm{E}-07$ & dnc & 21.3533 & 10.0032 & diverges \\
\hline $6.00 E+04$ & 12.6 & $1.00 \mathrm{E}-07$ & dnc & 21.22 & 10.0724 & diverges \\
\hline $6.00 \mathrm{E}+04$ & 12.9 & $1.00 \mathrm{E}-07$ & dnc & 20.6812 & 9.8866 & diverges \\
\hline
\end{tabular}

Page 124 
B-dot Controller Convergence Criteria Selection

\begin{tabular}{|c|c|c|c|c|c|c|}
\hline \multicolumn{3}{|l|}{$\begin{array}{l}\text { B-dot Parameter } \\
\text { Tests }\end{array}$} & $q 0=\left[\begin{array}{llll}0 & 0 & 0 & 1\end{array}\right]$ & w0 = [pi/30 0.5 - 0.5$]$ & 5 orbits & \multirow{2}{*}{$\begin{array}{l}475 \mathrm{~km} \\
\text { incl = } 95 \mathrm{deg}\end{array}$} \\
\hline Convergence Cri & teria & /ariance & & & & \\
\hline K_Bdot & $\mathrm{dt}$ & $\begin{array}{l}\text { normBD }< \\
x\end{array}$ & Time to Settle [min] & Rate [deg/s] & $\begin{array}{c}\text { Total } \\
\text { Charge } \\
\text { Draw [Ah] }\end{array}$ & Comment \\
\hline $6.00 \mathrm{E}+04$ & 9.9 & $1.00 \mathrm{E}-07$ & dnc & 0.1684 & 8.5026 & \\
\hline $6.00 E+04$ & 9.9 & $2.00 \mathrm{E}-07$ & dnc & 0.1684 & 8.5026 & \\
\hline $6.00 E+04$ & 9.9 & $2.50 \mathrm{E}-07$ & dnc & 0.1684 & 8.5026 & \\
\hline $6.00 E+04$ & 9.9 & $3.00 \mathrm{E}-07$ & 181.4983 & 0.1539 & 3.6621 & \\
\hline $6.00 \mathrm{E}+04$ & 9.9 & $3.50 \mathrm{E}-07$ & 145.165 & 0.1757 & 3.1246 & \\
\hline $6.00 \mathrm{E}+04$ & 9.9 & 4.00E-07 & 82.165 & 0.1301 & 2.13 & \\
\hline $6.00 \mathrm{E}+04$ & 9.9 & $4.50 \mathrm{E}-07$ & 49.4983 & 0.307 & 1.5551 & \\
\hline $6.00 E+04$ & 9.9 & $5.00 \mathrm{E}-07$ & 45.8317 & 0.3464 & 1.5076 & \\
\hline $6.00 E+04$ & 9.9 & 5.50E-07 & 43.4983 & 0.3783 & 1.4694 & \\
\hline $6.00 \mathrm{E}+04$ & 9.9 & $6.00 \mathrm{E}-07$ & 42.165 & 0.4202 & 1.4515 & \\
\hline $6.00 \mathrm{E}+04$ & 9.9 & $6.50 \mathrm{E}-07$ & 39.8317 & 0.5117 & 1.417 & \\
\hline $6.00 E+04$ & 9.9 & $7.00 \mathrm{E}-07$ & 39.8317 & 0.5117 & 1.417 & \\
\hline $6.00 E+04$ & 9.9 & $7.50 \mathrm{E}-07$ & 22.8317 & 0.9337 & 1.0726 & \\
\hline $6.00 E+04$ & 9.9 & $8.00 \mathrm{E}-07$ & 22.8317 & 0.9337 & 1.0726 & \\
\hline $6.00 \mathrm{E}+04$ & 9.9 & $8.50 \mathrm{E}-07$ & 22.8317 & 0.9337 & 1.0726 & \\
\hline $6.00 \mathrm{E}+04$ & 9.9 & $9.00 \mathrm{E}-07$ & 20.4983 & 0.985 & 1.035 & \\
\hline $6.00 E+04$ & 9.9 & $9.50 \mathrm{E}-07$ & 20.4983 & 0.985 & 1.035 & \\
\hline $6.00 \mathrm{E}+04$ & 9.9 & $1.00 \mathrm{E}-06$ & 20.4983 & 0.985 & 1.035 & \\
\hline
\end{tabular}

Page 125 
B-dot Controller Minimum Magnetometer Resolution

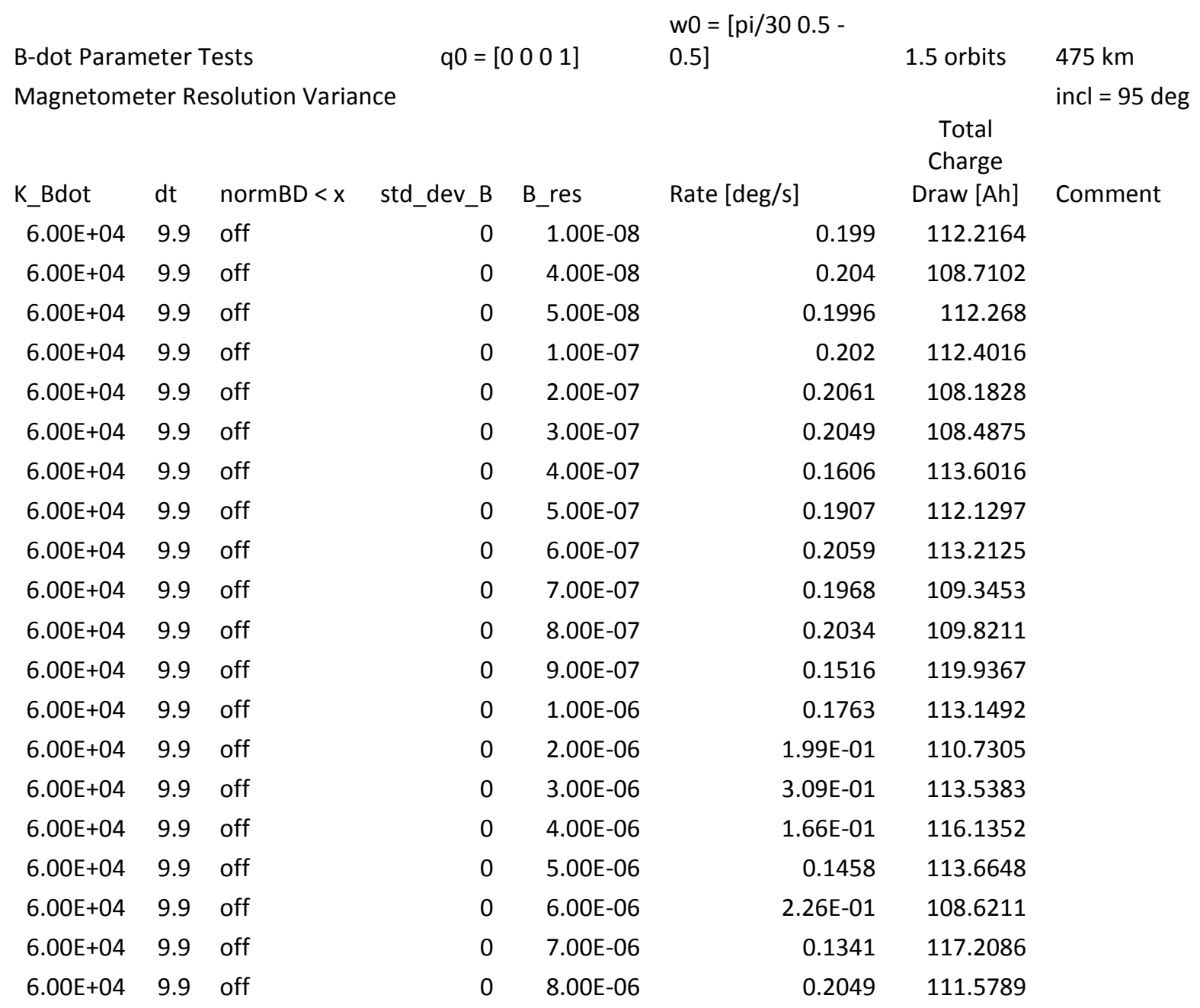

Page 126 


$\begin{array}{rllrrrrl}6.00 \mathrm{E}+04 & 9.9 & \text { off } & 0 & 9.00 \mathrm{E}-06 & 0.3559 & 109.9102 & \\ 6.00 \mathrm{E}+04 & 9.9 & \text { off } & 0 & 1.00 \mathrm{E}-05 & 0.6275 & 120.3094 & \text { visible b_body error } \\ 6.00 \mathrm{E}+04 & 9.9 & \text { off } & 0 & 1.25 \mathrm{E}-05 & 0.1101 & 116.1633 & \\ 6.00 \mathrm{E}+04 & 9.9 & \text { off } & 0 & 1.50 \mathrm{E}-05 & 0.1917 & 116.2148 & \\ 6.00 \mathrm{E}+04 & 9.9 & \text { off } & 0 & 1.75 \mathrm{E}-05 & 0.2819 & 134.6039 & \\ & & & & & & & \text { step behavior in yaw } \\ 6.00 \mathrm{E}+04 & 9.9 & \text { off } & 0 & 2.00 \mathrm{E}-05 & 0.17 & 133.8914 & \text { rates } \\ 6.00 \mathrm{E}+04 & 9.9 & \text { off } & 0 & 2.25 \mathrm{E}-05 & 0.3362 & 116.2336 & \\ 6.00 \mathrm{E}+04 & 9.9 & \text { off } & 0 & 2.50 \mathrm{E}-05 & 0.3983 & 132.8484 & \\ 6.00 \mathrm{E}+04 & 9.9 & \text { off } & 0 & 3.00 \mathrm{E}-05 & 0.2737 & 117.7688 & \\ 6.00 \mathrm{E}+04 & 9.9 & \text { off } & 0 & 3.25 \mathrm{E}-05 & 0.8892 & 122.6109 & \\ 6.00 \mathrm{E}+04 & 9.9 & \text { off } & 0 & 3.50 \mathrm{E}-05 & 0.8076 & 122.3531 & \\ 6.00 \mathrm{E}+04 & 9.9 & \text { off } & 0 & 3.75 \mathrm{E}-05 & 0.3192 & 130.7836 & \\ 6.00 \mathrm{E}+04 & 9.9 & \text { off } & 0 & 4.00 \mathrm{E}-05 & 0.1544 & 145.5656 & \\ 6.00 \mathrm{E}+04 & 9.9 & \text { off } & 0 & 4.25 \mathrm{E}-05 & 0.1862 & 126.1594 & \\ 6.00 \mathrm{E}+04 & 9.9 & \text { off } & 0 & 4.50 \mathrm{E}-05 & 0.9482 & 132.2578 & \\ 6.00 \mathrm{E}+04 & 9.9 & \text { off } & 1 & 4.75 \mathrm{E}-05 & 0.3845 & 103.425 & \\ 6.00 \mathrm{E}+04 & 9.9 & \text { off } & 0 & 5.00 \mathrm{E}-05 & 0.4814 & 112.1578 & \\ 6.00 \mathrm{E}+04 & 9.9 & \text { off } & & 27.2824 & 424.1391 & \text { complete divergence }\end{array}$

Page 127 
B-dot Controller Maximum Magnetometer Noise

\begin{tabular}{|c|c|c|c|c|c|c|c|}
\hline \multicolumn{3}{|l|}{$\begin{array}{l}\text { B-dot Parameter } \\
\text { Tests }\end{array}$} & \multicolumn{2}{|c|}{$\mathrm{q} 0=\left[\begin{array}{llll}0 & 0 & 0 & 1\end{array}\right]$} & w0 = [pi/30 0.5 -0.5] & 1.5 orbits & \multirow{2}{*}{$\begin{array}{l}475 \mathrm{~km} \\
\text { incl }=95 \mathrm{deg}\end{array}$} \\
\hline Magnetometer I & Noise & Variance & & & & & \\
\hline K_Bdot & $\mathrm{dt}$ & $\begin{array}{l}\text { normBD }< \\
x\end{array}$ & std_dev_B & B_res & Rate $[\mathrm{deg} / \mathrm{s}]$ & $\begin{array}{c}\text { Total } \\
\text { Charge } \\
\text { Draw [Ah] }\end{array}$ & Comment \\
\hline $6.00 \mathrm{E}+04$ & 9.9 & off & 0 & $1.00 \mathrm{E}-07$ & 0.202 & 112.4016 & \\
\hline $6.00 E+04$ & 9.9 & off & $1.00 \mathrm{E}-07$ & $1.00 \mathrm{E}-07$ & 0.1942 & 111.832 & \\
\hline $6.00 E+04$ & 9.9 & off & 2.00E-07 & $1.00 \mathrm{E}-07$ & 0.2012 & 112.9875 & \\
\hline $6.00 E+04$ & 9.9 & off & $3.00 \mathrm{E}-07$ & $1.00 \mathrm{E}-07$ & 0.2085 & 108.0844 & \\
\hline $6.00 \mathrm{E}+04$ & 9.9 & off & 4.00E-07 & $1.00 \mathrm{E}-07$ & 0.1906 & 111.3539 & \\
\hline $6.00 E+04$ & 9.9 & off & 5.00E-07 & $1.00 \mathrm{E}-07$ & 0.1795 & 110.2125 & \\
\hline $6.00 \mathrm{E}+04$ & 9.9 & off & $6.00 E-07$ & $1.00 \mathrm{E}-07$ & 0.2478 & 112.1109 & \\
\hline $6.00 E+04$ & 9.9 & off & 7.00E-07 & $1.00 \mathrm{E}-07$ & 0.146 & 115.6148 & \\
\hline $6.00 E+04$ & 9.9 & off & $8.00 \mathrm{E}-07$ & $1.00 \mathrm{E}-07$ & 0.2684 & 111.9563 & \\
\hline $6.00 \mathrm{E}+04$ & 9.9 & off & $9.00 E-07$ & $1.00 \mathrm{E}-07$ & 0.2334 & 115.0805 & \\
\hline $6.00 \mathrm{E}+04$ & 9.9 & off & $1.00 \mathrm{E}-06$ & $1.00 \mathrm{E}-07$ & 0.1979 & 112.3195 & \\
\hline $6.00 \mathrm{E}+04$ & 9.9 & off & 2.00E-06 & $1.00 \mathrm{E}-07$ & 0.2159 & 128.9531 & \\
\hline $6.00 \mathrm{E}+04$ & 9.9 & off & $3.00 \mathrm{E}-06$ & $1.00 \mathrm{E}-07$ & 0.2898 & 145.4508 & \\
\hline $6.00 E+04$ & 9.9 & off & $4.00 \mathrm{E}-06$ & $1.00 \mathrm{E}-07$ & 0.099 & 165.1945 & \\
\hline $6.00 E+04$ & 9.9 & off & 5.00E-06 & $1.00 \mathrm{E}-07$ & 0.4206 & 187.132 & \\
\hline $6.00 E+04$ & 9.9 & off & $6.00 \mathrm{E}-06$ & $1.00 \mathrm{E}-07$ & 0.1371 & 209.6156 & \\
\hline $6.00 E+04$ & 9.9 & off & $7.00 \mathrm{E}-06$ & $1.00 \mathrm{E}-07$ & 0.6753 & 229.95 & \\
\hline $6.00 \mathrm{E}+04$ & 9.9 & off & 8.00E-06 & $1.00 \mathrm{E}-07$ & 0.3291 & 249.5672 & \\
\hline $6.00 \mathrm{E}+04$ & 9.9 & off & $9.00 \mathrm{E}-06$ & $1.00 \mathrm{E}-07$ & 0.3857 & 270.1406 & \\
\hline $6.00 E+04$ & 9.9 & off & $1.00 \mathrm{E}-05$ & $1.00 \mathrm{E}-07$ & 0.5896 & 305.2711 & \\
\hline
\end{tabular}

Page 128 


$\begin{array}{lllllrrl}6.00 \mathrm{E}+04 & 9.9 & \text { off } & 1.50 \mathrm{E}-05 & 1.00 \mathrm{E}-07 & 1.0227 & 406.2445 & \\ 6.00 \mathrm{E}+04 & 9.9 & \text { off } & 2.00 \mathrm{E}-05 & 1.00 \mathrm{E}-07 & 1.1139 & 503.0883 & \\ 6.00 \mathrm{E}+04 & 9.9 & \text { off } & 2.50 \mathrm{E}-05 & 1.00 \mathrm{E}-07 & 1.4743 & 581.625 & \\ 6.00 \mathrm{E}+04 & 9.9 & \text { off } & 3.00 \mathrm{E}-05 & 1.00 \mathrm{E}-07 & 0.9003 & 635.9953 & \\ 6.00 \mathrm{E}+04 & 9.9 & \text { off } & 3.50 \mathrm{E}-05 & 1.00 \mathrm{E}-07 & 27.3258 & 726.7031 & \text { Complete Divergence } \\ 6.00 \mathrm{E}+04 & 9.9 & \text { off } & 4.00 \mathrm{E}-05 & 1.00 \mathrm{E}-07 & 1.9434 & 679.3078 & \text { Local instabilities } \\ 6.00 \mathrm{E}+04 & 9.9 & \text { off } & 4.50 \mathrm{E}-05 & 1.00 \mathrm{E}-07 & 25.5334 & 756.5883 & \text { Complete Divergence } \\ 6.00 \mathrm{E}+04 & 9.9 & \text { off } & 5.00 \mathrm{E}-05 & 1.00 \mathrm{E}-07 & 26.2234 & 736.2234 & \text { Complete Divergence } \\ 6.00 \mathrm{E}+04 & 9.9 & \text { off } & 1.00 \mathrm{E}-04 & 1.00 \mathrm{E}-07 & 2.76 \mathrm{E}+01 & 8.55 \mathrm{E}+02 & \text { Complete Divergence }\end{array}$

Page 129 


\section{APPENDIX B: PD Controller Line Search for Optimal Gains}

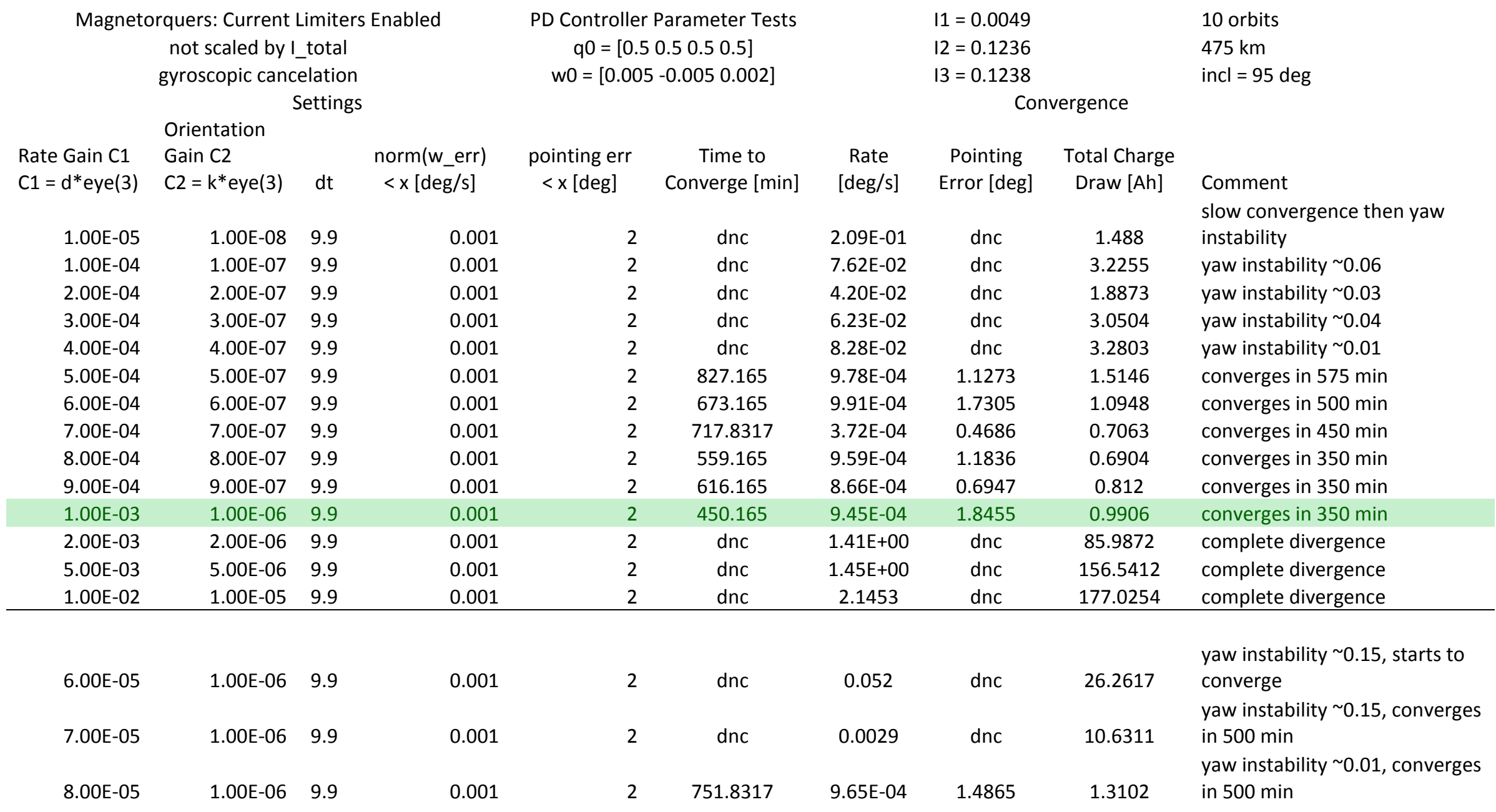

Page 130 


\begin{tabular}{|c|c|c|c|c|c|c|c|c|c|}
\hline $9.00 \mathrm{E}-05$ & $1.00 \mathrm{E}-06$ & 9.9 & 0.001 & 2 & dnc & 0.0098 & dnc & 1.3714 & $\begin{array}{l}\text { yaw instability } \sim 0.01 \text {, converges } \\
\text { in } 350 \text { min }\end{array}$ \\
\hline $1.00 \mathrm{E}-04$ & $1.00 \mathrm{E}-06$ & 9.9 & 0.001 & 2 & 716.165 & $9.82 \mathrm{E}-04$ & 1.7298 & 8.6956 & in $450 \mathrm{~min}$ \\
\hline $2.00 \mathrm{E}-04$ & $1.00 \mathrm{E}-06$ & 9.9 & 0.001 & 2 & 488.8317 & $9.85 \mathrm{E}-04$ & 0.9875 & 1.0926 & converges in $350 \mathrm{~min}$ \\
\hline $3.00 E-04$ & $1.00 \mathrm{E}-06$ & 9.9 & 0.001 & 2 & 570.165 & $9.73 \mathrm{E}-04$ & 1.7534 & 0.8962 & converges in $450 \mathrm{~min}$ \\
\hline 4.00E-04 & $1.00 \mathrm{E}-06$ & 9.9 & 0.001 & 2 & 622.165 & $8.74 \mathrm{E}-04$ & 1.5489 & 0.7821 & converges in $250 \mathrm{~min}$ \\
\hline $5.00 \mathrm{E}-04$ & $1.00 \mathrm{E}-06$ & 9.9 & 0.001 & 2 & 613.4983 & $7.58 \mathrm{E}-04$ & 0.8117 & 0.5025 & converges in $125 \mathrm{~min}$ \\
\hline $6.00 \mathrm{E}-04$ & $1.00 \mathrm{E}-06$ & 9.9 & 0.001 & 2 & 571.165 & $8.70 E-04$ & 1.8629 & 0.732 & converges in $450 \mathrm{~min}$ \\
\hline 7.00E-04 & $1.00 \mathrm{E}-06$ & 9.9 & 0.001 & 2 & 551.165 & $9.89 \mathrm{E}-04$ & 0.9282 & 0.7313 & converges in $150 \mathrm{~min}$ \\
\hline 8.00E-04 & $1.00 \mathrm{E}-06$ & 9.9 & 0.001 & 2 & 570.8317 & $9.79 E-04$ & 0.8323 & 0.7265 & converges in $250 \mathrm{~min}$ \\
\hline $9.00 \mathrm{E}-04$ & $1.00 \mathrm{E}-06$ & 9.9 & 0.001 & 2 & 439.165 & $8.76 \mathrm{E}-04$ & 1.0468 & 0.7636 & converges in $325 \mathrm{~min}$ \\
\hline $1.00 \mathrm{E}-03$ & $1.00 \mathrm{E}-06$ & 9.9 & 0.001 & 2 & 450.165 & $9.45 \mathrm{E}-04$ & 1.8455 & 0.9906 & converges in $350 \mathrm{~min}$ \\
\hline $2.00 \mathrm{E}-03$ & $1.00 \mathrm{E}-06$ & 9.9 & 0.001 & 2 & dnc & $1.52 \mathrm{E}-01$ & dnc & 83.7954 & diverges \\
\hline $3.00 \mathrm{E}-03$ & $1.00 \mathrm{E}-06$ & 9.9 & 0.001 & 2 & dnc & $3.46 \mathrm{E}-02$ & dnc & 135.5382 & diverges \\
\hline $9.00 \mathrm{E}-04$ & $9.00 \mathrm{E}-07$ & 9.9 & 0.001 & 2 & 616.165 & $8.66 \mathrm{E}-04$ & 0.6947 & 0.812 & converges in $350 \mathrm{~min}$ \\
\hline $9.00 \mathrm{E}-04$ & $1.00 \mathrm{E}-06$ & 9.9 & 0.001 & 2 & 439.165 & $8.76 \mathrm{E}-04$ & 1.0468 & 0.7636 & converges in $325 \mathrm{~min}$ \\
\hline $9.00 \mathrm{E}-04$ & $2.00 \mathrm{E}-06$ & 9.9 & 0.001 & 2 & 438.165 & $8.90 E-04$ & 0.7477 & 0.863 & converges in $200 \mathrm{~min}$ \\
\hline $9.00 \mathrm{E}-04$ & $3.00 \mathrm{E}-06$ & 9.9 & 0.001 & 2 & 341.165 & $5.80 \mathrm{E}-04$ & 0.5987 & 0.8867 & converges in $150 \mathrm{~min}$ \\
\hline $9.00 \mathrm{E}-04$ & 4.00E-06 & 9.9 & 0.001 & 2 & 302.165 & $9.68 \mathrm{E}-04$ & 0.8217 & 2.9356 & converges in $250 \mathrm{~min}$ \\
\hline $9.00 \mathrm{E}-04$ & $5.00 \mathrm{E}-06$ & 9.9 & 0.001 & 2 & 617.4983 & $4.80 \mathrm{E}-04$ & 1.0165 & 4.3888 & converges in $425 \mathrm{~min}$ \\
\hline $9.00 \mathrm{E}-04$ & $6.00 \mathrm{E}-06$ & 9.9 & 0.001 & 2 & 392.4983 & $9.88 \mathrm{E}-04$ & 0.7407 & 2.7669 & converges in $300 \mathrm{~min}$ \\
\hline $9.00 \mathrm{E}-04$ & $7.00 \mathrm{E}-06$ & 9.9 & 0.001 & 2 & 666.8317 & $9.30 \mathrm{E}-04$ & 0.702 & 10.932 & converges in $600 \mathrm{~min}$ \\
\hline 4.00E-04 & $4.00 \mathrm{E}-06$ & 9.9 & 0.001 & 2 & 716.4983 & $9.98 \mathrm{E}-04$ & 0.4633 & 7.0095 & converges in $500 \mathrm{~min}$ \\
\hline $5.00 \mathrm{E}-04$ & $4.00 \mathrm{E}-06$ & 9.9 & 0.001 & 2 & 258.8317 & $8.50 \mathrm{E}-04$ & 0.766 & 4.9479 & converges in $175 \mathrm{~min}$ \\
\hline $6.00 \mathrm{E}-04$ & $4.00 \mathrm{E}-06$ & 9.9 & 0.001 & 2 & 302.4983 & $9.80 \mathrm{E}-04$ & 0.9661 & 1.8418 & converges in $250 \mathrm{~min}$ \\
\hline 7.00E-04 & $4.00 \mathrm{E}-06$ & 9.9 & 0.001 & 2 & 618.4983 & $9.17 E-04$ & 0.9011 & 4.5161 & converges in $425 \mathrm{~min}$ \\
\hline 8.00E-04 & 4.00E-06 & 9.9 & 0.001 & 2 & 197.165 & $5.74 \mathrm{E}-04$ & 0.7537 & 1.1883 & converges in $100 \mathrm{~min}$ \\
\hline $9.00 \mathrm{E}-04$ & $4.00 \mathrm{E}-06$ & 9.9 & 0.001 & 2 & 302.165 & $9.68 \mathrm{E}-04$ & 0.8217 & 2.9356 & converges in $250 \mathrm{~min}$ \\
\hline
\end{tabular}

Page 131 


\begin{tabular}{|c|c|c|c|c|c|c|c|c|c|}
\hline $1.00 \mathrm{E}-03$ & 4.00E-06 & 9.9 & 0.001 & 2 & 435.8317 & 9.99E-04 & 1.2235 & 4.3806 & converges in $400 \mathrm{~min}$ \\
\hline 2.00E-03 & 4.00E-06 & 9.9 & 0.001 & 2 & dnc & 0.6669 & dnc & 55.0658 & yaw instability $\sim 0.05$ \\
\hline 8.00E-04 & $3.00 \mathrm{E}-06$ & 9.9 & 0.001 & 2 & 616.165 & $1.00 \mathrm{E}-03$ & 0.5892 & 4.2469 & converges in $450 \mathrm{~min}$ \\
\hline 8.00E-04 & 4.00E-06 & 9.9 & 0.001 & 2 & 197.165 & 0.000574 & 0.7537 & 1.1883 & converges in $100 \mathrm{~min}$ \\
\hline 8.00E-04 & 5.00E-06 & 9.9 & 0.001 & 2 & 193.4983 & 8.43E-04 & 1.1333 & 1.0403 & converges in $125 \mathrm{~min}$ \\
\hline 8.00E-04 & $6.00 \mathrm{E}-06$ & 9.9 & 0.001 & 2 & 298.8317 & 8.99E-04 & 0.5819 & 1.2835 & converges in $150 \mathrm{~min}$ \\
\hline 8.00E-04 & 7.00E-06 & 9.9 & 0.001 & 2 & 426.165 & $8.12 \mathrm{E}-04$ & 0.8746 & 5.6192 & converges in $350 \mathrm{~min}$ \\
\hline $7.00 \mathrm{E}-04$ & 5.00E-06 & 9.9 & 0.001 & 2 & 255.4983 & $4.82 \mathrm{E}-04$ & 1.4353 & 2.8671 & converges in $200 \mathrm{~min}$ \\
\hline 8.00E-04 & 5.00E-06 & 9.9 & 0.001 & 2 & 197.165 & 0.000574 & 0.7537 & 1.1883 & converges in $100 \mathrm{~min}$ \\
\hline 9.00E-04 & 5.00E-06 & 9.9 & 0.001 & 2 & 617.4983 & 4.80E-04 & 1.0165 & 4.3888 & converges in $450 \mathrm{~min}$ \\
\hline
\end{tabular}

Page 132 
PD Controller Pulse Time Selection

Magnetorquers: Current Limiters Enabled not scaled by I_total

gyroscopic cancelation

\begin{tabular}{|c|c|c|c|c|}
\hline \multirow{4}{*}{$\begin{array}{l}\text { Rate Gain } \\
\text { C1 = } \\
d^{*} \text { eye(3) }\end{array}$} & \multicolumn{4}{|c|}{ Settings } \\
\hline & Orientation & & & \\
\hline & Gain & & norm(w_err) & pointing err \\
\hline & C2 = k*eye(3) & $\mathrm{dt}$ & $<x[\mathrm{deg} / \mathrm{s}]$ & $<x$ [deg] \\
\hline $8.00 \mathrm{E}-04$ & $5.00 \mathrm{E}-06$ & 16.9 & 0.001 & \\
\hline $8.00 \mathrm{E}-04$ & $5.00 \mathrm{E}-06$ & 15.9 & 0.001 & \\
\hline $8.00 \mathrm{E}-04$ & $5.00 \mathrm{E}-06$ & 14.9 & 0.001 & \\
\hline 8.00E-04 & $5.00 \mathrm{E}-06$ & 13.9 & 0.001 & \\
\hline 8.00E-04 & $5.00 \mathrm{E}-06$ & 12.9 & 0.001 & \\
\hline 8.00E-04 & $5.00 \mathrm{E}-06$ & 11.9 & 0.001 & \\
\hline $8.00 \mathrm{E}-04$ & $5.00 \mathrm{E}-06$ & 10.9 & 0.001 & \\
\hline 8.00E-04 & 5.00E-06 & 9.9 & 0.001 & \\
\hline 8.00E-04 & $5.00 \mathrm{E}-06$ & 8.9 & 0.001 & \\
\hline $8.00 \mathrm{E}-04$ & $5.00 \mathrm{E}-06$ & 7.9 & 0.001 & \\
\hline $8.00 \mathrm{E}-04$ & $5.00 \mathrm{E}-06$ & 6.9 & 0.001 & \\
\hline $8.00 \mathrm{E}-04$ & $5.00 \mathrm{E}-06$ & 5.9 & 0.001 & \\
\hline $8.00 \mathrm{E}-04$ & $5.00 \mathrm{E}-06$ & 4.9 & 0.001 & \\
\hline 8.00E-04 & $5.00 \mathrm{E}-06$ & 3.9 & 0.001 & \\
\hline 8.00E-04 & $5.00 \mathrm{E}-06$ & 2.9 & 0.001 & \\
\hline $8.00 \mathrm{E}-04$ & $5.00 \mathrm{E}-06$ & 1.9 & 0.001 & \\
\hline $8.00 \mathrm{E}-04$ & $5.00 \mathrm{E}-06$ & 0.9 & 0.001 & \\
\hline
\end{tabular}

\begin{tabular}{|c|c|c|c|}
\hline \multirow{2}{*}{\multicolumn{3}{|c|}{$\begin{array}{c}\text { PD Controller Parameter Tests } \\
\text { q0 }=\left[\begin{array}{llll}0.5 & 0.5 & 0.5 & 0.5\end{array}\right]\end{array}$}} & $I 1=0.0049$ \\
\hline & & & $12=0.1236$ \\
\hline \multicolumn{3}{|c|}{$w 0=\left[\begin{array}{lll}0.005 & -0.005 & 0.002\end{array}\right]$} & $13=0.1238$ \\
\hline \multicolumn{3}{|c|}{ Convergence } & \\
\hline & Pointing & Total & \\
\hline Rate & Error & Charge & \\
\hline$[\mathrm{deg} / \mathrm{s}]$ & [deg] & Draw [Ah] & Comment \\
\hline 0.0269 & dnc & 40.5502 & Local instabilities \\
\hline $6.76 \mathrm{E}-04$ & 1.4074 & 16.2061 & Local instabilities \\
\hline 7.44E-04 & 0.34 & 19.2183 & Local instabilities \\
\hline $9.96 \mathrm{E}-04$ & 0.7914 & 1.7226 & Local instabilities \\
\hline $8.05 \mathrm{E}-04$ & 0.8907 & 1.4057 & Converges in 225 \\
\hline $8.25 \mathrm{E}-04$ & 1.0054 & 1.2062 & Converges in 225 \\
\hline $9.73 \mathrm{E}-04$ & 1.2234 & 1.092 & Converges in 125 \\
\hline 8.43E-04 & 1.0445 & 1.0403 & Converges in 110 \\
\hline 8.03E-04 & 0.7222 & 1.0434 & Converges in 125 \\
\hline $9.99 \mathrm{E}-04$ & 0.4902 & 3.5477 & Converges in 325 \\
\hline $9.84 \mathrm{E}-04$ & 0.5178 & 1.0604 & Converges in 125 \\
\hline $9.85 \mathrm{E}-04$ & 0.3918 & 1.0687 & Converges in 126 \\
\hline $8.14 \mathrm{E}-04$ & 0.5141 & 1.1133 & Converges in 100 \\
\hline 7.90E-04 & 0.4805 & 1.1878 & Converges in 225 \\
\hline $9.99 \mathrm{E}-04$ & 0.5532 & 1.1083 & Converges in 125 \\
\hline $9.96 \mathrm{E}-04$ & 0.2502 & 1.1161 & Converges in 150 \\
\hline $976 \mathrm{E}$ & 0.4109 & 3 & Converge \\
\hline
\end{tabular}


PD Controller Quaternion Minimum Resolution

\begin{tabular}{|c|c|c|c|c|c|c|c|c|c|c|}
\hline \multicolumn{4}{|c|}{$\begin{array}{l}\text { Magnetorquers: Current Limiters Enabled } \\
\text { not scaled by I_total }\end{array}$} & $\begin{array}{r}\text { Param } \\
\mathrm{q} 0=[0.5 \\
\mathrm{w} 0=[0 \\
0\end{array}$ & $\begin{array}{l}\text { ter Tests } \\
.50 .50 .5] \\
05-0.005 \\
02 \text { ] }\end{array}$ & & $\begin{array}{l}11=0.0049 \\
12=0.1236\end{array}$ & & $\begin{array}{l}10 \text { orbits } \\
475 \mathrm{~km}\end{array}$ & \\
\hline Settings & & & & & & Convergenc & & & & \\
\hline $\begin{array}{l}\text { Rate Gain } \\
\text { C1 } \\
\text { C1 = } \\
d^{*} \text { eye }(3)\end{array}$ & $\begin{array}{l}\text { Orientation } \\
\text { Gain C2 } \\
\mathrm{C} 2= \\
\mathrm{k}^{*} \text { eye(3) }\end{array}$ & $\mathrm{dt}$ & $\begin{array}{c}\text { norm(w_err) } \\
<x[\text { deg } / \mathrm{s}]\end{array}$ & $\begin{array}{l}\text { pointing } \\
\quad \text { err } \\
<x[\text { deg] }\end{array}$ & $\begin{array}{l}\text { Quaternion } \\
\text { Resolution }\end{array}$ & $\begin{array}{l}\text { Time to } \\
\text { Converge } \\
\text { [min] }\end{array}$ & $\begin{array}{c}\text { Rate } \\
{[\mathrm{deg} / \mathrm{s}]}\end{array}$ & $\begin{array}{l}\text { Pointing } \\
\text { Error } \\
\text { [deg] }\end{array}$ & $\begin{array}{c}\text { Total } \\
\text { Charge } \\
\text { Draw } \\
\text { [Ah] }\end{array}$ & Comment \\
\hline 8.00E-04 & 5.00E-06 & 9.9 & 0.001 & 2 & $1.00 \mathrm{E}-09$ & 193.4983 & $8.43 \mathrm{E}-04$ & 1.0445 & 1.0403 & \\
\hline 8.00E-04 & $5.00 \mathrm{E}-06$ & 9.9 & 0.001 & 2 & 1.00E-08 & 193.4983 & 8.43E-04 & 1.0445 & 1.0403 & \\
\hline $8.00 \mathrm{E}-04$ & 5.00E-06 & 9.9 & 0.001 & 2 & $1.00 \mathrm{E}-07$ & 193.4983 & 8.43E-04 & 1.0445 & 1.0403 & \\
\hline $8.00 \mathrm{E}-04$ & 5.00E-06 & 9.9 & 0.001 & 2 & 1.00E-06 & 193.4983 & 8.43E-04 & 1.0445 & 1.0403 & \\
\hline 8.00E-04 & 5.00E-06 & 9.9 & 0.001 & 2 & 1.00E-05 & 193.4983 & 8.43E-04 & 1.0445 & 1.0403 & \\
\hline $8.00 \mathrm{E}-04$ & $5.00 \mathrm{E}-06$ & 9.9 & 0.001 & 2 & 1.00E-04 & 192.4983 & $9.00 \mathrm{E}-04$ & 1.1285 & 1.0389 & \\
\hline $8.00 \mathrm{E}-04$ & $5.00 \mathrm{E}-06$ & 9.9 & 0.001 & 2 & $1.00 \mathrm{E}-03$ & 216.4983 & $5.95 \mathrm{E}-04$ & 0.8731 & 1.0379 & \\
\hline 8.00E-04 & $5.00 \mathrm{E}-06$ & 9.9 & 0.001 & 2 & $5.00 \mathrm{E}-03$ & 289.4983 & $9.80 \mathrm{E}-04$ & 0.2933 & 1.0592 & \\
\hline $8.00 \mathrm{E}-04$ & 5.00E-06 & 9.9 & 0.001 & 2 & $1.00 \mathrm{E}-02$ & 255.8317 & $7.22 \mathrm{E}-04$ & 1.2605 & 1.0548 & $\begin{array}{l}\text { Rounding errors exceed } 10 \\
\text { deg } \\
\text { Rounding errors exceed } 30\end{array}$ \\
\hline $8.00 \mathrm{E}-04$ & 5.00E-06 & 9.9 & 0.001 & 2 & $5.00 \mathrm{E}-02$ & 301.8317 & $2.83 \mathrm{E}-04$ & 0.8452 & 1.2456 & $\begin{array}{l}\text { deg } \\
\text { Rounding errors exceed } 40\end{array}$ \\
\hline $8.00 \mathrm{E}-04$ & $5.00 \mathrm{E}-06$ & 9.9 & 0.001 & 2 & $1.00 \mathrm{E}-01$ & 582.4983 & $6.70 \mathrm{E}-04$ & 0.5558 & 1.8512 & deg \\
\hline $8.00 \mathrm{E}-04$ & $5.00 \mathrm{E}-06$ & 9.9 & 0.001 & 2 & 5.00E-01 & . & & & & \\
\hline
\end{tabular}


PD Controller Quaternion Maximum Noise

Magnetorquers: Current Limiters Enabled

$$
\text { not scaled by I_total }
$$

gyroscopic cancelation

\begin{tabular}{|c|c|c|c|}
\hline \multicolumn{4}{|l|}{ Settings } \\
\hline Rate & Orientation & & \\
\hline $\mathrm{C} 1=$ & $\mathrm{C} 2=$ & & norm(w_err) \\
\hline d*eye(3) & k*eye(3) & $d t$ & $<\mathrm{x}[\mathrm{deg} / \mathrm{s}]$ \\
\hline 8.00E-04 & 5.00E-06 & 9.9 & 0.001 \\
\hline 8.00E-04 & 5.00E-06 & 9.9 & 0.001 \\
\hline 8.00E-04 & 5.00E-06 & 9.9 & 0.001 \\
\hline 8.00E-04 & 5.00E-06 & 9.9 & 0.001 \\
\hline 8.00E-04 & 5.00E-06 & 9.9 & 0.001 \\
\hline 8.00E-04 & $5.00 \mathrm{E}-06$ & 9.9 & 0.001 \\
\hline 8.00E-04 & $5.00 \mathrm{E}-06$ & 9.9 & 0.001 \\
\hline 8.00E-04 & $5.00 \mathrm{E}-06$ & 9.9 & 0.001 \\
\hline 8.00E-04 & $5.00 \mathrm{E}-06$ & 9.9 & 0.001 \\
\hline 8.00E-04 & $5.00 \mathrm{E}-06$ & 9.9 & 0.001 \\
\hline 8.00E-04 & $5.00 \mathrm{E}-06$ & 9.9 & 0.001 \\
\hline
\end{tabular}

\section{PD Controller \\ Parameter Tests \\ q0 $=\left[\begin{array}{llll}0.5 & 0.5 & 0.5 & 0.5\end{array}\right]$ \\ w0 $=[0.005-0.005$ \\ 0.002]}

\section{pointing Quaternion}

$<\mathrm{x}$ [deg] std dev

std_dev

1.00E-09

1.00E-08

$1.00 \mathrm{E}-07$

$1.00 \mathrm{E}-06$

2 1.00E-0

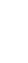

2

$\begin{array}{lllll}2 & 5.00 \mathrm{E}-03 & 282.4983 & 9.64 \mathrm{E}-04 & 0.6745 \\ & & & & \end{array}$

$\begin{array}{lllll}2 & 1.00 \mathrm{E}-02 & 151.8317 & 9.59 \mathrm{E}-04 & 0.6124 \\ 2 & 5.00 \mathrm{E}-02 & 769.8317 & 9.69 \mathrm{E}-04 & 2.7363\end{array}$

$\begin{array}{lllll}2 & 5.00 \mathrm{E}-02 & 769.8317 & 9.69 \mathrm{E}-04 & 2.7363 \\ 2 & 1.00 \mathrm{E}-01 & \mathrm{dnc} & 1.17 \mathrm{E}-01 & \mathrm{dnc}\end{array}$

10 orbits

$475 \mathrm{~km}$

incl $=95$

deg

Convergence

Time to Pointing Total

Rate

[deg/s] [deg] Draw [Ah]

$\begin{array}{llll} & 8.43 \mathrm{E}-04 & 1.0445 & 1.0403\end{array}$

$\begin{array}{llll}193.4983 & 8.43 \mathrm{E}-04 & 1.0445 & 1.0403\end{array}$

$193.4983 \quad 8.43 \mathrm{E}-04 \quad 1.0445 \quad 1.0403$

$\begin{array}{llll}193.4983 & 8.43 \mathrm{E}-04 & 1.0445 & 1.0403\end{array}$

$\begin{array}{llll}93.4983 & 8.38 \mathrm{E}-04 & 1.0424 & 1.0405\end{array}$

noise $\sim 2$

$1.046 \mathrm{deg}$

noise $\sim 8$

$1.0423 \mathrm{deg}$

noise $\sim 15$

1.1041 deg

noise 21

$1.0891 \mathrm{deg}$

noise $\sim 60$

3.4052 deg

12.1235 
PD Controller Rate Minimum Resolution

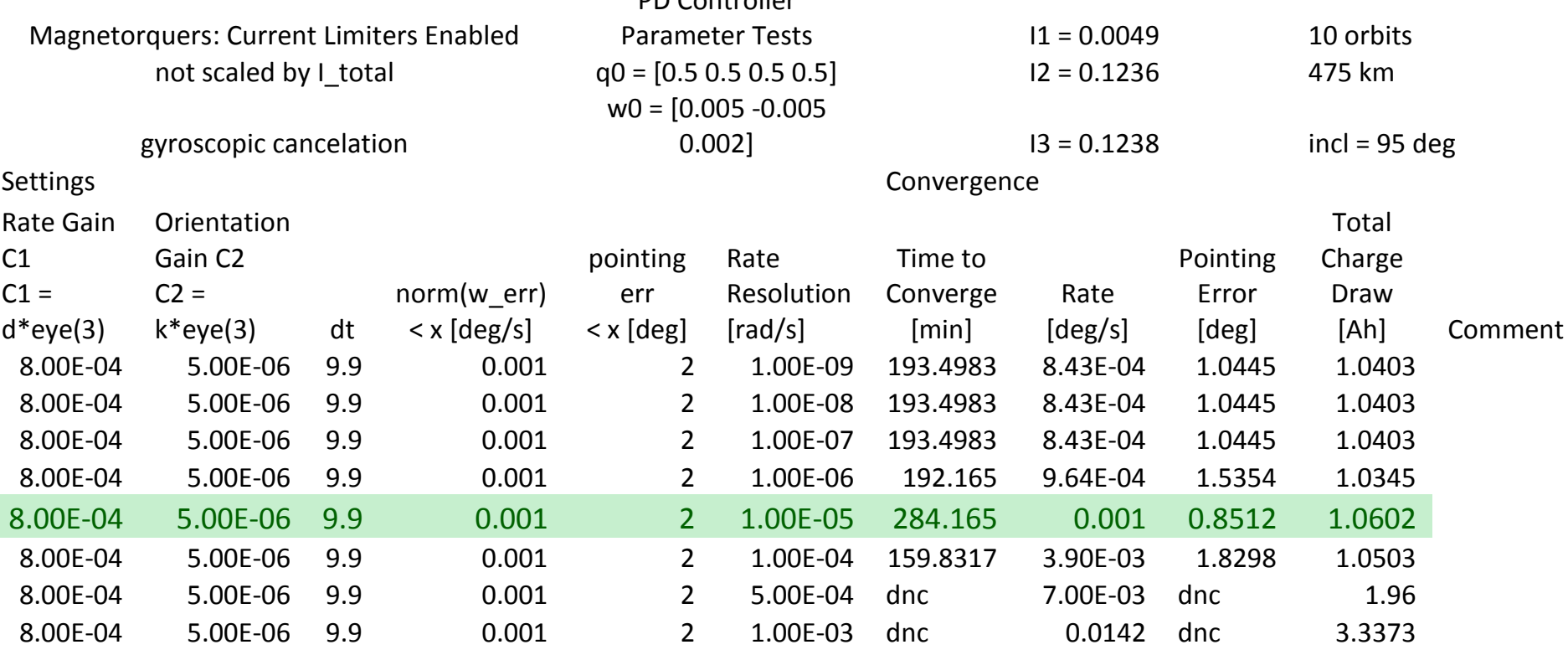


PD Controller Rate Maximum Noise

\begin{tabular}{|c|c|c|c|c|c|c|c|c|c|c|}
\hline \multirow{2}{*}{\multicolumn{4}{|c|}{ Magnetorquers: Current Limiters Enabled }} & & \multirow{2}{*}{\multicolumn{3}{|c|}{$11=0.0049$}} & \multirow{2}{*}{\multicolumn{2}{|c|}{10 orbits }} \\
\hline & & & & \multirow{3}{*}{\multicolumn{2}{|c|}{ 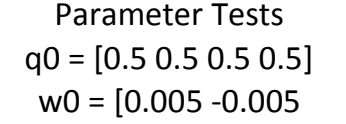 }} & & & & & \\
\hline & \multirow{2}{*}{\multicolumn{3}{|c|}{ not scaled by I_total }} & & & & \multirow{2}{*}{\multicolumn{2}{|c|}{$12=0.1236$}} & \multirow{2}{*}{\multicolumn{2}{|c|}{$475 \mathrm{~km}$}} \\
\hline & & & & & & & & & & \\
\hline & \multicolumn{3}{|c|}{ gyroscopic cancelation } & & 02] & & $13=0.1238$ & & incl $=95 \mathrm{de}$ & \\
\hline ttings & & & & & & \multicolumn{3}{|c|}{ Convergence } & & \\
\hline te Gain & Orientation & & & & & & & & Total & \\
\hline & Gain C2 & & & pointing & & Time to & & Pointing & Charge & \\
\hline$=$ & $\mathrm{C} 2=$ & & norm(w_err) & err & Rate noise & Converge & Rate & Error & Draw & \\
\hline *eye(3) & $k^{*}$ eye(3) & $\mathrm{dt}$ & $<x[\operatorname{deg} / \mathrm{s}]$ & $<x$ [deg] & {$[\mathrm{rad} / \mathrm{s}]$} & [min] & [deg/s] & [deg] & [Ah] & Comment \\
\hline 8.00E-04 & $5.00 \mathrm{E}-06$ & 9.9 & 0.001 & 2 & $1.00 \mathrm{E}-09$ & 193.4983 & $8.43 \mathrm{E}-04$ & 1.0445 & 1.0403 & \\
\hline 8.00E-04 & $5.00 \mathrm{E}-06$ & 9.9 & 0.001 & 2 & $1.00 \mathrm{E}-08$ & 193.4983 & $8.43 \mathrm{E}-04$ & 1.0445 & 1.0403 & \\
\hline 8.00E-04 & 5.00E-06 & 9.9 & 0.001 & 2 & $1.00 \mathrm{E}-07$ & 256.4983 & $3.65 \mathrm{E}-04$ & 0.9758 & 1.0428 & \\
\hline 8.00E-04 & $5.00 \mathrm{E}-06$ & 9.9 & 0.001 & 2 & $1.00 \mathrm{E}-06$ & 255.165 & $5.52 \mathrm{E}-04$ & 0.572 & 1.0436 & \\
\hline 8.00E-04 & 5.00E-06 & 9.9 & 0.001 & 2 & 1.00E-05 & 241.165 & 9.56E-04 & 0.834 & 1.0459 & \\
\hline 8.00E-04 & $5.00 \mathrm{E}-06$ & 9.9 & 0.001 & 2 & $1.00 \mathrm{E}-04$ & 247.8317 & $9.30 \mathrm{E}-03$ & 1.0238 & 1.1885 & \\
\hline 8.00E-04 & $5.00 \mathrm{E}-06$ & 9.9 & 0.001 & 2 & $5.00 \mathrm{E}-04$ & dnc & $5.32 \mathrm{E}-02$ & dnc & 5.9397 & \\
\hline
\end{tabular}




\section{Wheel Spin Up Simulations}

Wheel spinup

\begin{tabular}{|c|c|c|c|c|c|c|c|c|c|c|c|c|c|}
\hline \multicolumn{8}{|c|}{ Settings } & \multicolumn{6}{|c|}{ Convergence } \\
\hline & & & & & & & & Time for & & & & & \\
\hline Rate & Orientation & & norm & pointing & Torque & & & Wheel to & & & & Total & \\
\hline Gain C1 & Gain C2 & & (w_err) & err & Control & Wheel & & reach & Time to & & Pointing & Current & \\
\hline $\begin{array}{l}c 1= \\
d^{*} \text { eye }(3)\end{array}$ & $\begin{array}{l}c 2= \\
k^{*} \text { eye }(3)\end{array}$ & $\mathrm{dt}$ & $\begin{array}{c}<x \\
{[\operatorname{deg} / \mathrm{s}]}\end{array}$ & $\begin{array}{c}<x \\
{[\operatorname{deg}]}\end{array}$ & $\begin{array}{l}\text { Noise } \\
{[\mathrm{Nm}]}\end{array}$ & $\begin{array}{l}\text { Speed } \\
\text { [RPM] }\end{array}$ & Tw_desired & $\begin{array}{l}\text { speed } \\
\text { [min] }\end{array}$ & $\begin{array}{l}\text { Converge } \\
\text { [min] }\end{array}$ & $\begin{array}{c}\text { Rate } \\
{[\mathrm{deg} / \mathrm{s}]}\end{array}$ & $\begin{array}{l}\text { Error } \\
\text { [deg] }\end{array}$ & $\begin{array}{c}\text { Draw } \\
{[\mathrm{A}]}\end{array}$ & Comment \\
\hline $8.00 E-04$ & 5.00E-06 & 9.9 & 0.001 & 2 & 0 & 2000 & $1.00 \mathrm{E}-07$ & 977.8333 & dnc & 0.4418 & dnc & 20.2599 & $\begin{array}{l}4 \text { deg pitch offset, } \\
\text { anomaly @ } 450 \text { min }\end{array}$ \\
\hline $8.00 \mathrm{E}-04$ & 5.00E-06 & 9.9 & 0.001 & 2 & 0 & 2000 & 2.00E-07 & 488.8333 & 504.165 & $7.65 E-04$ & 1.5399 & 1.4356 & 7 deg pitch offset \\
\hline $8.00 \mathrm{E}-04$ & 5.00E-06 & 9.9 & 0.001 & 2 & 0 & 2000 & 3.00E-07 & 326.1667 & 360.8317 & $8.28 \mathrm{E}-04$ & 1.9023 & 5.0168 & $\begin{array}{l}10 \text { deg pitch offset, } \\
\text { anomaly @ } 200 \text { min }\end{array}$ \\
\hline $8.00 \mathrm{E}-04$ & 5.00E-06 & 9.9 & 0.001 & 2 & 0 & 2000 & 4.00E-07 & 244.5 & dnc & 0.0075 & dnc & 4.3374 & $\begin{array}{l}14 \text { deg pitch offset, } \\
\text { anomaly @ } 150 \text { min }\end{array}$ \\
\hline $8.00 \mathrm{E}-04$ & 5.00E-06 & 9.9 & 0.001 & 2 & 0 & 2000 & $5.00 \mathrm{E}-07$ & 195.8333 & 227.165 & 7.27E-04 & 1.0641 & 2.4467 & $\begin{array}{l}18 \text { deg pitch offset, } \\
\text { anomaly @ } 100 \text { min }\end{array}$ \\
\hline $5.00 \mathrm{E}-04$ & 5.00E-06 & 9.9 & 0.001 & 2 & 0 & 2000 & $5.00 \mathrm{E}-07$ & 195.8333 & 239.4983 & $1.57 E-04$ & 1.2646 & 1.3045 & 18 deg pitch offset \\
\hline $5.00 \mathrm{E}-04$ & 5.00E-06 & 9.9 & 0.001 & 2 & $1.00 \mathrm{E}-06$ & 2000 & $5.00 \mathrm{E}-07$ & 195.8333 & 239.4983 & 3.31E-04 & 1.4316 & 1.3169 & $\begin{array}{l}15-20 \text { deg pitch } \\
\text { offset }\end{array}$ \\
\hline 5.00E- & & & & & & & & & & & & & \\
\hline 04 & 5.00E-06 & 9.9 & 0.001 & 2 & 0 & 2000 & 2.00E-07 & 488.8333 & 518.165 & $5.45 \mathrm{E}-04$ & 1.1792 & 1.2411 & 7 deg pitch offset \\
\hline $\begin{array}{r}5.00 \mathrm{E}- \\
04\end{array}$ & $5.00 \mathrm{E}-06$ & 9.9 & 0.001 & 2 & $\begin{array}{r}1.00 \mathrm{E}- \\
06\end{array}$ & 2000 & $2.00 \mathrm{E}-07$ & 488.8333 & 531.8317 & 5.37E-04 & 1.2555 & 1.3122 & $\begin{array}{l}\text { 3-9 deg pitch } \\
\text { offset }\end{array}$ \\
\hline
\end{tabular}

